NBSIR 88-3716

\title{
Proceedings of the Symposium on Gaseous and Vaporous Removal Equipment Test Methods
}

Held at the National Bureau of Standards

September 2, 1986

Preston E. McNall, Editor

U.S. DEPARTMENT OF COMMERCE

National Bureau of Standards

National Engineering Laboratory

Center for Building Technology

Building Environment Division

Gaithersburg, MD 20899

February 1988

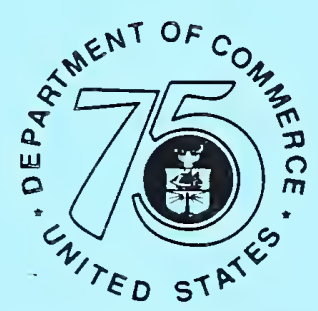

Stimulating America's Progress 1913-1988

Sponsored by:

U.S. DEPARTMENT OF COMMERCE

National Bureau of Standards

Gaithersburg, MD 20899 

PROCEEDINGS OF THE SYMPOSIUM ON GASEOUS AND VAPOROUS REMOVAL EQUIPMENT TEST METHODS

Held at the National Bureau of Standards

September 2, 1986

Preston E. McNall, Editor

U.S. DEPARTMENT OF COMMERCE

National Bureau of Standards

National Engineering Laboratory

Center for Building Technology

Building Environment Division

Gaithersburg, MD 20899

February 1988

Sponsored by:

U.S. DEPARTMENT OF COMMERCE

National Bureau of Standards

Gaithersburg, MD 20899

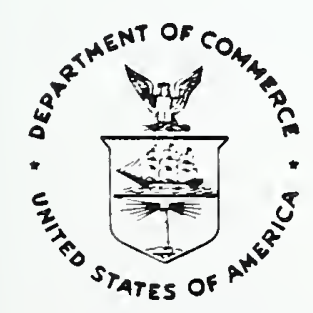

U.S. DEPARTMENT OF COMMERCE, C. William Verity, Secretary

NATIONAL BUREAU OF STANDARDS, Ernest Ambler, Director 
•

. 
This symposium proceedings summarizes the current state-of-the-art on gaseous and vaporous removal test methods for equipment designed for use in the general ventilation of buildings. Papers by the ten (10) invited authors are included. A discussion section outlines the conclusions reached concerning the future direction for test method development.

Key Words: absorption, adsorption, air cleaning, catalysis, chemisorption, gaseous, validation and vapors. 
Introduction by Preston E. McNall $\ldots \ldots \ldots \ldots \ldots \ldots \ldots \ldots \ldots \ldots \ldots \ldots \ldots$

Acknowledgement $\quad \ldots \ldots \ldots \ldots \ldots \ldots \ldots \ldots \ldots \ldots \ldots \ldots \ldots \ldots \ldots \ldots \ldots \ldots \ldots \ldots \ldots$

Partial Glossary of Terms $\ldots \ldots \ldots \ldots \ldots \ldots \ldots \ldots \ldots \ldots \ldots \ldots \ldots \ldots \ldots \ldots$

Activated Charcoal Performance in Removing Toxic Waste by

Victor R. Deitz .................................. 4

Optimizing Carbon for Removal of Certain Contaminants by Amos Turk .. 12

Reduction in Gaseous Contaminant Concentration with the Use of In-Duct Complex Electrical Fields by Allan H. Frey ........... 15

The Control of Indoor Air Quality - The Use of Impregnated Alumina

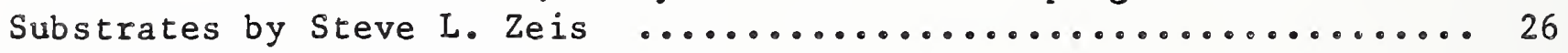

Monitoring Mass Transfer Zones in Adsorbers by Basil G. Louros ..... 28

Characterization of the LTC Catalyst: Performance Against Common

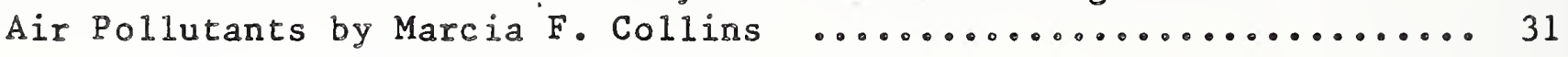

Effects of Relative Humidity on Adsorption of Contaminants on Activated Carbon by Dwight W. Underhill, Gina Micarelli and Maria Javorsky .................................. 42

Practical Test Requirements for Gaseous Contaminant Removal Equipment

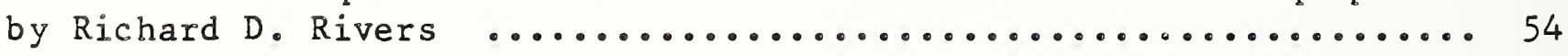

Comparative Performance for Gas Removal by Cyrus M. Bosworth ...... 65

Comparison of Chromotropic Acid, Pararosaniline, and Gravimetric Formaldehyde Determinations by Samuel Silberstein ............ 73

Discussion and Conclusions by Preston E. McNall .............. 83 


\section{INTRODUCTION}

Preston E. McNall

National Bureau of Standards

For many years, gaseous and vaporous removal equipment has been used in general ventilation systems in buildings. The American Society of Heating, Refrigerating and Air-Conditioning Engineers (ASHRAE), the American Society for Testing Materials (ASTM), and others have considered test standards for such apparatus without notable success. As a result, we have used standard methods to evaluate particulate filtering systems, but no standard methods exist for gaseous and vaporous removal equipment for use in general ventilation systems. Over the last decade, the energy crisis has focused attention on the energy cost of building ventilation, and increased research has also brought attention to many gaseous and vaporous pollutants which can be present in inside air.

These factors have intensified the need for standard evaluation methods for such equipment, as their application is increasing, and many new devices are being introduced into the marketplace.

This symposium was organized to bring together a number of experts in the field to examine the state of the art and summarize, insofar as possible, the conclusions concerning the future of test methods and provide a frameowrk for their development and/or future necessary research.

The problem seems complex. There is a wide variety of materials with differing physical and chemical characteristics of interest. A wide range of concentration levels of various pollutants are found in indoor air. Several known removal or mitigation methods have been employed, such as adsorption, absorption, chemisorption, catalysis, and complex electrical fields.

The symposium proceedings are presented as an aid to those who may contribute to the growing need for standardized test methods which will be useful for orderly commerce among the suppliers and users of such devices. 
Acknowledgment

The participation of all those who presented information in this symposium is gratefully appreciated. 


\section{Glossary of Terms}

Some definitions are listed to assist readers who are not thoroughly familiar with this field.

Absorption - A substance dissolved in another, such as oxygen dissolved in water.

Adsorption - A substance retained on the surface of another, such as carbon tetrachloride adsorbed on activated carbon.

Breakthrough - A condition which exists with a sorption device, when a specified penetration of a challenge contaminant is reached. (The massloading at which breakthrough occurs is dependent on many parameters, such as bed depth, velocity, temperature, humidity, challenge concentration, etc.).

Capacity - The ability of a sorption device to retain a material before breakthrough.

Catalyst - A material which accelerates a chemical reaction, whose concentration is unchanged.

Challenge - The concentration of a known material fed to an air treatment device.

Chemisorption - A substance taken up and held during the sorption process, usually irreversibly, in another material, by chemical reaction.

Efficiency - The ratio of concentration of a fed material less the outlet concentration divided by the fed concentration expressed as a percent.

$$
\text { eff }=\frac{\text { Cf }- \text { CO }}{\text { Cf }}
$$

Loading - The amount of a challenge material held by an air treatment device, at a specified condition.

Penetration - The ratio of the outlet concentration to the fed concentration of a material, expressed as a percent.

$$
P=\frac{C 0}{C f} \times 100=100 \text { eff }
$$




\author{
Victor R. Deitz \\ Naval Research Laboratory \\ Code 6170 \\ Washington, DC 20375-5000
}

\title{
1. Abstract
}

The applications of granular activated carbons for the removal of undesirable gases and vapors have been extended by their use as catalyst and impregnation supports. For long service times in ventilating systems, the atmospheric contaminants become important factors and can limit service efficiency. The moisture content of the air also is an important parameter. Carbons used with physically adsorbed gases can frequently be regenerated by extended air flows, but chemically adsorbed gases require special treatment and generally cannot be economically regenerated in situ. A test procedure for used carbon should reveal the residual $l$ ife as we 11 as the existing penetration of the test gas. Test procedures for new adsorbents are of value for procurement requirements, but need to be modified when used materials are examined. The relative humidity and the dew point of the air stream are both factors in a test procedure and the applications.

\section{Background Considerations}

The large volume of air processed in the ventilating systems of buildings contains significant amounts of atmospheric contaminants. Those that degrade an activated charcoal adsorbent ( $T a b l e ~ I)$ include ozone, sulfur dioxide, nitrogen oxides and the volatile hydrocarbons (non-methane). Although the concentrations are in the ppm range, the summation over extended service times degrades the efficiency of the charcoal. The example in Table 1 is a 30,000 CFM filter operating for 90 days in the air purification facility of a nuclear power plant. The reactive gases are ozone, sulfur dioxide and the nitrogen oxides.

Table 1: Atmospheric Contaminants Entering an Activated Charcoal Adsorber in 90 Days

\begin{tabular}{lccc} 
& PPm $\left(\mathrm{V} / \mathrm{V} \times 10^{-6}\right) *$ & Weight $(1 \mathrm{bs})$ & $\begin{array}{l}\text { wt } \\
\text { Charcoal }\end{array}$ \\
\hline ozone & 0.019 & 10 & 0.27 \\
$\mathrm{SO}_{2}$ & 0.023 & 16 & 0.43 \\
Nox & 0.043 & 21 & 0.56 \\
Hydrocarbons & 0.23 & 210 & 5.0
\end{tabular}

3-year average at NRL

The second factor that influences the efficiency of a charcoal adsorbent is the concentration of water vapor in the air flow. There are seasonal variations (Figure 1) in the monthly averages, which were calculated from observations over a 4-year period recorded at the National Airport. These results demonstrate that wet air is prevalent for June, July and August in the Washington area. The influence of the wet air on the adsorptive 


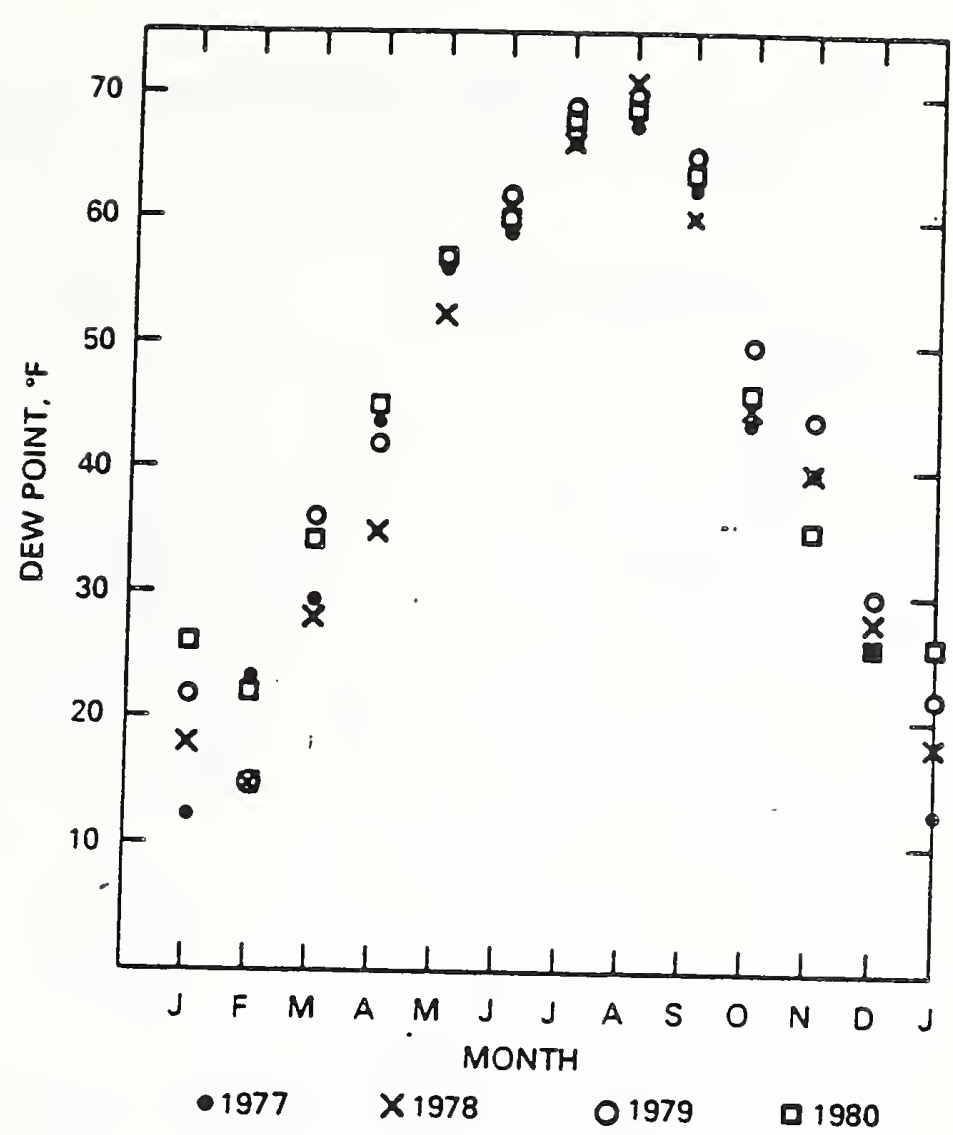

Figure 1: Seasonal variation of the monthly average dew point at the National Airport Weather Station, Washington, D.C.

properties of a whetlerite charcoal (Figure 2). shows a corresponding behavior for the adsorptive properties of samples exposed during one-month periods. In general, a high concentration of water vapor degrades the adsorption efficiency for a toxic vapor which in some cases can be recovered on exposure to a flow of dry air.

The breakthrough concentrations for a charcoal bed for toxic and obnoxious vapors occur at low surface coverage. The breakthrough will occur in shorter intervals as the time in service of the charcoal bed is extended. Both physical adsorption and chemisorption processes can take place simultaneously at different parts of an adsorbent surface.

\section{Removal via Physical Ad sorption}

Many organic vapors in air flows may be removed by charcoal adsorbents by a physical adsorption process. The extent to which this is accomplished depends on the carbon. An example (Figure 3) is the adsorption of benzene vapor at $30^{\circ} \mathrm{C}$ by each of four commercial activated carbons which differ in adsorptive surface area. When the amount adsorbed is divided by the monolayer coverage for each type of activated carbon, the four isotherms coincide, see Figure 4.

The water content of the air flow has a strong influence on the adsorption of benzene vapor. Adsorption is also dependent on the initial water content of the carbon. The results of J.K. Thompson (Figure 5) were obtained for air flows of different relative humidities and for carbons prehumidified at specified relative humidity. The points on the line of 


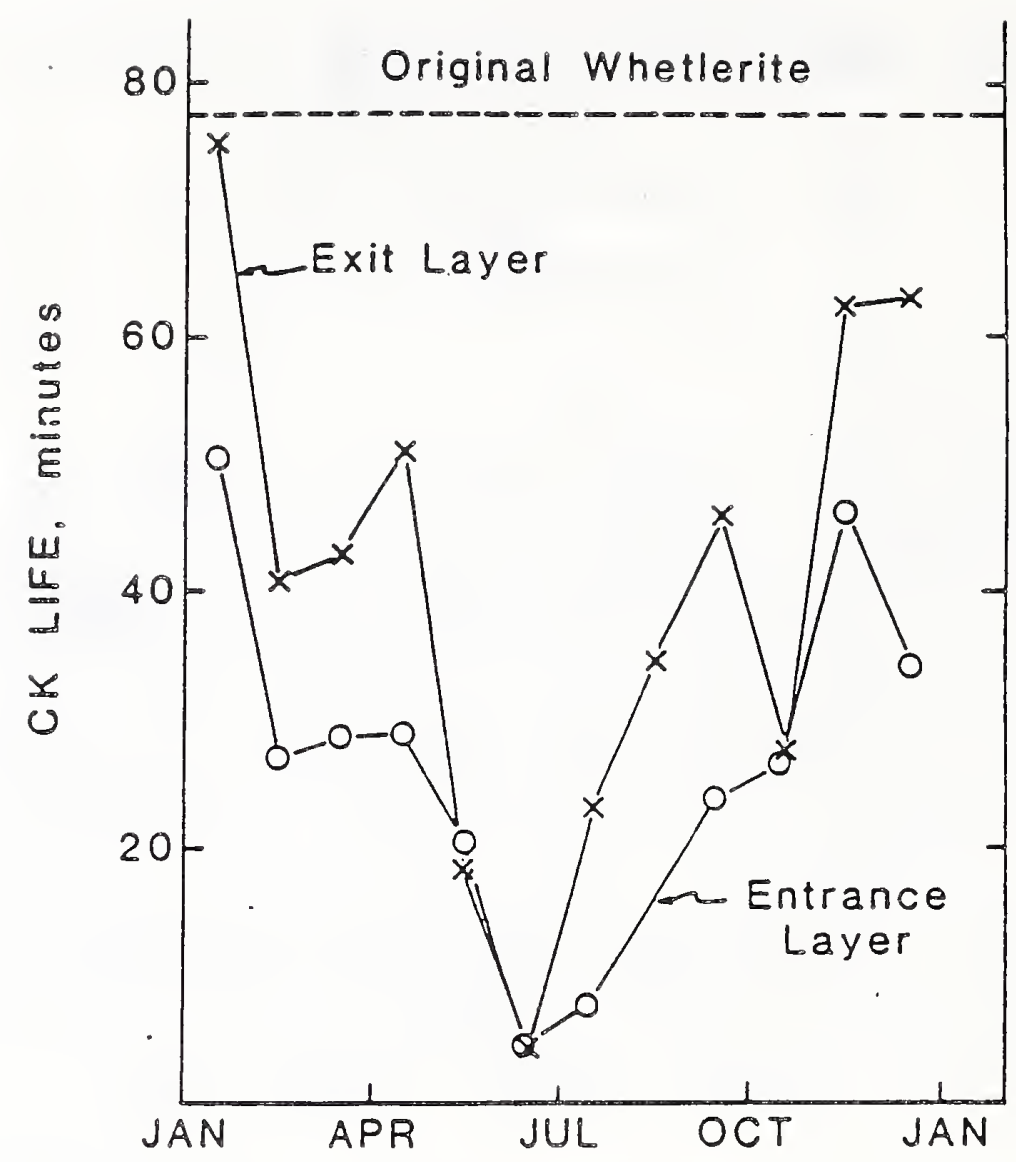

Figure 2: Seasonal influence on the removal of cyanogen chloride after weathering whetlerite in one-month intervals at NRL.

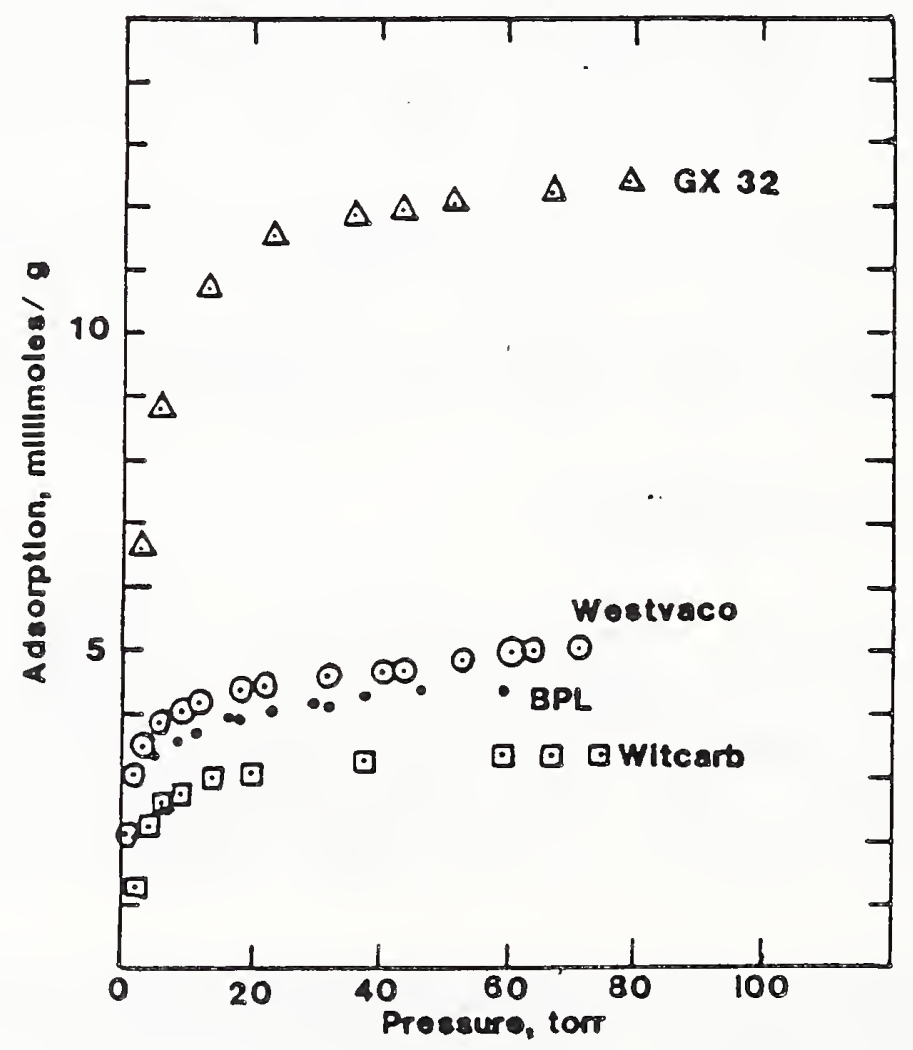

Figure 3: Adsorption isotherms (30\%) of benzene vapor on four coal-base charcoals. 


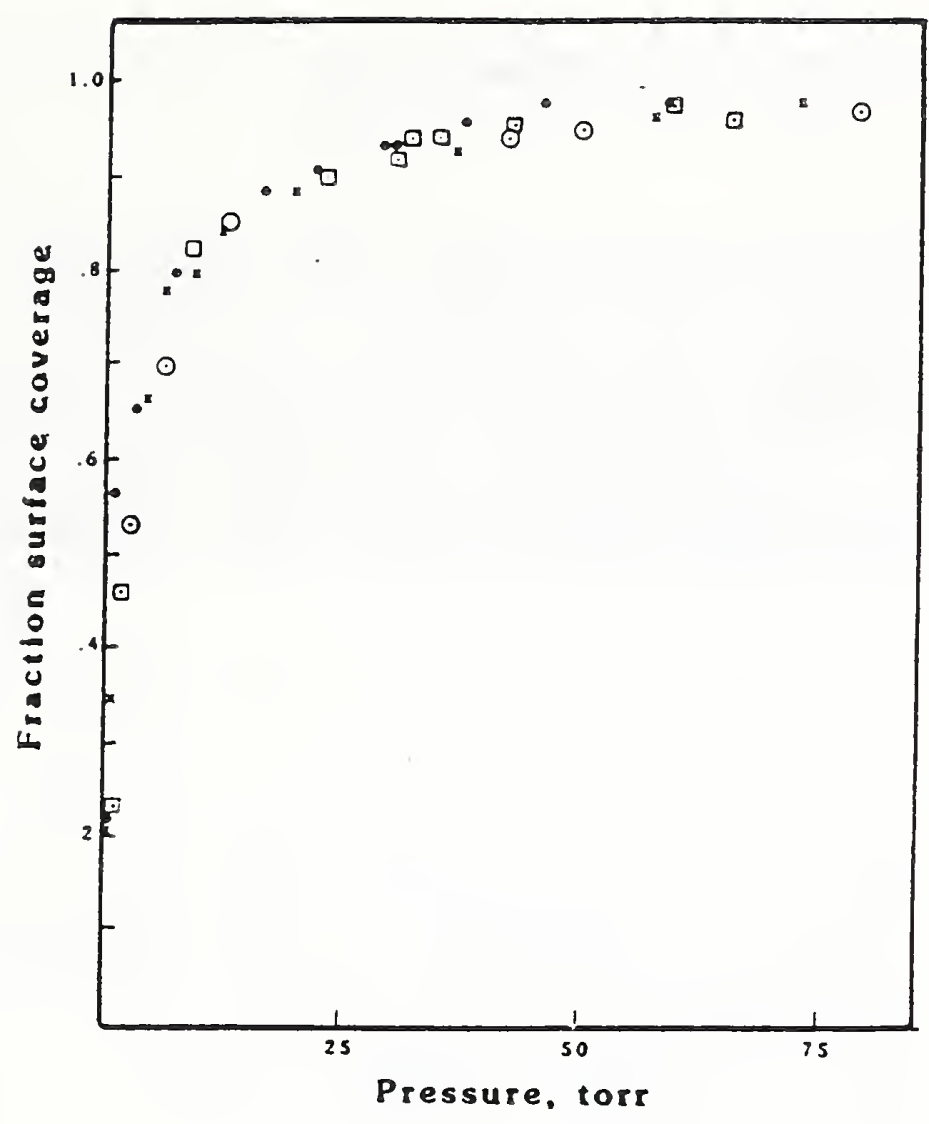

Figure 4: Benzene isotherms $\left(30^{\circ} \mathrm{C}\right)$ of Figure 3 plotted as fractional surface coverage.

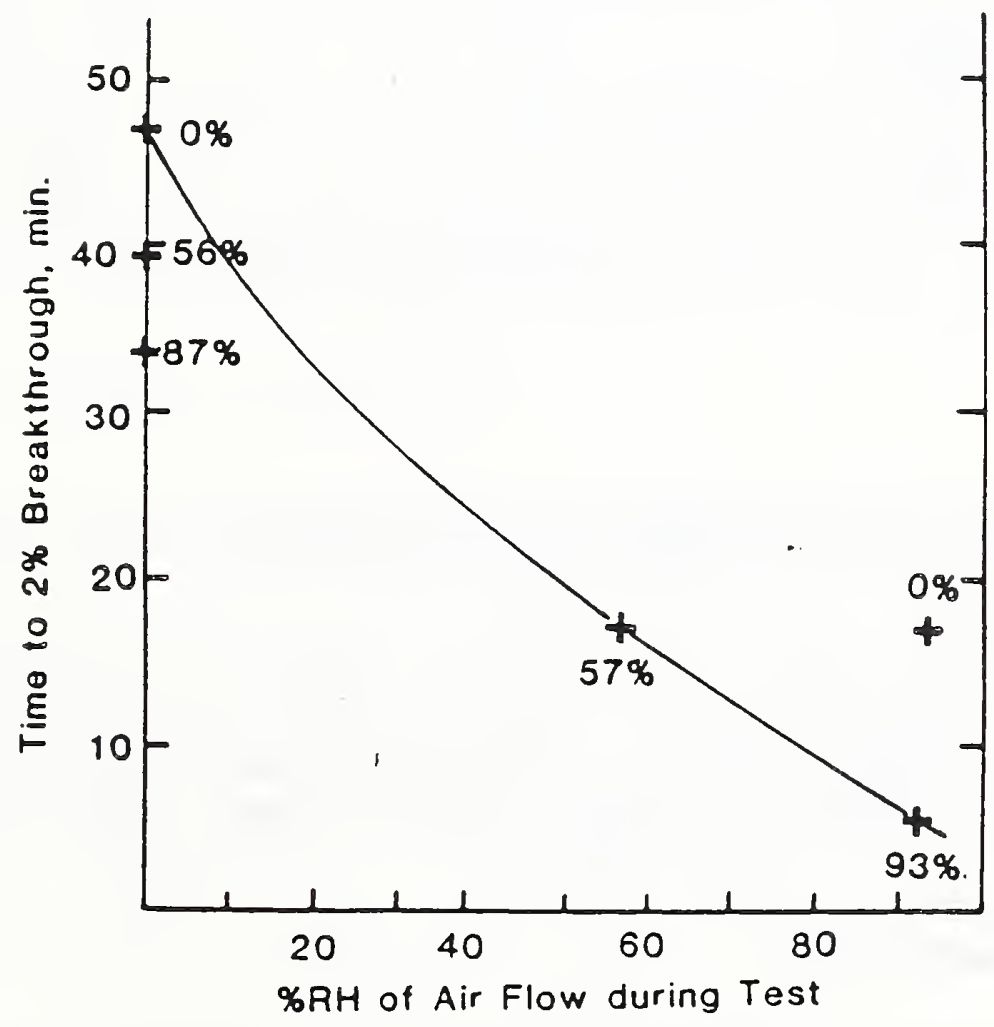

Figure 5: Influence of relative humidity on the adsorption of benzene vapor in air flows - measurements by J.R. Thompson, NRL 
Figure 5 were obtained when the prehumidified carbon (16 hours) was exposed to the same relative humidity as the air flow.

An important characteristic property of physical adsorption in a flowsystem is the continued penetration of the vapor after the challenge pollutant has been turned off. An example is the benzenemair system shown in Figure 6. The dose was $500 \mathrm{ppm}$ benzene in air and this was continued for 120 minutes; thereafter, only the air purge was continued at the same flow for 240 minutes. The effluent benzene gradual ly leveled-off and eventually decreased to the baseline (not shown). The behavior resembles the effluent from a chromatographic column under inefficieat separation conditions.

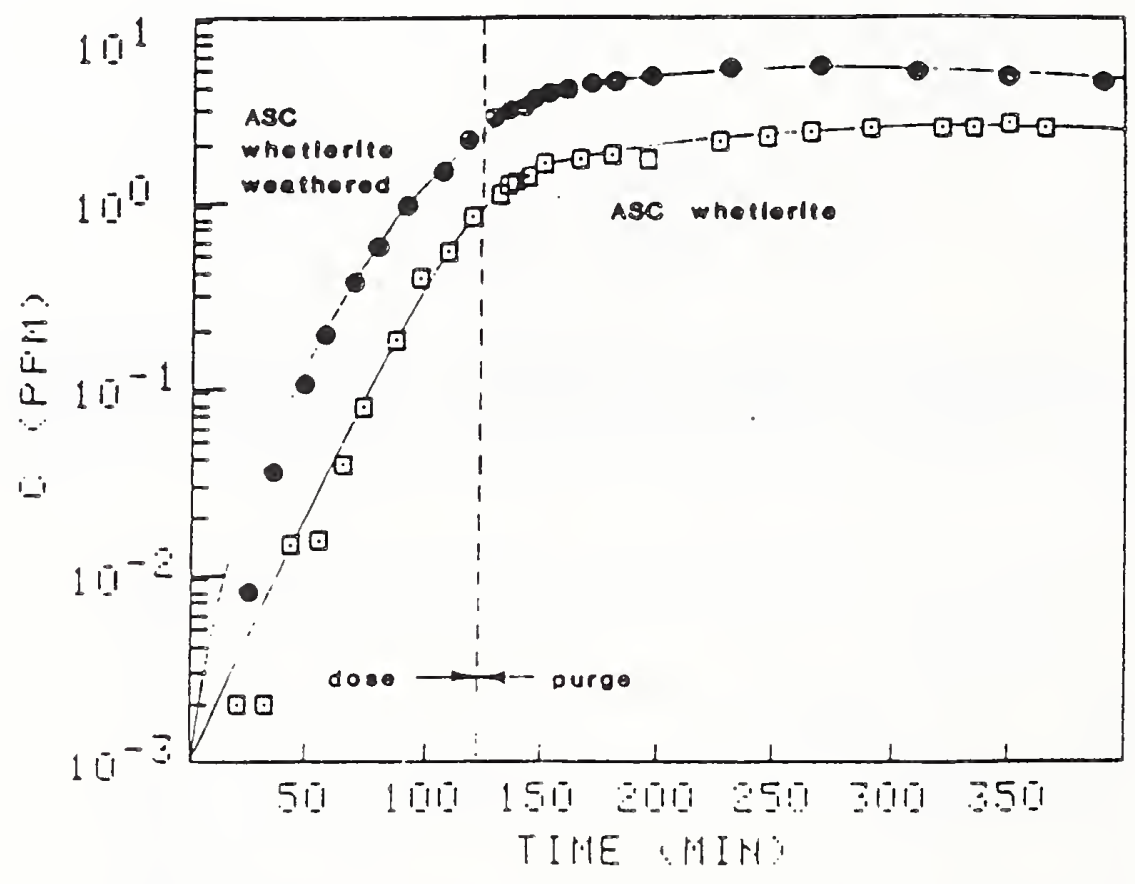

Figure 6: Penetration behavior of benzene-air flows through a new and a weathered ASC whetlerite

Another example of the above desorption behavior is the adsorption of methyl iodide on a nuclear grade carbon as shown in Figure 7. The dose into the air flow of $30 \% \mathrm{RH}$ lasted 120 minutes, but the peak concentration was observed at 1800 minutes and the effluent methyl iodide concentration $\left(0.001 \mathrm{mg} / \mathrm{M}^{3}\right)$ was detected after 5 days.

\section{Removal via Chemisorption}

Many toxic gases combine chemically with activated carbon and with impregnated carbons. For example, ozone and hydrazine vapors react directly with the carbon; hydrogen cyanide, however, is held by a cooperchromium impregnation on a coal-base activated carbon. When the products of a chemisorption process are retained by the carbon bed, a slow 


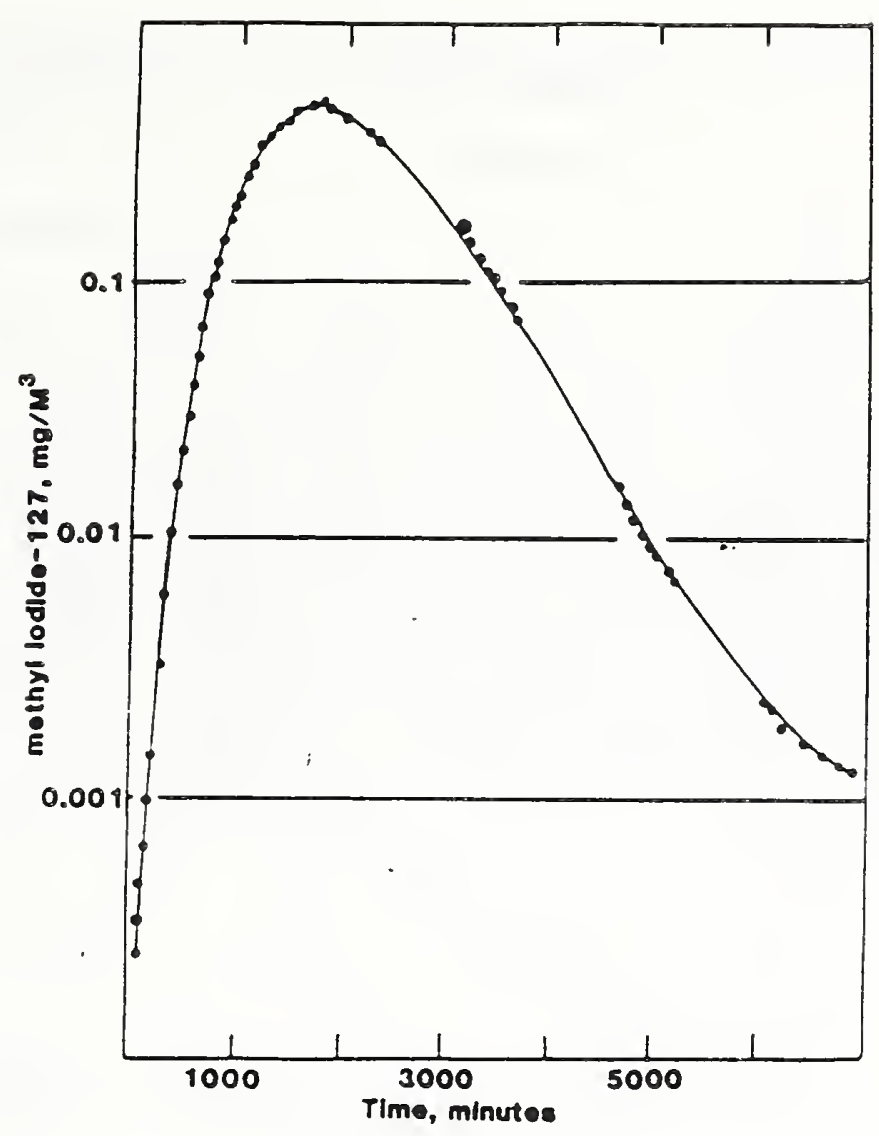

Figure 7: $127 \mathrm{ICH}_{3}$ in Effluent (mg/M $)$ for the prolonged ( 5 days) purge at $30 \%$ RH with NRL 5144 .

degradation of the removal efficiency takes place and the carbon bed must be replaced. Frequently, the chemisorption process may lead to a volatile product often toxic in itself. An example is the chemisorption of HCN by whet lerite charcoal (Figure 8) in which cyanogen ( $C N)_{2}$ is formed and is present in the effluent air flow. Protection against HCN was realized for more than 80 minutes, but the cyanogen broke through in one-tenth of the time. This example stresses the importance of the test gas that is selected to evaluate the efficiency of the ventilating system.

\section{Depth Profile within a Charcoal Bed}

A test procedure for activated carbons in service (used carbons) should reveal the residual life as well as the observed penetration of the test gas. Test procedures for new adsorbents are of value for procurement decisions, but the procedure must be modified for used materials.

The depth profile has been determined for a number of test beds containing nuc lear-grade activated carbons. The count profile in the penetration of radioactive methyl iodide-13l at equal increments of depth was determined and was found to be exponential along the line of flow. A large numerical magnitude of the slope (Figure 9) is characteristic of new and good carbons; a low value signifies poor retention by the test column.

As the time in service increases, the sequential count from inlet to out let sections at the sample may not decrease exponentially. In fact, fewer counts have sometimes been observed in the inlet sections than in the remaining sections. The presence of adsorbed contaminants is held 


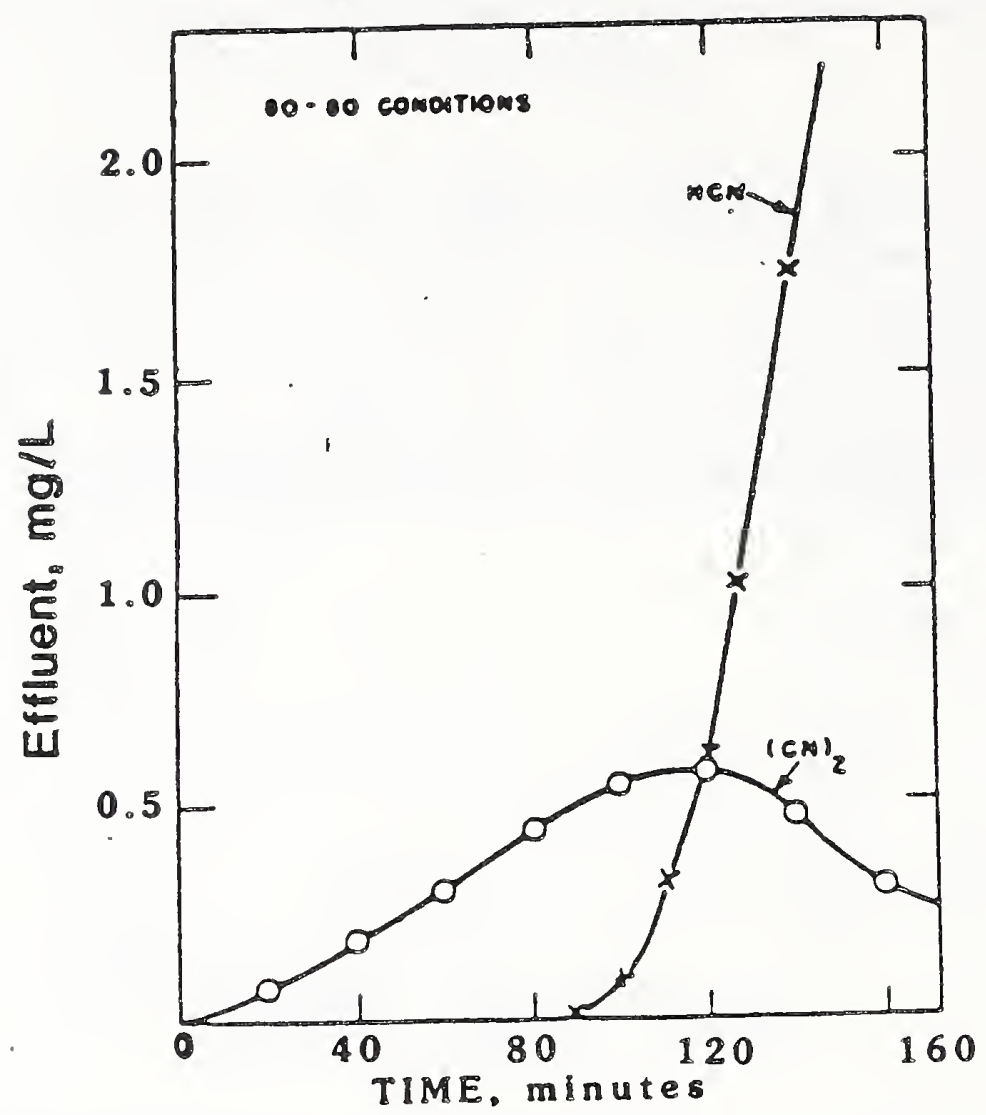

Figure 8: Effluent concentrations for HCN on Type A.

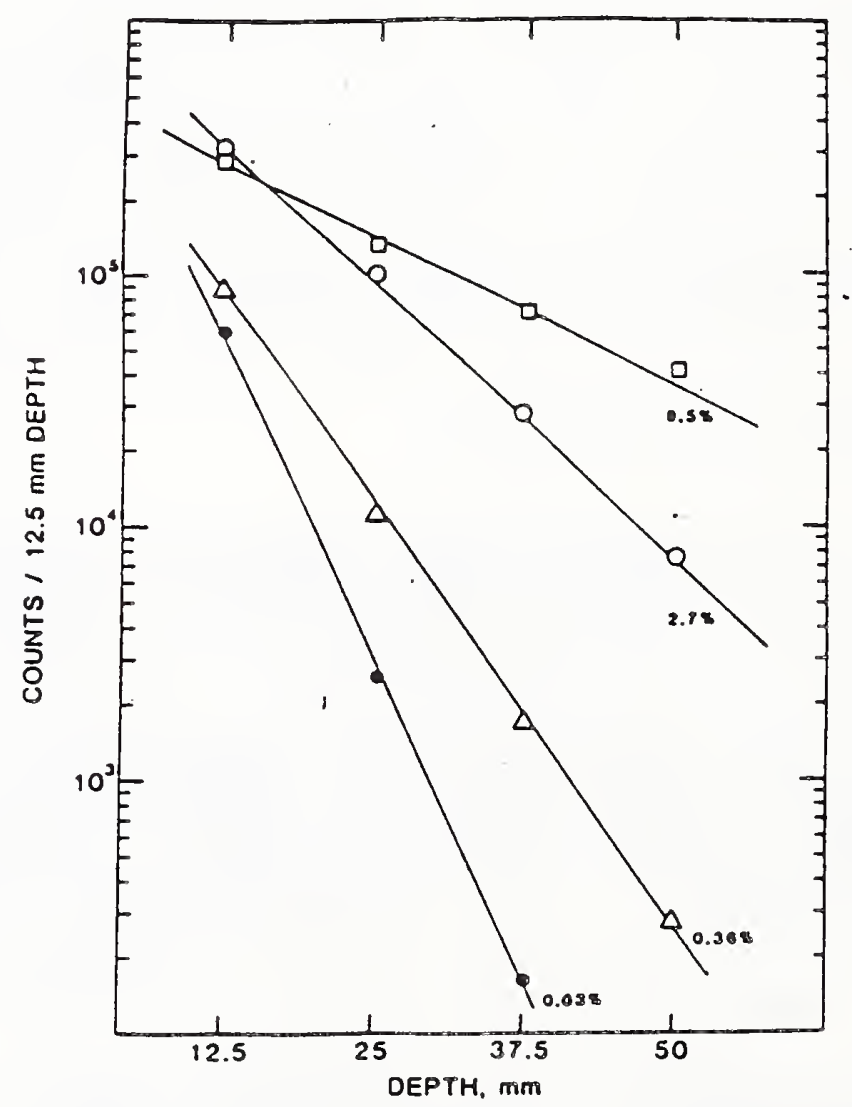

Figure 9: Progressive decrease of the count profile as the penetration of radioactive methyl iodide-131 increases. 
responsible for the migration of the radioactive methyl iodide-131 and such behavior can occur in other applications where charcoal is used in ventilating systems.

\section{Concluding Remarks}

It is evident that the application of an activated carbon adsorber for gaseous and vapor removal in ventilating systems can involve many chemical problems. The moisture content of the air and the atmospheric contaminants are bacikground considerations. The analysis of the building air can reveal the presence of those constituents easily removed and those removed with more difficulty. The design of the carbon bed depends on the desired level of removal. More than one type of test gas should be used to evaluate the carbon efficiency and its possible regeneration capacity. A carbon bed may be considered as a holding unit which only gradually released the undesirable gases and vapors at a lower concentration below an undesirable leve 1. There are, as usua 1, many compromises to be made in the ultimate design of the system. 


\author{
Amos Turk \\ Department of Chemistry \\ The City College of the City University of New York \\ New York, NY 10031
}

Evaluation of activated carbons or other granular media used for air cleaning requires testing both for efficiency and capacity.

Efficiency - Practically all granular media utilize one or a combination of three processes: (a) physical adsorption; (b) acid-base neutralization; (c) oxidation. The potential efficiencies of these actions in granular beds depend on their rates as well as on the bed characteristics. Physical adsorption requires contact between the molecules of gas or vapor and the adsorbent surface. The adsorbents commonly used for indoor air cleaning are granules of mixed sizes ranging in diameter from about 1 to $5 \mathrm{~mm}$, packed into beds of uniform thickness. Under such conditions, the halflife of a molecule in the gas phase before it reaches the surface of a granule is about $0.01 \mathrm{~s}$ [1]. Then, if the air stream containing contaminant molecules enters such a bed, the contact efficiency, E, the fraction of molecules that have contacted a solid surface after $n$ seconds is:

$$
E=1-2^{-100 n}
$$

From this equation, typical contact efficiencies are $75 \%$ for a residence time of $0.02 \mathrm{~s}, 93.75 \%$ for $0.04 \mathrm{~s}$, and $98.44 \%$ for $0.06 \mathrm{~s}$. However, such efficiencies are realized only with a fresh bed. As soon as some adsorption occurs, the mass transfer zone begins to move downstream.

Acid-base neutralization, which involves the transfer of a proton, is practically an instantaneous reaction, so it is not rate-limiting in granule beds. Furthermore, the neutralization of a base by a strong acid, or of an acid by a strong base, goes practically to completion. Thus, activated carbon impregnated with $\mathrm{NaOH}$ or $\mathrm{ROH}$ can remove weakly acidic gases such as $\mathrm{SO}_{2}$ or $\mathrm{H}_{2} \mathrm{~S}(2)$, and carbon impregnated with phosphoric acid can remove ammonia or amines.

Oxidation reactions, on the other hand, vary widely in rates, are generally slower than the actions afforded by physical adsorption or by acid-base neutralization, and are therefore usually rate controlling when they are used for air cleaning in granular beds.

Capacity of an air cleaning device using granular media can be determined by monitoring the medium for exhaustion or the effluent for breakthrough, or by using some other analytical system to predict breakthrough and thus estimate probable capacity. Monitoring could conceivably be carried out by observing color change, recording the change of mass of the medium with time, analyzing the effluent, removing test samples of the medium for analysis, or extracting parts of the air stream from within the bed for analysis. Carbon does not change color. Potassium permanganate (purple) is reduced to manganese dioxide (brown), but the surface color obscures that of the interior. Changes in mass due to saturation with organic vapors are obscured by gains and losses of moisture. Analysis of air 
streams after or within the bed, or testing of the medium itself, requires instrumentation that is usually impractical in domestic or commercial settings, but is appropriate in a testing facility.

It is sometimes helpful to carry out simultaneous competitive tests on the same air stream to evaluate different media, or to compare different impregnations for the same substrate. One suitable device [3] utilizes an air sampler comprising 16 respirator cartridges mounted on a manifold plate. The cartridges $c a n$ be loaded with different media and removed and tested from time to time for air cleaning efficiency or for degree of saturation. Alternatively, an air stream can be split between two larger units for direct one-on-one competitive testing. For devices that have low air cleaning efficiencies, either because of the inherent inefficiencies of their media or because of their bed characteristics, and which therefore are designed to hand le air in recirculation with multiple passes, tests with recirculation in closed systems are appropriate. The test protocol makes use of the first order decay relationship (4),

$$
C=C_{0} e^{-m E Q t / V}
$$

where $\quad C=$ vapor concentration at time $t$

$C_{0}=$ initial concentration

$Q=$ air flow rate

$m$ = mixing factor in the space

$E=$ efficiency of the unit

$V=$ volume of the space

To evaluate E, a combination of air cleaner and test gas that is known to be $100 \%$ efficient, such as activated carbon and $\mathrm{CCl}_{4}$ vapor, is used in a space of known volume for a specified time and flow rate, and the initial and final concentrations are measured. Then $E=1$ and the mixing factor in the space, m, can be calculated. Now the air cleaner to be tested is substituted under the same conditions and, knowing $m$, the efficiency of the unit can be determined.

New approaches for specific gases and vapors - The capacity of activated carbon for adsorption of vapors depends in part on their boiling points. In general, the lower the boiling point of a liquid, the lower is the capacity of activated carbon to retain its vapor by physical adsorption. When the boiling point is below about $50^{\circ}$ or $60^{\circ} \mathrm{C}$, activated carbon is usually impractical as a physical adsorbent. The common alternative is the use of a chemically reactive impregnant on activated carbon or other substrate. Examples are the use of permanganated-impregnated alumina for removal of formaldehyde by oxidation, or cautic-impregnated carbon for removal of hydrogen sulfide by neutralization. Such impregnations, however, occupy pore volume and surface area and thus limit the capacity of the substrate for physical adsorption of other vapors. One other possibility is the use of a gaseous reactant that is innocuous in itself and that does not remain on the adsorbent. A useful reactant for the removal of hydrogen sulfide and methyl mercaptan is ammonia gas (5), which acts as a catalyst for the oxidation of $\mathrm{H}_{2} \mathrm{~S}$ to sulfur by atmospheric oxygen. The ammonia can be used in concentrations below its odor threshold. A suggested mechanism for the reaction is:

$$
2 \mathrm{NH}_{3}+\mathrm{H}_{2} \mathrm{~S}=\left(\mathrm{NH}_{4}\right)_{2} \mathrm{~S}(1 \text {, fast equilibrium) }
$$




$$
\begin{aligned}
& \left(\mathrm{NH}_{4}\right)_{2} \mathrm{~S}+1.5 \mathrm{O}_{2} \rightarrow\left(\mathrm{NH}_{4}\right)_{2} \mathrm{SO}_{3}(2 \text {, rate controlling) } \\
& \left(\mathrm{NH}_{4}\right)_{2} \mathrm{SO}_{3}=2 \mathrm{NH}_{3}+\mathrm{SO}_{2}+\mathrm{H}_{2} \mathrm{O}(3 \text {, fast equilibrium) } \\
& 2\left(\mathrm{NH}_{4}\right)_{2} \mathrm{~S}+\mathrm{SO}_{2} \rightarrow 3 \mathrm{~S}+4 \mathrm{NH}_{3}+2 \mathrm{H}_{2} \mathrm{O} \text { (4, fast redox) } \\
& \text { Sum: } 3 \mathrm{H}_{2} \mathrm{~S}+1.5 \mathrm{O}_{2} \rightarrow 3 \mathrm{~S}+3 \mathrm{H}_{2} \mathrm{O}\left(\mathrm{NH}_{3}\right. \text { catalyst) }
\end{aligned}
$$

\section{References}

(1) A. Turk, H. Mark, and S. Mehlman, "Tracer gas nondestructive testing of activated carbon cells," Materials Res. \& Stds. 2, No. 11, 24 (1969).

(2) R. K. Sinha, M. J. Wagner, and R. J. Joyce, "Impregnated activated carbon for improved removal of malodorous compounds." U.S. Patent $4,072,479$ (1978).

(3) A. Turk, L. K. Oti, A. E. Steinberg, and T. E. Wolf, "Assessing the performance of activated carbon in the indoor environment," Am. Ind. Hyg. Assoc. J. 45, 714 (1984).

(4) A. Turk, "Measurements of odorous vapors in test chambers: theoretica1," ASHRAE J. 5, 10 (Oct. 1953).

(5) A. Turk, E. Sakalis, and J. Lessuck, "The use of ammonia and activated carbon for removing sulfur gases from air," Proc. Third Int 1. Conf. of Israel Soc. for Env. Quality, Jerusalem, June 11, 1986. 


\author{
Allan H. Frey, Ph.D. \\ Rand omline, Inc. \\ County Line and Mann Roads \\ Huntingdon Valley, PA 19006
}

\title{
ABSTRACT
}

The complex electrical fields that exist in al1 spaces interact with airborne charges, particulates, water droplets, and adsorbed gases. These interactions in large part determine the deposition of contaminants in and on people, objects, and walls in a room, as I described in a recent paper (Frey 1986a). Thus, such fields have substantial implications for the development of test procedures for the measurement of gaseous contaminants.

Recently published research has shown that in-duct electrical fields can be created which reduce the concentration of gaseous and particulate contaminants in a room. This also bears on the development of methodology for the valid measurement of gaseous contaminants. Reported here are data on the effect of in-duct electrical fields.

The objective of the first experiment reported here was to determine the mechanism by which in-duct electrical fields reduce the concentration of particulates in a room. The statistical analyses of the data indicate that the operation of these specific electrical fields in the duct significantly reduced the particle count in the room and suggest a mechanism for the effect. There was a shift in particle size spectrum that can be interpreted as the in-duct electrical field accelerating the normal coagulation of sma 11 particles into larger particles which are more readily trapped in the filter.

Since gaseous contaminants to a large extent adsorb on particulates, the second experiment was undertaken to gather information on the influence of these in-duct complex electrical fields on gaseous contaminants. The results with gases are consistent with the mechanism suggested by the particulate data. The concentrations of formaldehyde, ammonia, sulfur dioxide, and carbon dioxide were substantially decreased and the decrements were statistically significant. Thus, it appears that some gaseous contaminants can be controlled in the process of controlling particulates with this technique.

The third experiment was a practical application of the information gathered. We tested for and found that animal room odor concentration was decreased by passing the room air supply through in-duct complex electrical fields. The odor panel reported a substantial decrease that was statistically significant. Taken together, the results of these three experiments provide information which bear on methods of measurement of gaseous contaminants.

\section{INTRODUCTION}

The complex electrical fields that exist in all spaces interact with airborne charges, particulates, water droplets, and adsorbed gases. These 
interactions, in large part, determine the deposition of contaminants in and on people, objects, and walls in a room, as I described in detail in a recent paper (Frey 1986a). Thus, such fields have substantial implications for the development of test procedures for the measurement of gaseous contaminants. Complex electrical fields can be created in ducts, and they can modify the concentration of both gaseous and particulate contaminants in a room, as has been shown in recently published research (Frey 1983 , $1984,1985,1986 \mathrm{~b})$. It was estab Iished that such effects were not due to ionization or ozone. This also bears on the development of methodology for the valid measurement of gaseous contamiants. The three experiments reported here were directed toward obtaining data to begin to define the nature of the effect of in-duct complex electrical fields on gaseous and particulate contaminants and to test a practical application.

\section{EXPERIMENT I}

The objective of this experiment was to determine the mechanism by which in-duct electrical fields reduce the concentration of particulates in a room.

\section{Method}

The testing was carried out in a room $4 \times 11 \times 2.5$ meters high $(13 \times 36 \times 8$ fto). The room had its own closed circuit air handing system. Air entered the room through a pair of slot supply diffusers near the ceiling centerline, passed down through the room, and exited into a duct through a pair of slot return grilles at the bottom of the walls that were parallel to the diffuser slots. In the duct, the air passed sequentially through a smoking device, a 55 percent filter, a blower, three electrical field screens, and then re-entered the room through the supply diffusers. The room air change rate was 10 per hour with fifty percent recirculation. The air supply was filtered through activated alumina to remove moisture. The temperature was about $70^{\circ} \mathrm{F}$ with a relative humidity less than $3 \%$. Tests have also been run with a humidity of $50 \%$ and $85 \%$ with similar results. The air in the room was purged to the outside between test runs and said runs were separated by at least two days.

The electrical field screens installed in the duct were $60 \times 60 \mathrm{~cm}(2 \times 2$ ft.), except the center one which was $5 \mathrm{~cm}$ (2 in.) 1 arger. They were spaced $7.6 \mathrm{~cm}$ ( 3 in.) apart and were perpendicular to the air flow. The screens were made of braided wire configured as shown in Figure 1. Electric field generators supplied a $700 \mathrm{~V}$ peak to peak $177 \mathrm{kHz}$ signal that was applied to the center screen and also a $25 \mathrm{kV}$ DC signal that was applied to the other two screens. The current was less than $3 \mathrm{~mA}$. The smoke was generated by burning cigarettes in a smoking device in the duct. For a 75-minute period in each run a mean rate of $1044 \mathrm{mg} / \mathrm{min}$ of airborne burned cigarette products was produced.

Two particle measuring instruments were used concurrenty in the experiment. One was a Royco, Incos Model 218 Particle Monitor, which measured particle concentration and size by light scattering. The other instrument used was a California Measurements, Inc., Model PC-2 Aeroso 1 Particle Analyzer, which measures particle concentration and size by mass. It is a 10-stage cascade impactor with quartz crystal microbalance mass monitors in each state. This instrument provided a printout of the mass 


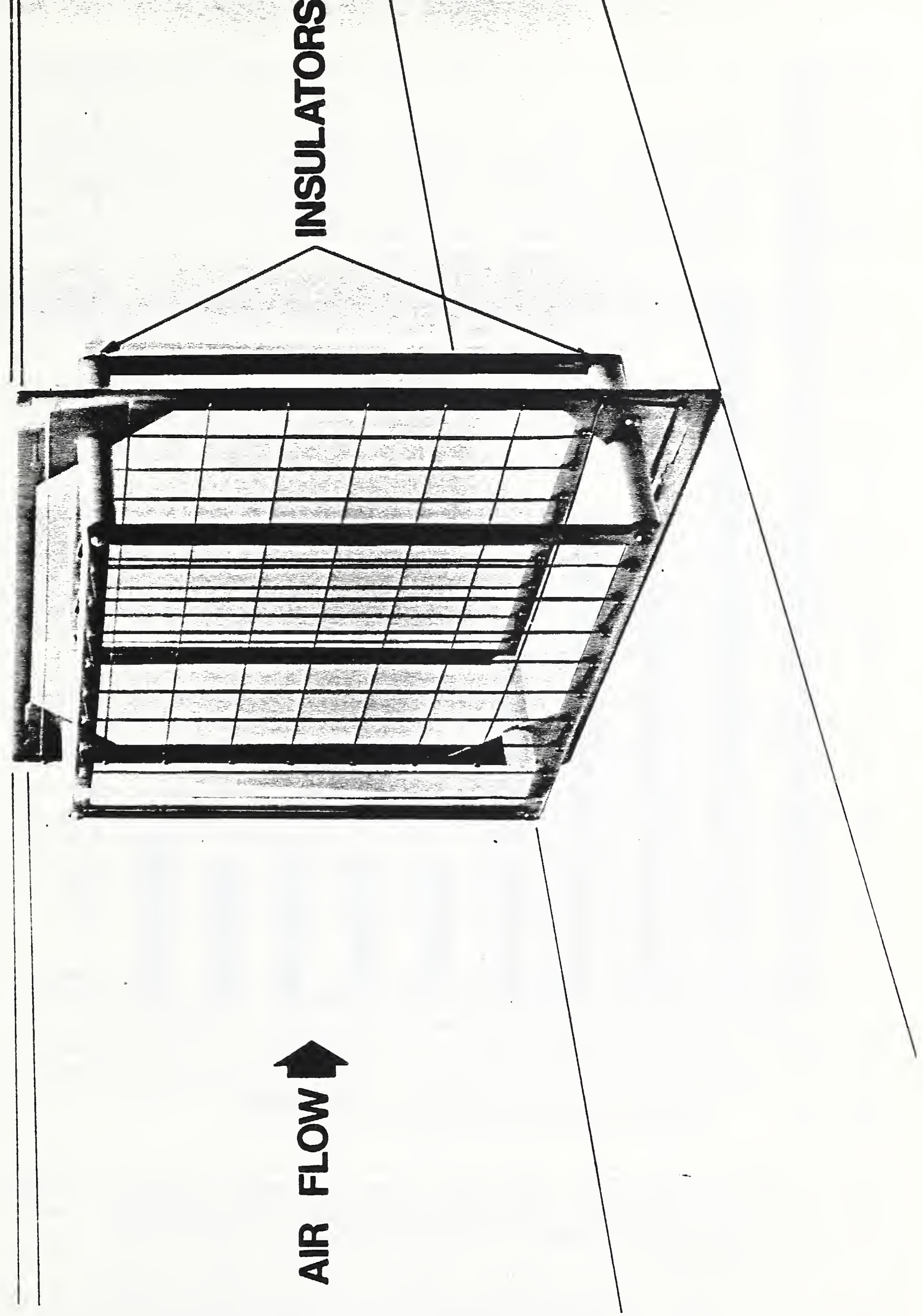

Figure 1. Electrical field screens 
for each stage at the end of each 180 second sampling period throughout each test run.

During each 90-minute test run, there was no smoke introduced into the air for the first 15 minutes. During the next 20 minutes, the smoking of the cigarettes began and the smoke distribution in the room was allowed to stabilize. During the remaining 55 minutes, the smoking was held at an essentially constant rate. Thus, the primary data for the analyses were the last 55 minutes of each experimental run.

A counterbalanced experimental design was used with the electrical fields off for days 1 and 4 and on for days 2 and 3 .

\section{$\underline{\text { Results }}$}

In accordance with the experimental design, the days 1 and 4 data (field off) gathered after smoke levels stabilized were combined and the days 2 and 3 data (field on) were combined.

The first objective in analyzing data was to see if the results from the two instruments were consistent. Since one instrument operates by 1 ight scattering and the other operates by mass measurement, one would expect substantial, though not complete, correlation in the results. The statistical analyses showed that both instruments indicated the electrical field in the duct was decreasing the particulate concentration in the room compared to the control situation.

A statistical analysis using the data from all ten stages of the impactor was carried out. The results showed there was a difference between the field on and off conditions, and it was significant $(t=5.02, p<0.005)$.

Statistical analyses were also done stage by stage. These showed that the operation of the in-duct electrical field substantially reduced the mass of small particulates in the air and slightly increased the mass of large particulates. This relationship is shown in Figure 2 as percent decrease or increase in mass from the mass determined in the electrical field off condition. The difference between field on vs. off data on each stage was statistically significant except for the 1.6 micron stage data.

Since the 1.6 micron size seemed to be the transition point, it was used as a break point in further analyses. The mean mass for the small particles ( 0.05 to 0.8 micron) was calculated. For the electrical field on condition, it was $0.232 \mathrm{mg} / \mathrm{m}^{3}$; and for off condition, it was $0.378 \mathrm{mg} / \mathrm{m}^{3}$. Thus, the operation of the electrical field reduced the mass of small particles in the room air to 61 percent of what it was in the field off condition.

The mean mass for the large particles $(3.2$ to 25.0 microns) was calculated. For the electrical field on condition, it was $0.033 \mathrm{mg} / \mathrm{m}^{3}$; and for the off condition it was $0.009 \mathrm{mg} / \mathrm{m}^{3}$. Thus, the operation of the electrical field increased the mass of the large particles in the air to $367 \%$ if what it was in the off condition.

Note that the loss of small particle mass is not balanced by the gain in large particle mass. The gain of $367 \%$ in large particle mass in the field 


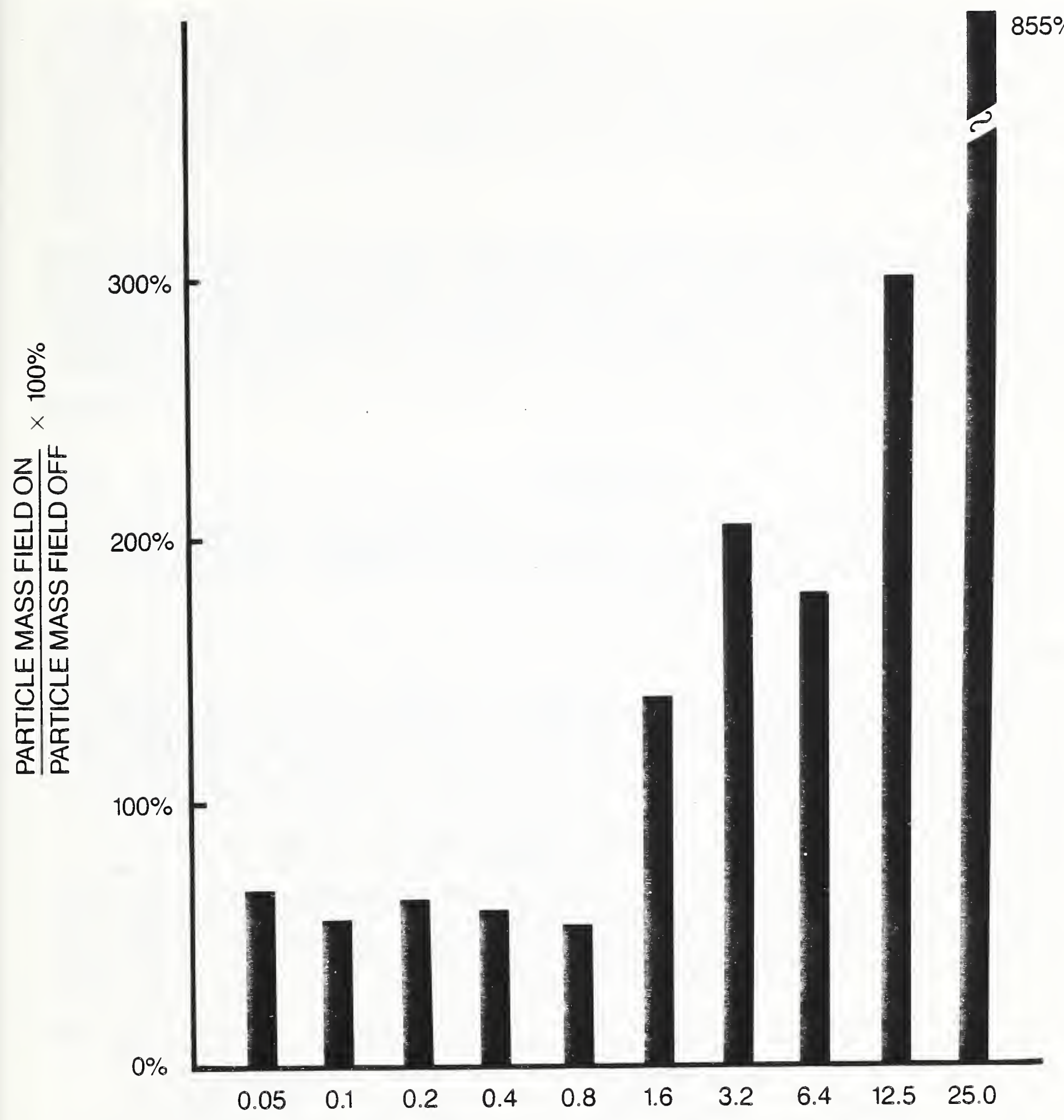

PARTICLE SIZE IN MICRONS

Figure 2. Percent decrement or increment of particle mass with the electrical field on when compared against the electrical field off data as the baseline. The field off data would al 1 be $100 \%$. The difference in mass between on and off conditions at each particle size are statistically significant, except at the 1.6 micron size. 
on condition accounts for on 1 y $6 \%$ of the mass 1 ost in sma 11 particles. The other $94 \%$ of the decrement in smal1 particle mass is going elsewhere. In view of the fact that there is a significant increase in large particles, these data could be interpreted as indicating that the $94 \%$ of small particle mass that is unaccounted for is being deposited in the filter.

\section{Discussion}

The statistical analysis of the Royco data indicated that the operation of these specific electrical fields in the duct significantly reduced the particle count in the room. The statistical analyses of the cascade impactor data support that conclusion and add further information that suggests a mechanism for the effect. The shift in particle size can be interpreted as the operation of the electrical field to accelerate the normal coagulation of small particles into larger particles which are more readily trapped in the filter.

\section{EXPERIMENT 2}

In view of the findings on particulates, a complementary set of experiments were undertaken to gather information on the influence of these in-duct complex electrical fields on gaseous contaminants. Gaseous contaminants to a large extent adsorb on particulates.

\section{Method}

The gases used were formaldehyde, ammonia, sulfur dioxide, and carbon dioxide. The testing was carried out in a room $2.74 \times 5.79 \times 2.44$ meters ( $9 \times 19 \times 8 \mathrm{ft}$. high) with a floor of vinyl tile and walls and ceiling paneled. The paneling was coated with polyurethane varnish and the joints were sealed with duct tape. The room had its own closed circuit air handling system. Air entered the room through supply diffusers at one side passed across the room, and exited into a duct through return grills at an air change rate of 21 per hour. In the duct, the air passed sequentially through a $55 \%$ filter, three electrical field screens, the blower, and then re-entered the room through the supply diffusers. The temperature was about $70^{\circ} \mathrm{F}$ with a relative humidity bout $65 \%$.

There were two series of experiments using these gases. In the first series, the initial gas concentrations were set at levels at which the effects on people are just noticeable. In the second series, the initial gas concentrations were set at approximately hazardous levels for short exposure. A Gastec gas detector tube system was used as the measuring instrument. In the first series, two evaluators independently read each tube; one evaluator had no knowledge of the test conditions, so the experiment was double-blind for him. Because of the reliability of the evaluators, as noted in the results, there was only one in the second series.

The procedure was that the air handing system was turned on and the air in the test room purged to the outside for 30 minutes. This reduced the concentration of the gas of interest to normal ambient as verified by a Gastec tube measurement at the end of each purge. Agas, such as sulfur dioxide, was then injected into the test room. Sufficient gas was released to bring the concentration up to approximately the predetermined standard 
concentration used in the test. At this point, the gas was turned off and a 60-minute run was started. The gas concentration was measured with the Gastec tubes five minutes after injection stopped, 30 minutes into the run, and at the end of the 60 minute run. At the end of each run, the room was purged to baseline concentration and the next test in the series was then begun. There were twelve runs in each series of experiments for each gas, six with the fields on and six with them off. The runs were done in an ordered, counterbalanced sequence.

The sulfur dioxide and carbon dioxide were injected into the center of the room via a hose connected to a cylinder of gas located outside the room. The ammonia and formaldehyde were injected into the room with a Pasche model H airbrush spraying a 10 percent formaldehyde solution or an ammonium hydroxide solution.

\section{Results}

The first question addressed was the reliability of the readings of the Gastec tubes. Pearson product-moment correlations were computed between the data provided by evaluators 1 and 2. There was near perfect correlation in each set of their readings $(r>0.95)$. This indicates that they were reliably reading the Gastec tubes and were doing so without bias.

There was natural decay in gas concentrations over time without the electrical field on. Thus, for clarity of presentation, the data are presented as the percent reduction in the field-on condition compared to the control (field-off) condition. For testing the significance of the differences between conditions, an analysis of variance was done on the data. The results of the statistical analyses for each of the gases are shown in Tables 1 and 2 .

\section{TABLE 1}

Reduction of gas concentration by use of in-duct complex electric fields when compared to controls. The initial concentrations were set at levels that would produce the first noticeable effects in people. The significance levels were determined by analysis of variance.

Gas

End of 30 Minutes

End of 60 Minutes

Mean Percent Reduction

$\mathrm{CH}_{2} \mathrm{O}$ 26

0

0
Signif.

0.001

0.01

ns

ns
Mean Percent Reduction Signif. 39 .01 .001 .05

11 .01

The mean concentration at the 5,30 , and 60 minute points were formaldehyde $\left(\mathrm{CH}_{2} \mathrm{O}\right): 2.8,2.5,1.5 \mathrm{ppm}$; ammonia $\left(\mathrm{NH}_{3}\right): 24.7,14.4,7.5 \mathrm{ppm}$; sulphur dioxide $\left(\mathrm{SO}_{2}\right): 25.3,18.7,14.3 \mathrm{pm}$; carbon dioxide $\left(\mathrm{CO}_{2}\right): 2.4,1.9,1.5 \%$. 
Reduction of gas concentration by use of in-duct complex electrical fields when compared to controls. The initial concentrations were set at levels that would be hazardous to people when exposed for a short period. The significance levels were determined with use of the analysis of variance.

Gas

End of 30 Minutes

End of 60 Minutes

Mean Percent

Reduction

Mean Percent

Reduction

Signif.

$\mathrm{CH}_{2} \mathrm{O}$

39

.001

49

.01

$\mathrm{NH}_{3}$

0

ns

25

.001

$\mathrm{SO}_{2}$

13

.01

14

.001

$\mathrm{CO}_{2}$

10

.01

13

.001

The mean concentration at the 5,30 , and 60 minute points were formaldehyde $\left(\mathrm{CH}_{2} \mathrm{O}\right): 4.3,2.0,1.3 \mathrm{ppm}$; ammonia $\left(\mathrm{NH}_{3}\right): 39.6,24.6,14.1 \mathrm{ppm}$; sulphur dioxide $\left(\mathrm{SO}_{2}\right): 49.8,40.5,32.9 \mathrm{ppm;}$ carbou dioxide $\left(\mathrm{CO}_{2}\right): 4.8,4.0$, $3.4 \%$.

\section{Discussion}

The data on gases extend the finding that passing room air through in-duct complex electrical fields has a statistically significant effect on contaminants. The extent and rate of the effect on the concentration of gaseous contaminants varies as a function of which gas is used. The extent of the effect, with at least some gases, is so substantial that it should be of practical significance.

\section{EXPERIMENT 3}

The objective of this experiment was to determine if the perception of animal room odors, which have a substantial ammonia component, would be influenced by passing the room supply air through an in-duct complex electrical field.

\section{Methods}

The exposure room was approximately $7 \times 5.5 \times 3$ meters high $(23 \times 19 \mathrm{ft}$. by $10 \mathrm{fto})$. Within this room was a $3.5 \times 3.5$ meter $(12 \times 12 \mathrm{ft.})$ clean room enclosure made of vinyl nylon fabric. Approximately 48 rabbits and $\mathrm{s}$ ix cats lived within the clean room enclosure at the time of each subexperiment and provided the odor. The air within the enclosure was changed 170 times per hour. This was accomplished by blowing ambient room air through a prefilter, the electrical field screens, a HEPA filter, and then through a vinyl nylon fabric duct into the enclosure. The air blown into the enclosure was then exhausted into the exposure room through an opening between the enclosure skirt and floor. The exposure room had an air circulation system which normally involved 17 air changes per hour. Air from the common building ventilation system was blown into the room. This 
mixed with the air exhausted from the clean room enclosure and the mixture in part exhausted from the room and in part recirculated through the screens and into the clean room enclosure. During this experimentation, the room air change rate was reduced to seven changes per hour (according to the building engineer) by partially blocking the exhaust ducts in the room. This was done to increase the odorant level. Except for the last sub-experiment, the temperature in the exposure room was $70^{\circ} \mathrm{F}$ and the relative humidity was $50 \%$. At the last sub-experiment, the temperature was $75^{\circ} \mathrm{F}$ and the relative humidity was $52 \%$. The electric field generator supplied a $700 \mathrm{~V}$ peak-to-peak $0.177 \mathrm{MHz}$ signal that was applied to the center screen, and a $25 \mathrm{kV}$ DC signal applied to the two outside screens that were in the duct.

An odor pane 1 consisting primarily of male college students was formed. The panel was trained in odor intensity discrimination using several odorants, e.g., amyl acetate, butyl alcohol. They were trained to make odor intensity discriminations using as a scale a graduated series of odorant concentrations in a series of ten identical amber $250 \mathrm{cc}$ bottles. Each bottle held $10 \mathrm{cc}$ of liquid, consisting of the odorant and sufficient solvent to bring the volume up to standard. These ten bottles were held in a rack in order of concentration and thus odor intensity.

After initial intensity discrimination training which involved scaling bottles of unknown odor intensities, the subjects were moved to the second state of intensity discrimination training. In this stage, the unknown odorant intensity was presented as an airborne odorant in the exposure room and the scale bottles were located outside of the room. The subject now walked into the exposure room in order to smell the odorant that was in the room. He then walked out of the room and smelled the odor in any of the scale bottles he wished in order to select which was most similar in intensity to the intensity of the one in the room. The order of unknown intensities presented in the room was determined by using a table of random numbers. After this second stage was completed, the hypothesis under consideration was tested.

The trained subjects were their own controls within the experimental design, and they did not know the conditions of the experiment. The experimentation consisted of four sub-experiments. The 20 minute cycles called for in the design allowed the use of four subjects in each subexperiment. The electrical field alternated between off and on for a total of five periods, each consisting of two cycles.

Animal room odor is complex, so we prepared an aqueous extract of pan sweepings to make up our scale. A twenty-step scale was used. The odorant prepared consisted of distilled water extract of rabbit pan sweepings which included the wood chips used as bedding, urine, feces, and also some of the alfalfa food which was always available in the cages. Because of deterioration of the scale odorants after several days, a new odorant scale was prepared from extracts of fresh pan sweepings before each subexperiment.

The subjects were briefed prior to each experiment. It was made clear to the subjects that they were the measuring instruments in this situation. It was emphasized that on the basis of their judgments, important decisions would be made concerning the nature of odor perception. In this way, their 
responsibilities were emphasized and their interest and motivation were peaked. They were asked not to discuss the experiment with other subjects.

Each subject, individually with the experimenter, walked into a small room (bottle room) off the subject waiting room where he smelled from various bottles of the scale for reference. He then walked with the experimenter approximately 12 meters (40 ft.) and through two doors to the exposure room. The experimenter would open the door to the exposure room and the subject would step in five feet to a standard position two feet from the clean room enclosure. the experimenter closed the door, the subject smelled the air, and they then returaed to the bottle room. There the subject would sniff several scale bottles and pick one whose odor intensity matched that of the room. Then the experimenter and the subject would repeat the procedure. This was done with each subject in turn and a cycle with a 11 subjects was completed within 20 minutes. At the end of 20 minutes, a new cycle began.

It was explained to the subjects that we would not make any deliberate changes in exposure room odor level between each pair of judgments which were normally separated by a minute or so in each cycle. They were told that changes in odor intensity might be made, however, on a pre-determined schedule after certain of the cycles.

At the end of each day's experimental session, the subjects were separately debriefed by the experimenter to gather additional information.

\section{Results}

Using the combined data from pre-determined test points, i.e., cycles prior to period change, the independent sub-experiments $1 ., 2$, and 4 data were evaluated and the nul I hypothesis was rejected at the .001 significance level using the Wilcoxon matched-pairs signed ranks test. This indicated that the in-duct electrical field did have an effect on perceived odor intensity. The effect amounted to a reduction by half in perceived odor intensity.

Sub-experiment 3 data is not included in the analysis. Two-thirds of the way through the data collection in sub-experiment 3 , the experimenter noticed that the data indicated there was little odor in the room in the control as well as in the test condition. This suggested that the exhaust ducts in the room were not blocked as they should have been. It was found, on investigation, that the building engineer had not blocked the ducts due to a scheduling error. Thus, sub-experiment 3 was discontinued, and the data discarded as not being valid within the context of the experimental design.

Some rather useful incidental information, however, was obtained from subexperiment 3. This amounted to a double blind experiment and it showed that the experimenter was not inadvertently biasing the subjects. The fact that the fault in the air handling system could be identified from the data indicates that the subjects were fulfilling their role quite well.

\section{Discussion}

It is clear that the complex electrical field significantly reduced the 
perceived odor in the test room. The reduction in this particular set of circumstances is equivalent to reducing the perceived concentration of odorant by about 50\%. According to the subjects at the final debriefing, this reduction was enough to change an unpleasant room to an acceptable one.

It appears that it takes approximately 45 minutes of air recirculation through the field to make such a reduction in perceived odor. The effect continues for approximately 20 to 30 minutes after the electrical field is turned off. It should be kept in mind that these numbers are specific to this test situation.

\section{CONCLUSIONS}

In-duct complex electrical fields can significantly influence the concentration of contaminants in a room. Both particulates and gases are affected. The data from these experiments suggest that the fields accelerate the normal coagulation of small particles into large particles. This would result in the particulates and the adsorbed gases being able to escape the normal electrostatic forces that exist in all rooms. The air currents could then carry them to the filter to be trapped.

Disclaimer - NBS does not endorse commercial products. Responsibility for the information included here rests solely with the manufacturer. The information is included here because it is deemed useful to the objectives of the symposium.

\section{REFERENCES}

Frey, A. H. 1983. Modification of animal room odor by passing the room supply air through a complex electrical field. Bulletin of Environmental Contamination and Toxicology, 31(6), 699-704.

Frey, A. H. 1984. Change in room aerosol concentration by in-duct complex electrical fields. The Journal of Environmental Sciences, Jan./Feb. $34-36$.

Frey, A. H. 1985. Modification of aerosol size distribution by complex

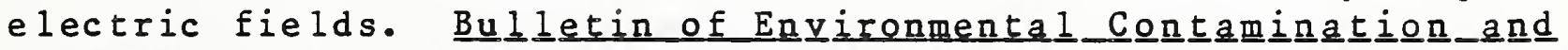
Toxicology, $34,850-857$.

Frey, A. H. 1986a. The influence of electrostatics on aerosol deposition. ASHRAE Transactions 1986, 92 (Part 1B), 55-64.

Frey, A. H. 1986b. Reduction of formaldehyde, ammonia, $\mathrm{SO}_{2}$ and $\mathrm{CO}_{2}$ concentrations in air. Journal of Environmental Sciences, July/Aug., $57-59$. 


\author{
Steve L. Zeis \\ Purafil, Inc. \\ Box 8043 \\ Atlanta, GA 30366
}

There are two principal types of gas-phase air purification media in general use for indoor air quality (IAQ) concrol today.

Activated carbons are high surface area (1000 to $1300 \mathrm{~m}^{2} / \mathrm{g}$ ) coconut shell or bituminous coal based adsorbents. These materials are generally not impregnated with chemical reagents and, within certain limits, are considered to be reversible adsorbents.

Impregnated aluminas are moderate surface area (250 to $350 \mathrm{~m}^{2} / \mathrm{g}$ ) alumina pellets, impregnated with one or more chemical reagents. The most commonly used impregnant is potassium permanganate $\left(\mathrm{KMnO}_{4}\right)$. These media are considered to be irreversible adsorbents, or chemisorbents.

To discuss these materials, three terms must be clarified:

Adsorption - the adherence of the atoms, molecules, or ions of a gas or liquid to the surface of (usually) a solid. Adsorption depends on the surface area of the adsorbent, temperature, pressure, the molecular weight and partial pressure of the adsorbate.

Absorption - the penetration of one substance (usually a gas or vapor) into the inner structure of another (usually a liquid) as $\mathrm{CO}_{2}$ is absorbed in water. . forming "Soda Water".

Desorption - the reverse of either adsorption or absorption, the process of removing an adsorbed material from the surface on which it is held, or an absorbed material from its absorbent. Desorption is accomplished by heating, reduction of pressure, by the presence of a more strongly adsorbed substance, or by a combination of these methods.

The reversible carbons operate purely by adsorption and, either factors being equal, the higher the internal or pore surface area, the higher the capacity. Reversible media tend to favor high molecular weight compounds (complex organics and heavy hydrocarbons), and have higher capacities at higher concentrations. Carbons are generally preferred for IAQ control when most contaminants are about 100 molecular weight, above about 5 ppm $v / v$, or both.

In irreversible media, given sufficient residence time, chemical contaminants that react with the impregnant will be modified and bound in the pellet, preventing desorption. For example, in $\mathbb{R} M_{n} 04$ impregnated a luminas, formaldehyde (HCHO) is first adsorbed onto the surface of the pores in the pellet, then adsorbed into solution with the $\mathbb{R}_{n} 04$ where a chemical reaction takes places, yielding water, carbon dioxide, potassium hydroxide and manganese dioxide. 


\section{TEST METHODS}

For reversible adsorbents, there is no chemical technique for determining when or whether a sample from an adsorbent device is saturated with one or more air contaminants under normally varying conditions of temperature, relative humidity, and contaminant character and concentration.

Research performed under ASHRAE sponsorship, on test method development, confirmed the variance of reversible media performance and capacity as these operating conditions change.

At the present time, to determine when to replace a reversible media bed that is being used to control one or more specific contaminants, the air in the building must be sampled and analyzed for those contaminants.

This can be done chemically, for a finite number of contaminants, but normally building operators wait until occupant complaints become numerous (or loud) enough before taking action.

Since irreversible media depend on chemical reagents to react with, and bind contaminants, sample or partially used media may be analyzed for the remaining reagent and the remaining life, predicted by comparing the amount remaining with the reagent present in a new sample.

Manufacturers publish curves developed empirically from a combination of $1 \mathrm{ab}$ and field experience to project a replacement date for impregnated aluminas.

Systems designers, specifiers and building operators need test methods and/or standards of performance that will allow them to know how a given system will perform and when the media must be replaced.

Presently, for reversible media systems, either air sampling and analysis must be performed periodically, or the building operator must depend on his nose, or on the noses of the building occupants.

Irreversible systems are a little more sophisticated, since a competent chemical laboratory can analyze a sample of media for the concentration of the active chemical reagent, but how does the building operator know if the system really removes the contaminants with which he is concerned?

How does the system designer or specifier know what to use and how much to use?

Standard test methods, comparable to the ASHRAE test method for particulate filters, would remove much doubt as to possible bias on the part of the manufacturer and would allow a designer or building owner to meet a "Standard" level (or one of several levels) of air quality, without resorting to complex, expensive air sampling and analysis.

Disclaimer - NBS does not endorse commercial products. Responsibility for the information included here rests solely with the manufacturer. The information is included here because it is deemed useful to the objectives of the symposium. 


\author{
Basil G. Louros \\ Sales Manager \\ Unisorb Corporation \\ South Houston, TX 77587
}

\title{
I. INTRODUCTION
}

Granular activiated carbon or alumina impregnated with potassium permanganate have been used to remove corrosive gases from commercial and industrial plant atmospheres. The corrosive gases interfere with the operation of sensitive computer and other instruments by corroding terminals resulting in costly downtime. These media are also used to remove noxious gases from work environments for personnel safety and protection.

\section{PROBLEM}

Once a corrosion or odor removing system has been installed the problem has been to change the media frequently enough so that the instruments will continue to be protected with time. What is needed is a quick and easy determination of the breakthrough front which will project media consumption and also determine any effects of any corrosive gas spilis in the plant environment. It is important that breakthrough of the media be avoided to protect equipment and personnel.

\section{Concept}

The mass transfer zone is defined as the depth of the media bed where the media goes from complete exhaustion to $100 \%$ activity. For most vapor phase applications we believe this mass transfer zone to be anywhere from 1 to 3 inches. The mass transfer zone (MTZ) is determined by activity or chemical remaining tests. These tests are currently done in the laboratory. and are time consuming and expensive. A quick reliable field test method is needed to assist the plant operator.

Samples are obtained from the last inch of a given packed bed in the direction of air flow since that is the only critical area in filtration. As long as the last inch has $100 \%$ activity remaining, this system is still efficient at $100 \%$. The Unisorb sampling technique is to use a sample thief to remove a representative sample and then analyze the sample depending on the given media. For activiated alumina impregnated with permanganate the sample was reacted with ferrous ammonium sulfate and back filtrated with potassium permanganate solution. The test is carried out with a Unisorb Test Rit and a colormetric method is used. The color intensity of the filtrate from a Mark 2 (permanganate impregnated alumina) sample is measured in a colorimeter, the reading is then compared to a known standard to determine the remaining chemical activity. Figure 1 shows the $\mathrm{R}-20$ meter reading as compared with the percent by weight on a dry basis of chemical activity remaining on the alumina.

This colormetric method offers fast, reproducible results in a portable equipment which is not affected by moisture in the sample and minimum error in the test procedure. Results from analytical and colormetric methods 
agree in the high range within experimental errors, that is, a range from $1 \%$ to $4 \%$. If the sample is less than $1 \% \mathrm{KMNO}_{4}$ analytical method will give a higher reading than the colormetric method. We believe this discrepancy in the lower range is probably due to interference between test solutions of the analytical method and chemicals adsorbed onto the sample surface. Therefore, we believe the colormetric method to be more indicative of the remaining activity.

\section{TEST METHODS FOR ACTIVATED CARBON}

For activated carbon the standard for activity has been carbon tetrachloride adsorption as measured in a complicated laboratory procedure. The colormetric test developed by Unisorb is again carried with the Unisorb Test Rit and involves treating a ground sample of activated carbon with a fixed amount of Methylene Blue Solution. The color intensity of the filtrate is then measured in a colorimeter and co-related with an equivalent carbon tetrachloride number for interpretation of the remaining capacity. Figure 2 shows a correlation curve between Methylene Blue number and carbon tetrachloride activity.

In comparison the colormetric method offers a quick convenient field testing method and is repeatable and accurate within a 10 to $15 \%$ range. No elaborate setup is required and all interferences by humidity have been taken into account in the correlation curve.

\section{CONCLUSION}

Both colorimetric methods offer quick reproducible results in the field for accurate determination and prediction of media bed breakthrough. For more information on the Unisorb model $\mathrm{R}-20$ Field Test Rit, please contact Unisorb Corporation, 1310 Genoa, South Houston. 


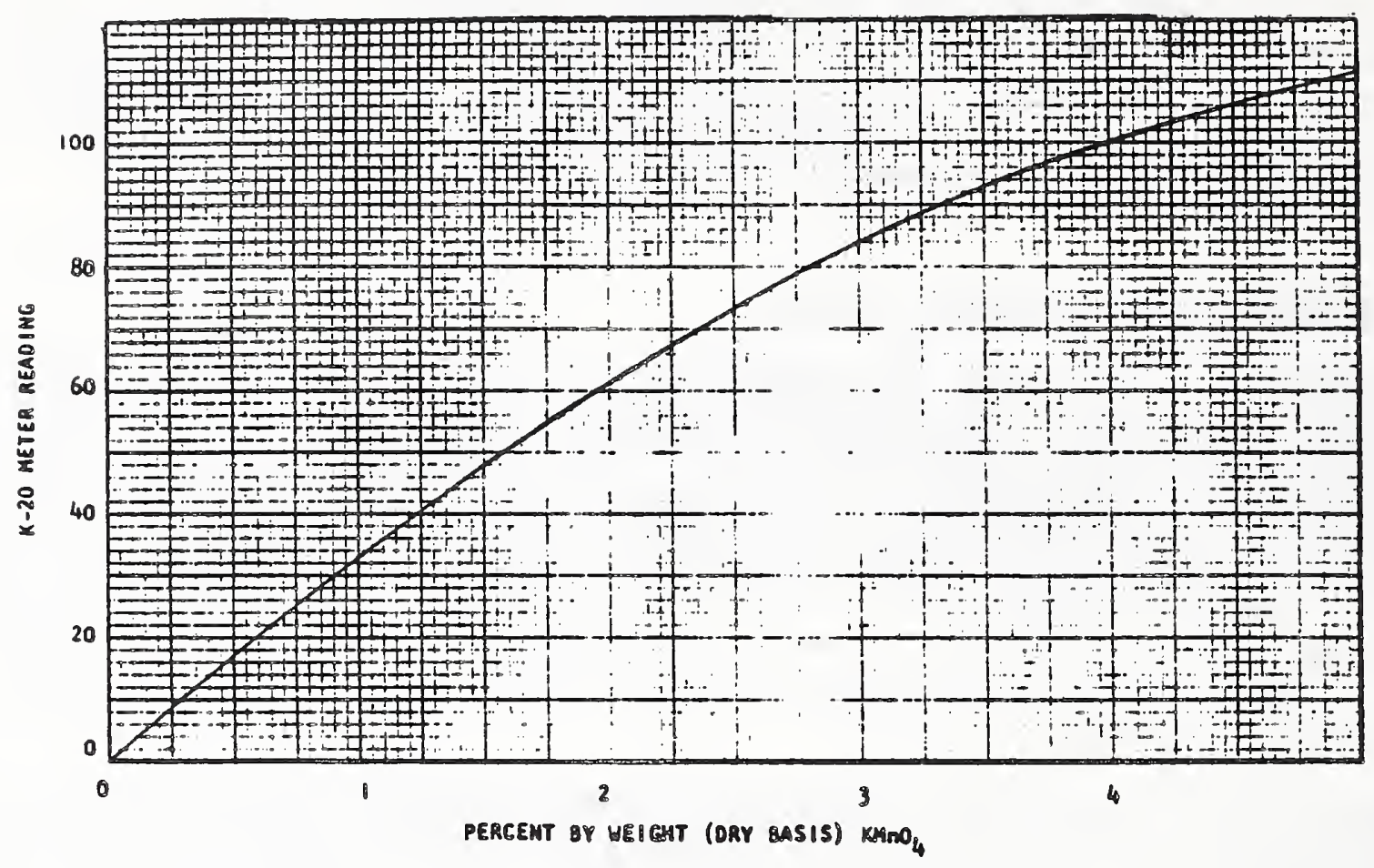

Figure 1

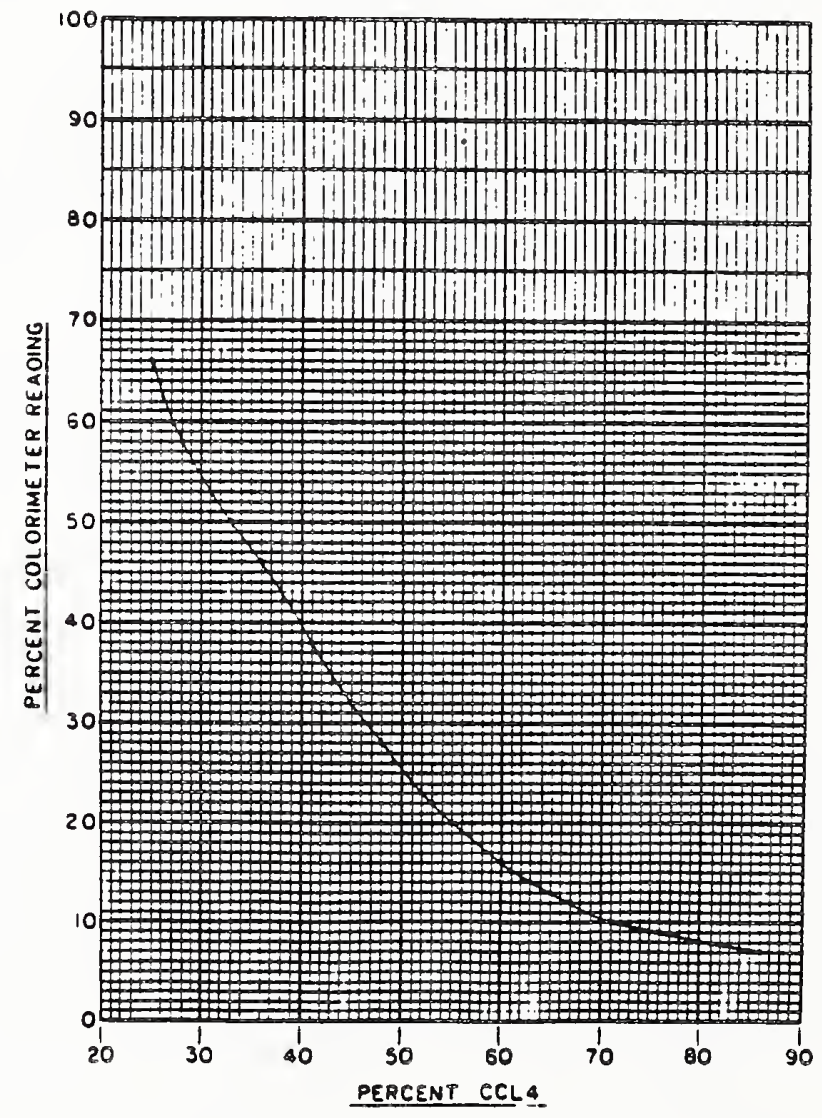

Figure 2

Disclaimer - NBS does not endorse commercial products. Responsibility for information is included here rests solely with the manufacturer. The of the symposium. 
CHARACTERIZATION OF THE LTC CATALYST:

Performance Against Common Air Pollutants

Marcia F. Collins

Research \& Development, Teledyne Water Pik

1730 East Prospect St., Fort Collins, Colorado

80525

\section{INTRODUCTION}

The Environmental Protection Agency has been conducting various studies of indoor air contaminants and their exposure $[1,2,3]$. These results and others indicate that personal exposure to specific pollutants is often to significantly greater concentrations than outdoor exposure limits. Tightening up of residences for reasons of energy conservation, greater use of synthetic materials in building and furnishing interiors, tobacco smoking, and increased use of solvents all are major contributors to the deterioration of indoor air quality.

Most individuals spend up to 90 percent of their time indoors; about 70 percent is in residential and office environments. The various types of pollutants -- as particulate, or harmful gases -- have been linked to ill health effects. Currently, the most controversial issue is the effect of sidestream tobacco smoke on non-smokers in the same environment.

As reported in an earlier paper [4], Teledyne Water Pik has developed a novel room temperature catalyst (ITC) for removal of various gaseous contaminants. In particular, this catalyst oxidizes carbon monoxide to carbon dioxide at very efficient rates. It also has shown good rates of removal for ozone, nitrogen and sulfur oxides, hydrogen sulfide, ammonia, and certain toxic components of cigarette smoke.

\section{LTC CATALYST MATERIAL}

In general, the LTC catalyst consists of a solution of copper and palladium salts deposited on a porous alumina substrate. It appears that this substrate material provides a certain enhancement of catalytic activity due to its specific surface chemistry, as well as large surface area for dispersion of the LTC composition. Also a minimum of post-deposition water content, tightly held by the alumina, is necessary to achieve reasonable rates of catalytic efficiency.

\section{Reaction Mechanism}

The reaction of LTC with carbon monoxide is analogous to the Wacker commercial process for producing acetaldehyde [5]. The oxidation-reduction cycle requires exchange of electrons between the copper and palladium metals of the complex; water is both consumed and returned to the catalytic cycle.

A simplified diagram of this reaction is shown in Figure 1. The depositions of metal salts contain activated complexes which create sites for selective chemisorption of oxygen and carbon monoxide. Present theory holds that "islands" of atomic oxygen are adsorbed, surrounded by carbon monoxide molecules and that the oxidation reaction occurs at their 
iface [6]. The rate of reaction is determined by the catalyst's ciency.

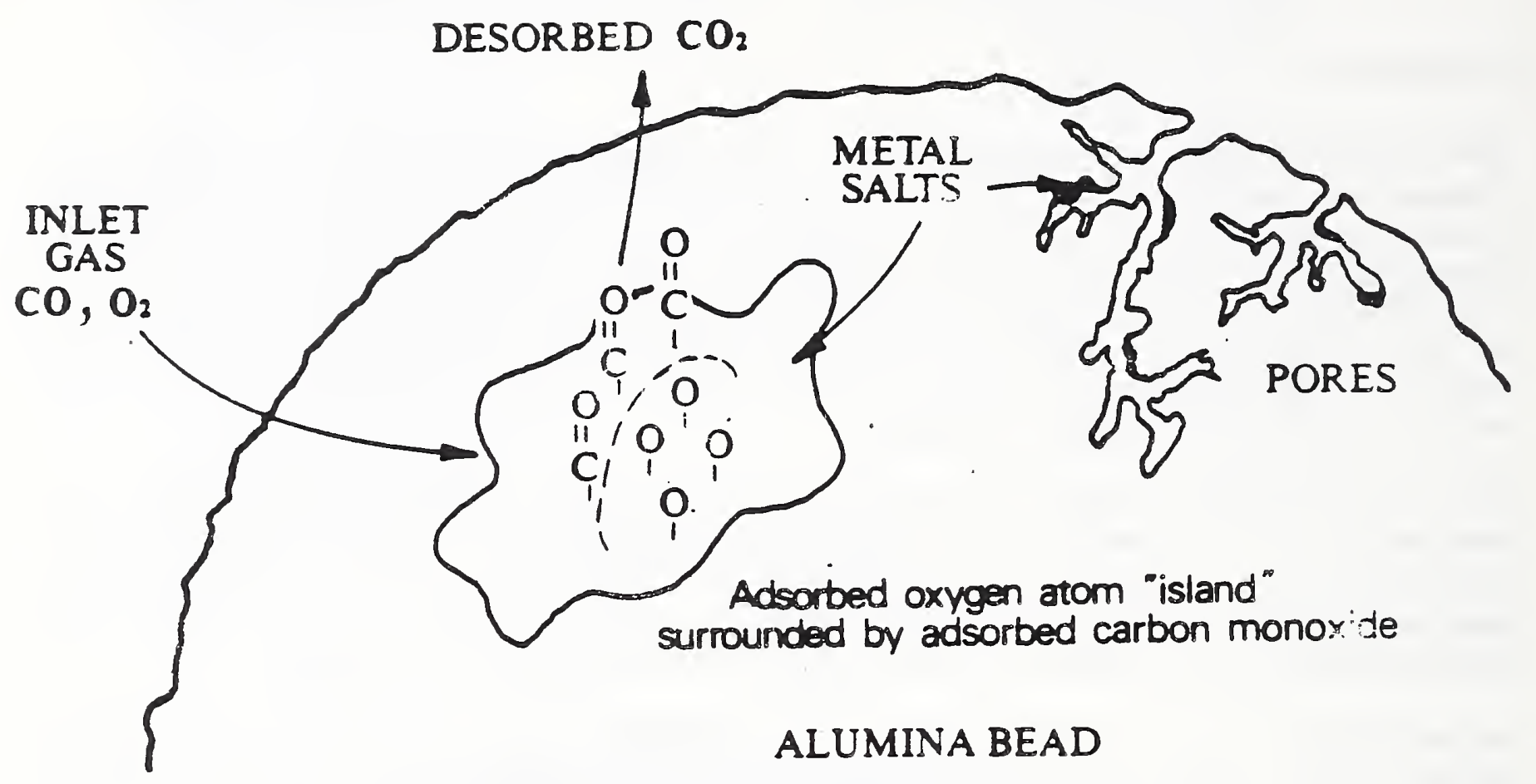

Figure 1. Diagram of Catalytic CO Oxidation Mechanism

\section{LTC ACTIVATED COMPLEX}

Several simple tests imply the formation of a palladium-cooper complex, probably stabilized by surrounding anions. This complex is the active species which is responsible for catalytic rates of $\mathrm{CO}$ oxidation to $\mathrm{CO}_{2}$.

A "catalyst" made with only the palladium salt--all else equal--was able to achieve 50 percent Co removal under standard tube test conditions. Likewise, a "catalyst" containing the two copper salts gave on ly 15 to 4 percent CO removal. The LTC combination of these three salts consistently removes greater than 85 percent $\mathrm{CO}$.

A series of tests using stepwise additions of the metal salts constituents in various sequence (same final composition) clearly shows the best co removals occur with the simultaneous deposit of the LTC composition.

SPECIFICATIONS FOR PERFORMANCE TESTS

Tube Test for Catalytic Activity

A one gram sample of LTC catalyst is packed in a glass tube to minimize bypass and exposed to feed gas flow of known concentration in a single contaminant. Relative humidity in the feed stream may be varied from 10 to 
90 percent, while temperature is unregulated ambient. Typical test conditions are 50 parts per million of carbon monoxide and 60 percent relative humidity at a flow rate of $500 \mathrm{cc} / \mathrm{minute}$. The difference in $c 0$ concentration between feed gas passed over untreated substrate alumina beads versus over the LTC catalyst measures activity, which can be monitored over time. Equilibrium removal rates are the percentage of pollutant concentration lost after 6 hours. The standard deviation of 8 repetitive test results was a satisfying 2.1 .

The actual amount of contaminant removed, of course, depends on physical parameters--the amount of ITC, the size and thickness of the catalyst bed, gas flow rate, length of testing, and initial concentration of contaminant co. Time of contact with LTC reflects the former variables and is calculated from:

$$
\ln \left(C_{0} / C_{t}\right)=R \cdot t_{c}
$$

Assuming a first-order reaction rate (oxidation of $\mathrm{CO}$ to $\mathrm{CO}_{2}$ ), $\mathrm{C}_{t}$ is the contaminant concentration at some time $t, C_{0}$ is the initial concentration, $\mathbb{R}$ is the reaction rate constant, and $t_{c}$ is contact time. In reality, the reaction rate also varies with the amount of surface coverage of oxygen and carbon monoxide, therefore a first order reaction scheme is a considerable simplification. The reaction constant is characteristic of catalyst material, independent of test procedures. For example, an LTC catalyst with an equilibrium Co removal rate of 93 percent, has a calculated $k$ of about 12 seconds ${ }^{-1}$.

Room Test of Air Filtration Appliance

The second type of performance test measures contaminant removal in a sealed room, using the LTC catalyst filter in our air filtration appliance. Filters were designed to hold about 230 grams of LTC catalyst in a thin bed with minimal air flow by-pass. This test is conducted in a 1008 cubic foot stainless steel room with an initially known amount of gaseous contaminant. The rate of decay is subtracted from the performance of the appliance. Air flow through the app liance was $300 \mathrm{cfm}$ for the test duration of 2 hours. The standard deviation of 7 repetitive test results wa an excellent 1.4 .

\section{TUBE TEST PERFORMANCE}

Temperature Dependence

The temperature dependence of LTC performance has been reported previously [4], but a brief description is included for completeness. Figure 2 maps results of a series of tests for CO removal of LTC at various temperatures. Between room temperature and about $400^{\circ} \mathrm{C}$, Co oxidation ranges between 90 and 100 percent. Below about $65^{\circ} \mathrm{F}$, the reaction rate drops off sharply, probably as a result of increased dissipation of the heat of reaction.

In contrast, the physical adsorption of $\mathrm{NO}_{2}$ decreases as the temperature increases. Near 100 percent adsorption occurs between $-50^{\circ}$ and $25^{\circ} \mathrm{C}$, gradually decreasing to 50 percent at $175^{\circ} \mathrm{C}$. 


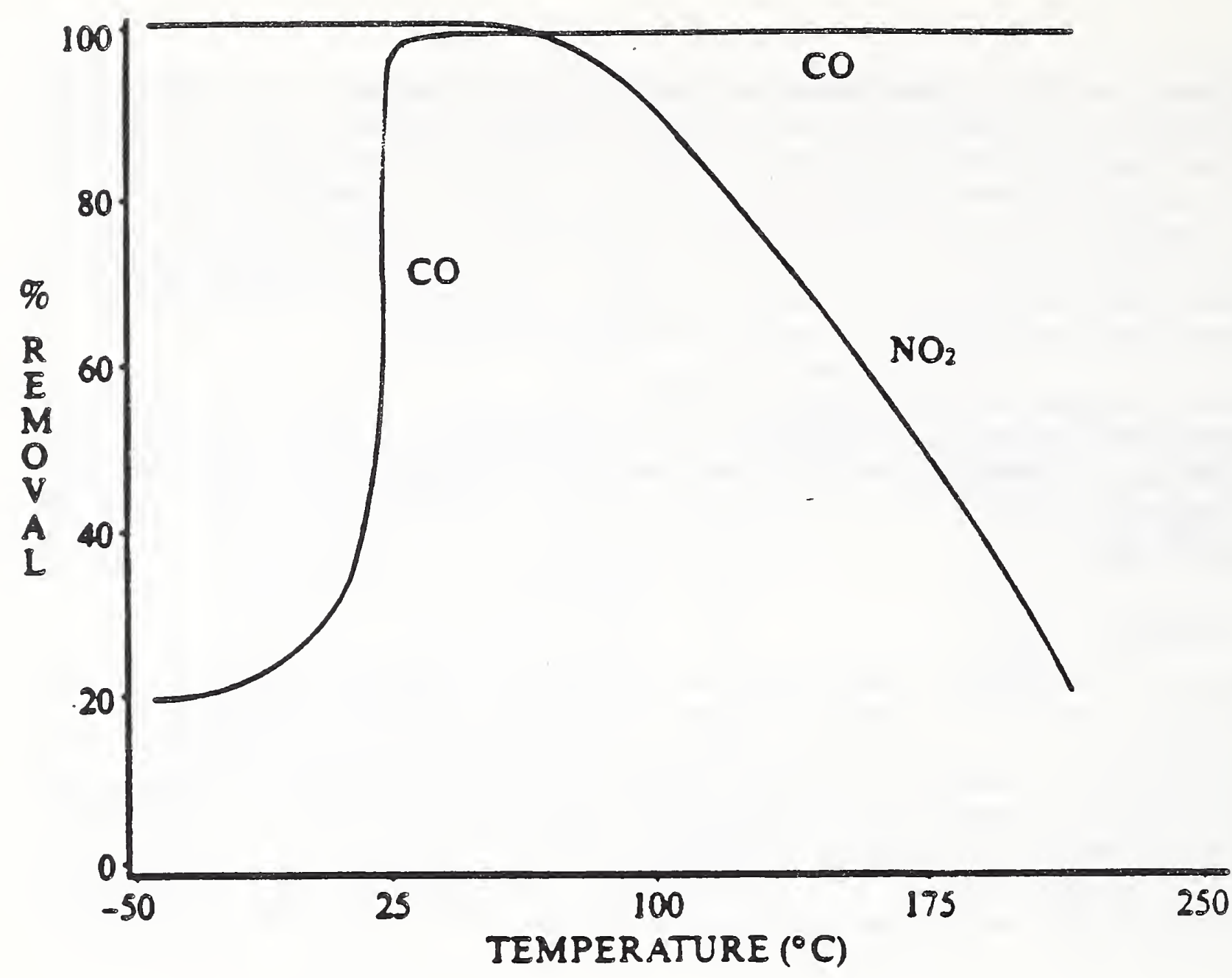

Figure 2. LTC Removal of $\mathrm{CO}$ and $\mathrm{NO}_{2}$ as a Function of Temperature Relative Humidity Dependence

The dependence of LTC activity on relative humidity was a lso reported previous ly [4], and is illustrated in Figure 3. Co oxidation is optimum between about 25 and 70 percent relative humidity. Inadequate water present inhibits the reaction at interfaces of adsorbed "is land oxygen" and surrounding $C O$ molecules. Too much moisture fills the micropores of the alumina, effectively "drowning" these active metal deposits.

The physical adsorption of $\mathrm{NO}_{2}$ is unaffected by humidity, presumably because most of the chemisorption is on the surface of the ITC/alumina bead and in the micropores. A common commercial material, Hopcalite, is severely poisoned by water vapor; it is ineffective about 10 percent relative humidity.

Contact Time

One of the physical parameters which strongly affects the LTC rate of co oxidation is the time that the contaminant is in contact with the catalyst. Table I shows data for contact time of 0.4 to 0.01 seconds achieved by varying the gas flow rate ( 5 to 0.5 /min) and/or the bed depth of LTC packed in the tube. All tests were conducted at room temperature and 60 percent relative humidity with 50 parts per million $\mathrm{CO}$. 


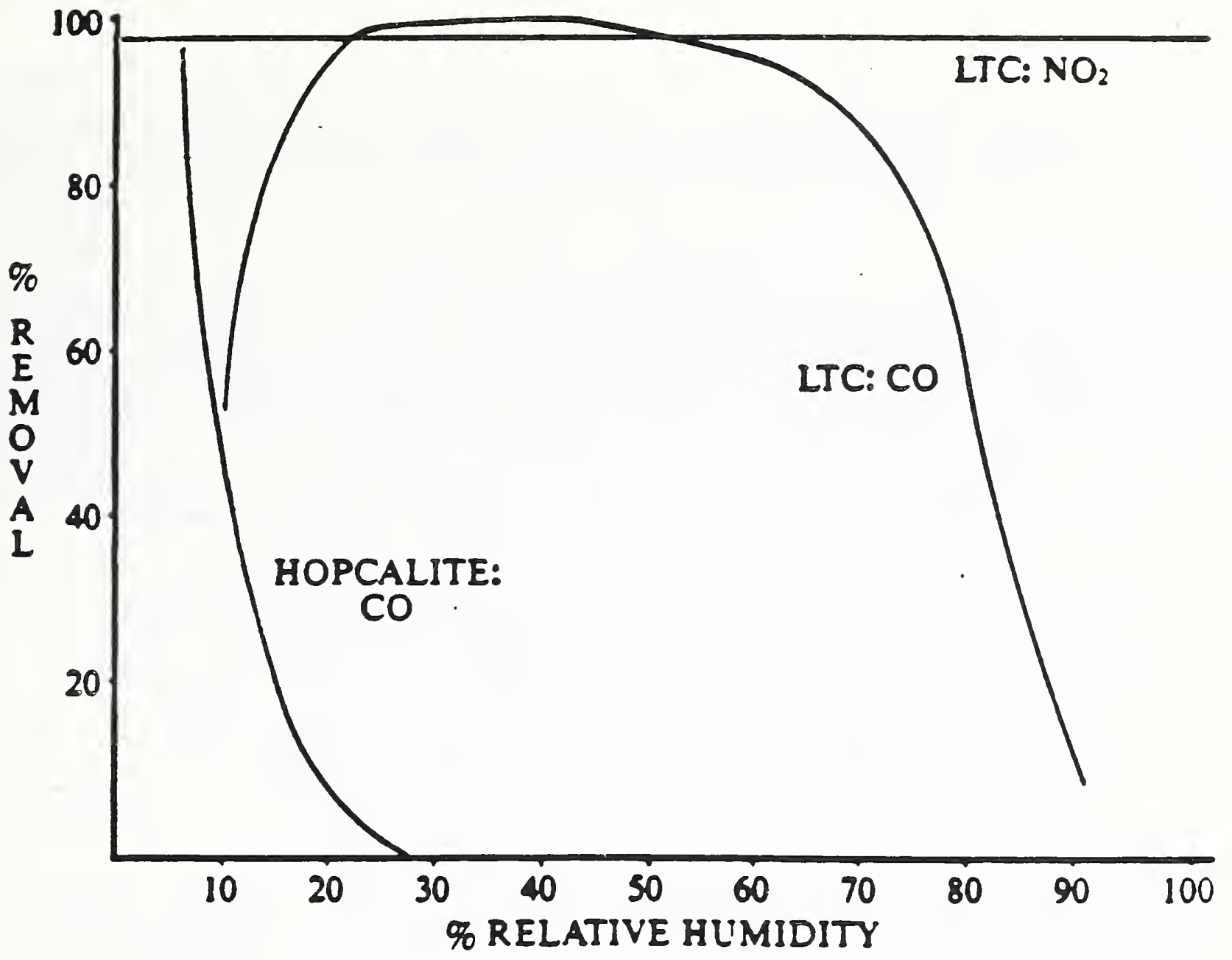

Figure 3. Catalyst Removals as a Function of Relative Humidity

TABLE I

Effect of Contact Time on CO Oxidation

Contact Time

(Sec.)

Bed Depth

(Inches)

0.4

0.2

0.1

0.08

0.05

0.025

0.01
1.0

0.5

0.5

0.4

0.25

0.25

0.25
\% Average

CO Remova 1

84

52

30

28

14

7

Although Equation 1 postulates a log-linear plot, the data fits a log-log curve--confirmed by regression analysis. The amount of surface coverage of the active LTC sites with $C O$ and oxygen molecules accounts in large part for the discrepancy, as we 11 as the fact that the oxygen-palladium bonds are weakened by the co-adsorption of $C 0$ molecules [6]. Thus, the reaction conforms more closely to the Langmuir-Hinshelwood mechanism-rather than a simple first order dependency [7].

ROOM TEST PERFORMANCE RESULTS

One present application of LTC catalyst is a portable air filtration appliance for home and office environments. The LTC beads are tightly packed into a thin filter to minimize pressure drop; in the product filters 
LTC and activated carbon are mixed 50:50. The Instapure ${ }^{R}$ ir Filtration System units used in this series of tests had LTC only (about 230 grams) and an air flow rate of $300 \mathrm{cfm}$. The contact time is thus approximately 0.02 second, or an order of magnitude less than that in the tube test. All of the following tests were conducted in a 1008 cubic foot sealed room at 40 percent relative humidity and ambient temperature. Initial contaminant concentrations are noted in each test, each of 2 hour duration. Figure 4 summarizes all of the results as adjusted for each species rate of decay. The Threshold Limit Value--Short Term Exposure Limit (STEL) are recommendations issued by the American Conference of Governmental Industrial Hygienists (1983). The STEL values cited are for a 15 minute time-weighted average which should not be exceeded during a work day.

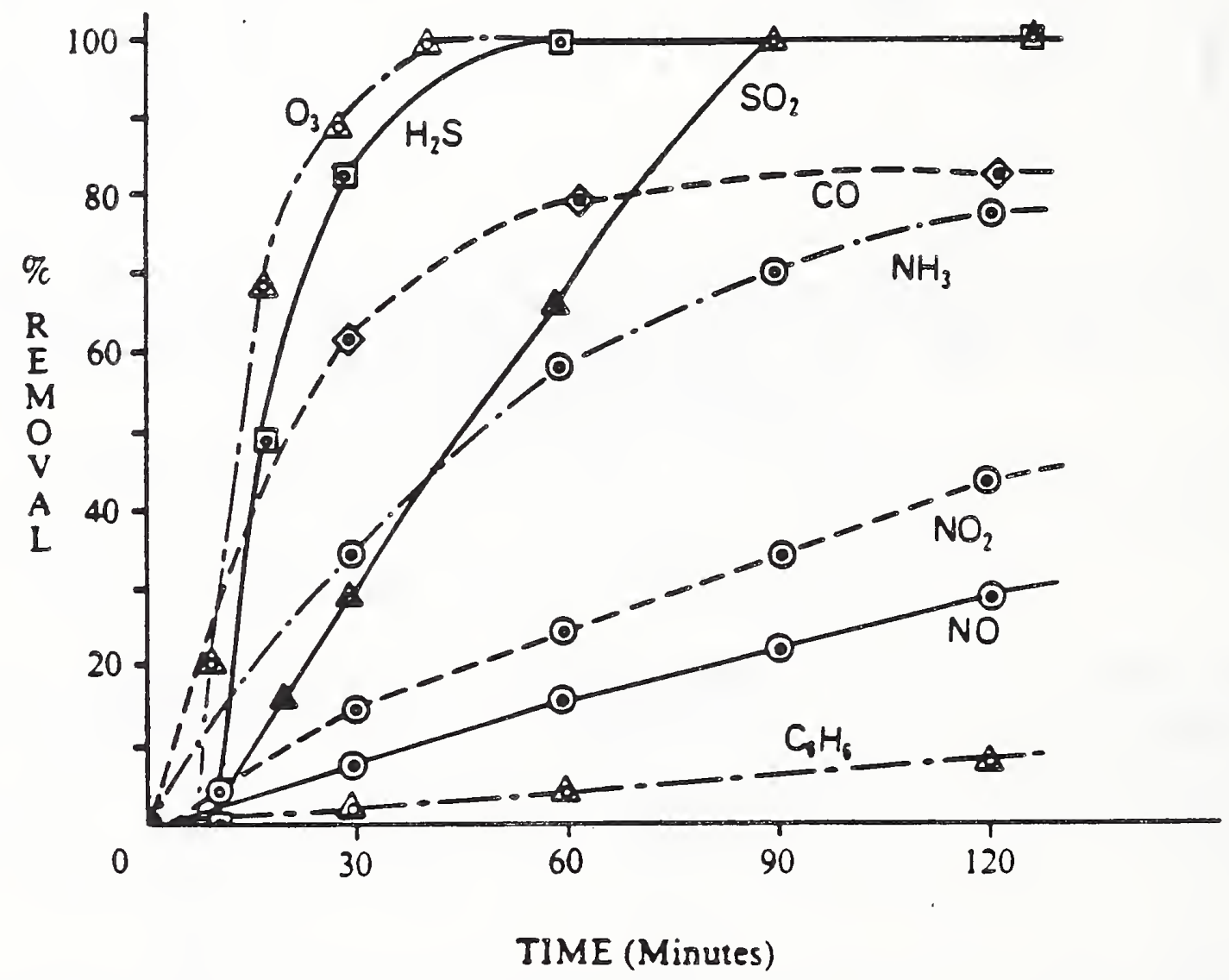

Figure 4. Room Test of LTC Filter for Various Contaminants

Carbon Monoxide

A filter using 50:50 LTC to activated carbon was tested, using 30 parts per million CO to begin. The STEL limit is 400 parts per million, but 30 parts per million corresponds to the lower limit of the "dangerous" category established by the EPA. After 2 hours running, 55 percent of the co was removed. Given the brief contact time, a per-pass filter efficiency of 14.6 percent may be calculated. An LTC filter removed 78 percent Co in 2 hours.

\section{Ozone}

An LTC-only filter in the appliance was run in the sealed room containing 164 parts per billion ozone (STEL limit is 300 parts per billion). After 
38 minutes, 99+ percent was removed. Other tests indicate that the very reactive ozone molecules are decomposed to oxygen by chemisorption and subsequent interaction on the alumina surface, as well as the ITC active sites.

\section{Nitric Oxide}

An LTC filter was exposed to 20 parts per million NO at $300 \mathrm{cfm}$ (STEL is 35 parts per million). After 80 minutes, the LTC had remove 20 percent, whereas the LTC/carbon filter was able to remove 40 percent of the initial concentration.

\section{Nitrogen Dioxide}

The LTC filter was exposed to 4 parts per million $\mathrm{NO}_{2}$ (STEL is 5 parts per million). Removal was 40 percent after 100 minutes versus 100 percent removal for the LTC/carbon filter. The $\mathrm{NO}_{2}$ molecules seem to be more tightly adsorbed to active carbon surfaces than to the LTC/alumina surfaces. As shown in Figure 2, increasing temperatures increase the rate of desorption (tube tests).

\section{Sulfur Dioxide}

An LTC filter in our appliance was run in the sealed room containing 5 parts per million $\mathrm{SO}_{2}$ (STEL is 5 parts per million). Removal of 100 percent was achieved after 90 minutes. Other tube tests [4] have shown that $\mathrm{SO}_{2}$ is chemisorbed by LTC active $\mathrm{Cu}$ (II) sites until saturation.

\section{Hydrogen Sulfide}

The ITC filter was subjected to 4 parts permillion $\mathrm{H}_{2} \mathrm{~S}$ (STEL is 15 parts per milition). After 60 minutes, about 100 percent of the $\mathrm{H}_{2} \mathrm{~S}$ was removed. Earlier tube tests indicate an irreversible reaction of $\mathrm{H}_{2} \mathrm{~S}$ with LTC which results in a gradual poisoning of the active sites. Apparenty, cooper sulfide is the reaction product [4].

Ammonia

An LTC filter in the air filtration appliance was run in an atmosphere containing 140 parts per million of ammonia. An STEL recommended limit of 35 parts per million is published. After 60 minutes, 58 percent was removed; 76 percent at the end of the 2 hour test. Subsequent, continuing test runs show that the catalyst is gradually saturated, with $\mathrm{NH}_{3}$ removal dropping to 6 percent after 6 hours.

Benzene

The LTC filter was exposed to 17 parts permillion of vapor in the test room (STEL limit is 25 parts per million). Only 8 percentwas removed after 2 hours, whereas the LTC/carbon filter was able to remove greater than 90 percent of the benzene concentration, even after each of five successive test runs. The benzene molecule is non-polar and readily adsorbed by the porous activated carbon. ITC is relatively unreactive to the benzene structure. 
Chemical components of both mainstream and sidestream tobacco smoke are present in air as particulate or aerosol and a gaseous phase. The major portion of particulate is tar, nicotine, and water; the majority of the gas phase is carbon dioxide, carbon monoxide and methane. Particulate can be removed mechanically from mainstream smoke by a paper filter (cigarettes). Table II 1 ists some of the higher concentration constituents of tobacco smoke along with their biological effects.

\section{TABLE II}

Some Cigarette Smoke Gaseous Constituents Mainstream Smoke: Unfiltered Cigarette

Gas Phase Components

Carbon Dioxide

Carbon Monoxide

Methane

Acetaldehyde

Acetone

Nitrogen Oxides

Isoprene

Hydrogen Cyanide

2 - Butanone

Acetonitrile

Toluene

Ammonia

Benzene

Acetylene

Dime thylnitrosamine

Nitrosopyrrolidine
Biological Activity

Wt/Cigarette

Toxic

Toxic

Toxic

Toxic

Toxic

Co-Carcinogen

Carcinogen

Carcinogen
$10-60 \mathrm{mg}$

$10-20 \mathrm{mg}$

$1.3 \mathrm{mg}$

$770 \mathrm{mg}$

$100-600 \mathrm{mg}$

$60-600 \mathrm{mg}$

$582 \mathrm{mg}$

$430 \mathrm{mg}$

$80-250 \mathrm{mg}$

$120 \mathrm{mg}$

180 mg

$80 \mathrm{mg}$

67 mg

$27 \mathrm{mg}$

$10-65 \mathrm{mg}$

10-35 mg

a - U.S. Dept. of Health, Education and Welfare, "The Hea 1 th Consequences of Smoking", 1981.

The most acute biological effect of tobacco smoke is suffered first from nicotine, and second $1 \mathrm{y}$ from carbon monoxide. The latter marked ly decreases the oxygen-carrying capacity of the blood and may impair the nervous system functions. Its effects range from inducing headaches and diziness to implication in heart attacks and strokes [8].

Room Test for CO From Cigarette Smoke

The test results for Co removal from sidestream cigarette smoke using the Instapure ${ }^{R}$ Air Filter $(A F-1)$ are shown in Figure 5. Two cigarettes were smoked every half-hour in a $1152 \mathrm{cubic}$ foot sealed room to gemerate the upper curve ending at 48 parts per million $C 0$ in 8 hours. The same procedure was followed while running the AF-l appliance, which gave the center curve ( 32 parts per million $C O$ in 8 hours). The lower curve is for a repeat of the second test with cigarette smoking terminated after 6 hours. The air filter removes 34 percent of the cumulative co concentration. 


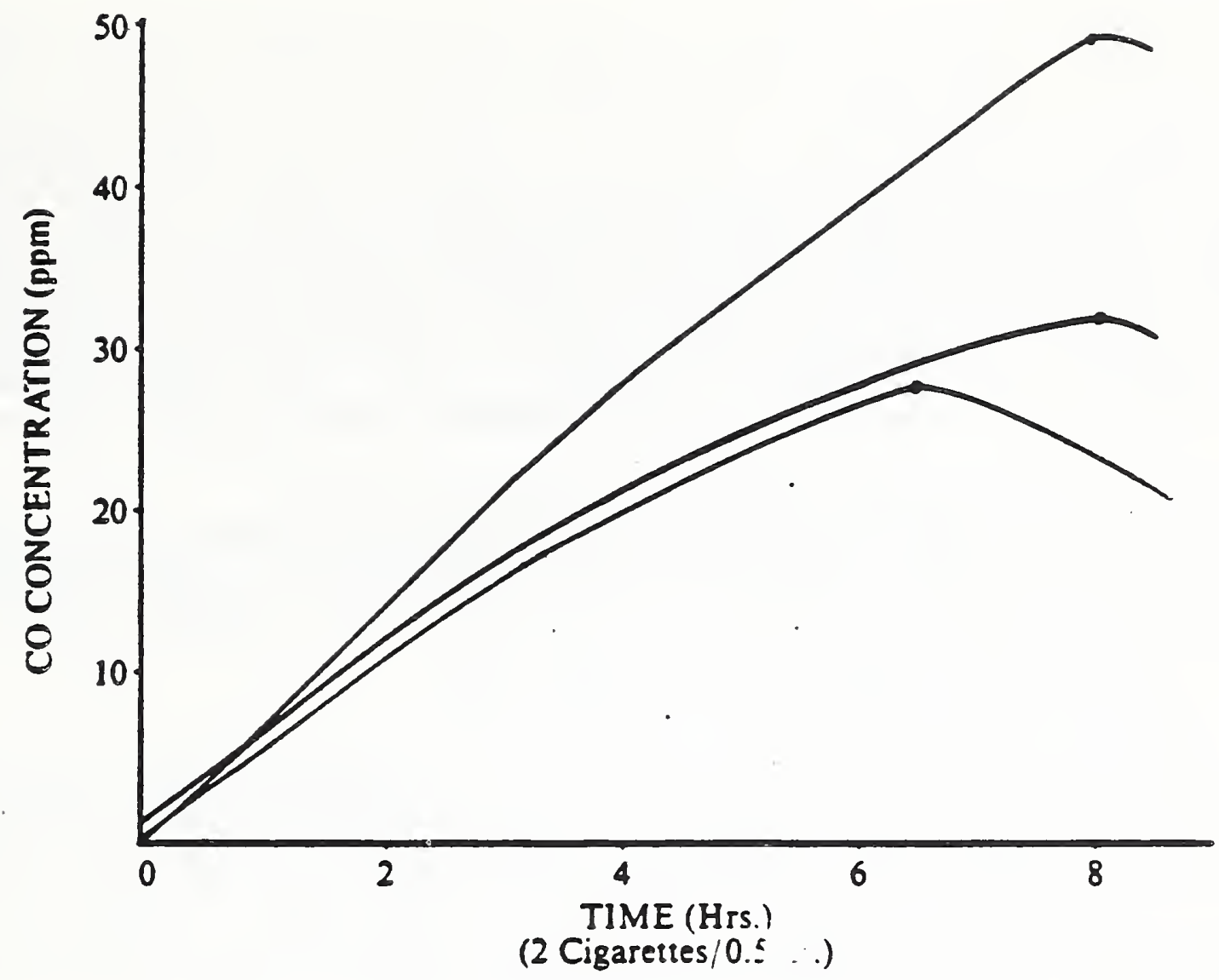

Figure 5. Room Tests of Product Filter for Co (Smoke) Removal Rate

Mainstream Smoking Test

A small filter containing 6 grams of LTC was attached to unfiltered cigarettes to test for removal of mainstream tobacco smoke components. The tests were conducted by an independent laboratory and are summarized in Figure 6. Oxidation of $\mathrm{CO}$ is dramatic--from 17,000 to 430 parts per million with the LTC filter. Referring to Table II, several other hazardous compounds are significantly reduced by use of LTC. Acetaldehyde and ammonia are diminished from 1,600 to 18 and 160 parts per million or less respectively, Likewise, methyl alcohol is mechanically removed; several constituents are below the detection level and therefore show no change.

\section{POLLUTANT}

Carbon monoxide

Hydrocarbons

Ammonia

Acetaldehyde

Methyl chloride

Acetone

Methyl alcohol

Methyl ethyl ketone

Hydrogen sulfide

\section{CIGARETTE (pDm)}

17,000

2,700

1,600

1,600

550

480

350

100

41

\section{LTC/CIGARETTE (ppm)}

430
600
160
$<\quad 18$
$<\quad 84$
$<\quad 7$
$<\quad 60$
$<\quad 6$
$<\quad 1$

Figure 6. LTC Removal of Contaminants in Cigarette Smoke 


\section{SUMMARY}

One of the important qualities of the LTC catalyst is the rapid oxidation of carbon monoxide to carbon dioxide under a wide variety of conditions. The catalytic material is a palladium-copper activated complex which reacts with various contaminant molecules through a continuous oxidation/reduction cycle. The alumina substrate enhances LTC activity with its favorable surface chemistry and very high surface area. About 10 percent surface water is necessary to facilitate the oxidation of $C 0$. This reaction shows a log-log dependence on contact time, suggesting a Langmuir-Hinshelwood mechanism.

In the tube tests, LTC removed 90 to 100 percent of contaminating carbon monoxide in the temperature region of $20^{\circ}$ to $400^{\circ} \mathrm{C}$, and at ambient over a range of 25 to 65 percent relative humidity. In contrast, $\mathrm{NO}_{2}$ is chemisorbed by the LTC/alumina material-the amount strongly dependent on temperature increases but independent of humidity.

Performance tests in the Instapure ${ }^{R}$ Air Filtration appliance were done in a sealed room using $300 \mathrm{cfm}$ air flow rate. $\mathrm{CO}$ was 78 percent removed, $\mathrm{O}_{3} 100$ percent removed, $\mathrm{SO}_{2}$ and $\mathrm{H}_{2} \mathrm{~S} 100$ percent, $\mathrm{NH}_{3} 58$ percent, $\mathrm{NO}_{2} 40$ percent, and benzene 8 percent removed. Co from sidestream tobacco smoke vas 34 percent removed by the appliance. Hazardous components of mainstream tobacco smoke were effectively reduced using a small ancillary filter containing LTC.

The LTC catalyst has demonstrated excellent capability to remove an important variety of hazardous pollutant gases which are common factors to poor indoor air quality. The Instapure ${ }^{R}$ Air Filtration System incorporates the LTC catalyst in a 50:50 mixture with activated carbon to effectively remove particulate, odors, and hazardous gages at room temperature and humidities. The ability to remove hazardous gases is unique for the category of portable air filtration equipment. The wide variety of pollutant gases that LTC removes suggests the catalytic technology is adaptable to a considerable range of commercial and industrial applications.

Disclaimer: NBS does not endorse commercial products. Responsibility for the information included here rests solely with the manufacturer. The information is included here because it is deemed useful to the objectives of the symposium.

\section{REFERENCES}

1. Wallace, L. A., "Results From the First Three Seasons of the Team Study: Personal Exposures, Indoor-0utdoor Relationships, and Breath Levels of Toxic Air Pollutants Measured for 355 Persons in New Jersey." U.S. EPA Research Triangle Park, N.C., 78 th Annual APCA meeting, June 16-21, 1985 .

2. Ibid, Et A1. "Personal Exposure to Volatile Organic Compounds: Direct Measurement in Breathing-Zone Air, Drinking Water, Food, and Exhaled Breath," Environmental Research, volume 35, 1984. 
3. Ibid, Et Al. "Assessing Sources of Volatile Organic Compounds, in Homes, Building Materials, and Consumer Products," Conference on Characterization of Sources of Indoor Air Contaminants, Releigh, N.C., May 13-15, 1985 .

4. Collins, M. F. "Room Temperature Catalyst for Improved Indoor Air Quality," Transactions of the Air Pollution Control Association, International Specialty Conference-Indoor Air Quality in Cold Climates: Pollutant Abatement Research, Ottawa, April 29 to May 1, 1985.

5. C. Thomas, Catalytic Process and Proven Catalyst, Academic Press, 1970.

6. Gasser, R. P. H., An Introduction to Chemisorption and Catalysis by Materials, Oxford University Press: 1985, Pp. 220-224.

7. Ibid, pp. 249-252.

8. "Smoking and Health: A report of the Surgeon General," U.S. Dept. of Health, Education, and Welfare, Part 15 , Biological Influences on Cigarette Smoking, 1981 . 


\title{
EFFECTS OF RELATIVE HUMIDITY ON ADSORPTION OF CONTAMINANTS ON ACTIVATED CARBON
}

\author{
Dwight W. Underhill, Corresponding Author \\ Graduate School of Public Health \\ University of Pittsburgh \\ 130 DeSoto Street \\ Pittsburgh, PA 15261
}

Gina Micarel1i and Maria Javorsky

Industrial Health Foundation, Inc.

34 Penn Circle West

Pittsburgh, PA 15206

\section{SUMMARY}

The Dubinin-Radushkevich potential theory was extended to include a term giving the effects. of relative humidity on the adsorption coefficient. This extended equation permits the adsorptive capacity of the activated charcoal in a respirator cartridge to be estimated for any combination of temperature, relative humidity, and concentration of contaminant. Application of this theory to previously published data of Werner showed a good correlation between theory and experiment. This equation is consistent with the experimental observations that 1 ) below a certain value, the relative humidity has little effect on the adsorption coefficient and 2) the effect of relative humidity, if observed, is more severe for lower than for higher concentrations of contaminant.

Because the uptake of water by activated charcoal increases rapidly as the relative humidity increases above $40 \%$, the performance of a respirator cartridge in humid atmospheres may be quite unsatisfactory. A worker may be at risk if he or she uses, in a highly humid environment, a respirator cartridge tested on ly 1) under dry conditions, or 2) on ly for a short period of time at a high relative humidity, or 3 ) for some other compound at a high relative humidity. The importance of this problem is compounded by the fact that the interfering compound, water vapor, is colorless, odorless, and usually present at very high concentrations.

In this paper we will develop from ideas of Polanyi, Dubinin, and Manes, a simple mathematical mode 1 for the effect of relative humidity on water insoluble compounds. We will then examine the predictions of this theoretical mode 1 and its accuracy when applied to previous 1 y published data. We also give data on the adsorption of water-immiscible solvents by a simulated respirator cartridge operating in an atmosphere of $100 \%$ relative humidity.

\section{The Polanyi Potential Theory}

In this study the uptake of mixtures of water vapor plus the vapors of an organic vapor, will be approached starting with the broad viewpoint of the Polanyi potential theory [1]. This theory is already known to be useful in predicting the adsorption of binary vapor mixtures [2, 3], including the uptake of binary mixtures of water vapor and organic vapors [4]. 
The basic concept of the Polanyi potential theory is that an adsorbent can be characterized by a series of force fields over the microporous surfaces of the adsorbent (Fig. 1, from Smisek and Cerny [5]). These attractive forces, which compress the molecules of a gas, act from the surface to a finite distance in the surrounding space. Polanyi has described these forces of attraction by the adsorption potential, which is defined for a point near the surface of the adsorbent, as the work done by the attractive forces in bringing a molecule of a gas to that given point. The adsorption potential has its maximum value at the surface of the adsorbent and it decreases to zero at some 1 imiting distance. The broken 1 ines shown in Fig. 1 represent planes of equal adsorption potential.

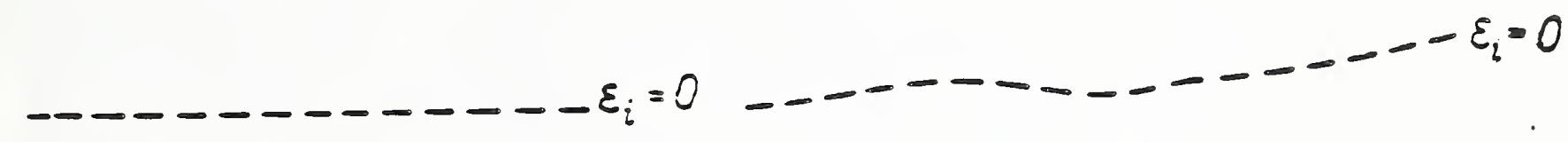

$$
\begin{aligned}
& ----------\varepsilon_{\downarrow} \\
& ---1---1--\hat{e}_{3}
\end{aligned}
$$

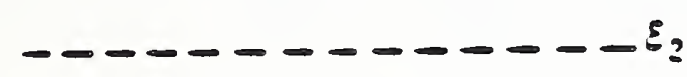

$$
\begin{aligned}
& ----\cdots,---\varepsilon_{1}
\end{aligned}
$$

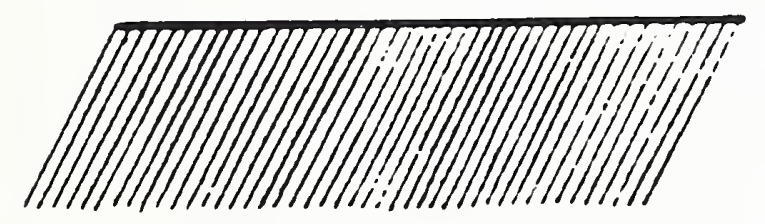

$$
\begin{aligned}
& \text { a) }
\end{aligned}
$$

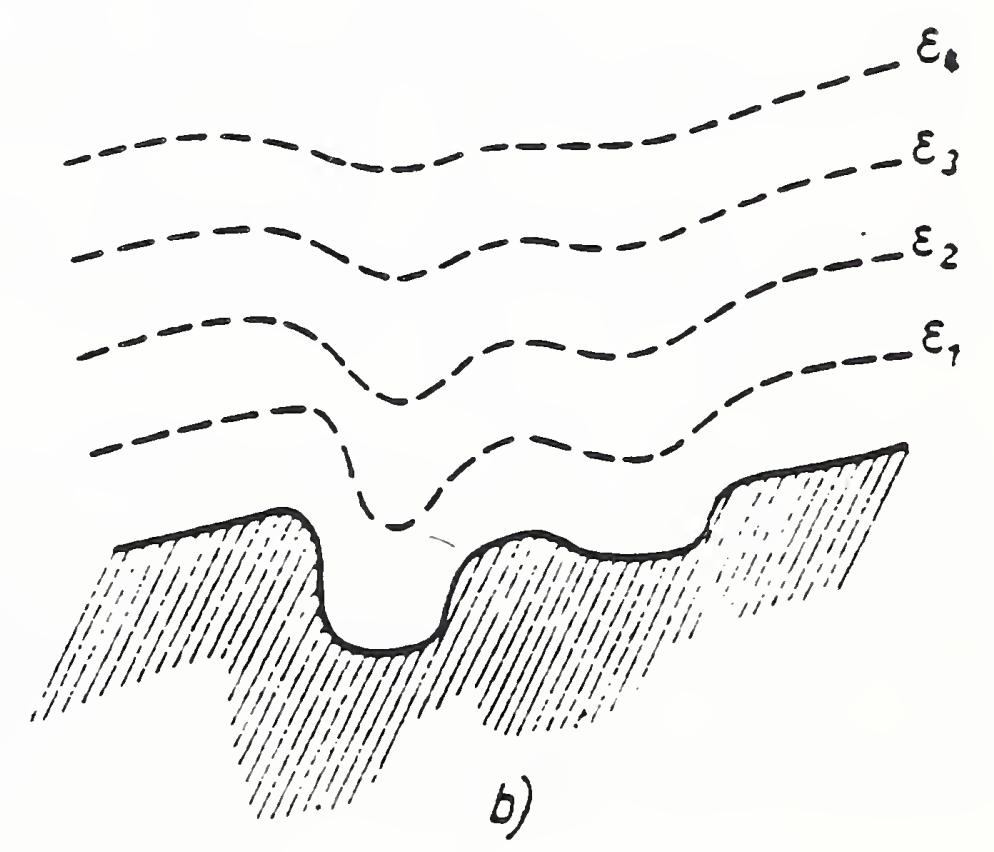

Fig. 1 Schematic representation of the force field at the surface of solid adsorbents according to the potential theory a) Idealized case of a plane homogeneous surface, b) Real surface from Smisek and Cerny [5].

Because the adsorption potential increases as the distance from the surface decreases, the density of the compressed adsorbate is not constant throughout the adsorption space. The adsorption potential may be defined in terms of, the isothermal work of compression per unit volume of adsorbed absorbate:

$$
=R T / V \ln \left(P_{0} / P\right)
$$

where: $T$ = absolute temperature, $\mathrm{OK}$

$$
\begin{aligned}
& \mathrm{R}=\text { ideal gas constant ( } 8.3143 \text { Joule/Mole/ } \mathrm{K}) \\
& \mathrm{V}=\text { molar volume, cc/mole } \\
& \mathrm{P}=\text { partial pressure of adsorbate, atm }
\end{aligned}
$$


$P_{0}=$ partial pressure of compressed absorbate on the given equipotential surface, atm

In the adsorption of vapors, if the isothermal work of compression causes $P_{0}$, the partial pressure of adsorbate on a given equipotential surface, to be greater than $P_{S}$, the vapor pressure of the pure adsorbate at the temperature of adsorption, then the adsorbate will be compressed to form a liquid phase held within this equipotential plane.

For the adsorption of vapors, it is usually a sufficiently accurate assumption to associate all of the adsorption that is observed with this liquefaction. This volume is calculated with the assumption that the adsorbate has the density of the saturated liquid phase. The relationship between the volume of adsorbate enclosed in the adsorption space and the adsorption potential is described by a characteristic curve. On a characteristic curve (so named because it should be the same for a given vapor and adsorbent at all temperatures below the critical temperature) the volume $\left(V_{t}\right)$ of an adsorbed film is expressed as a function of $R T / V$ $\ln \left(P_{s} / P\right)$. This characteristic curve takes the place of the more familiar plot of the adsorption isotherm, which gives the amount adsorbed as a function of pressure at a constant temperature.

Potential adsorption theories can be extended to given one characteristic curve for a wide number of compounds if provisions are made for the differences in the polarizability of the compounds under study. See for example Sansone and Jonas [6].

One of the limitations of the Polanyi potential theory is that it does not provide an analytical expression for the adsorption isotherm. Such equations must be found experimentally. Grant et a1. [4] in their study of adsorption of organic compounds on activated charcoal used the following expressions to characterize the Polanyi potential curve. For one activated carbon (30/100 mesh BPL activated carbon Audit Sample 104), they derive a fifth order polynomial to determine the adsorption space from the adsorption potential. And to calculate the inverse, i.e., the adsorption potential from the adsorbate volume, they use an eighth order polynomial.

\section{The Dubinin-Radushkevich Mode 1}

Dubinin and Raduskkevich [7] noted that in many cases it is not necessary to use high order polynomials to describe the adsorption potential. Very often the relationship between the adsorption potential and the adsorbed adsorbate is Gaussian:

$$
\mathrm{w}_{\mathrm{t}}=\mathrm{w}_{\mathrm{O}} \exp \left[-\mathrm{k}^{2}\right]
$$

where: $\quad W_{t}=$ weight of adsorbed adsorbate/gm adsorbent

$W_{0}=$ total (limiting) volume of the adsorption space

= density of liquid adsorbate at temperature of adsorption

$k=$ constant

Golovoy and Braslaw [8], working at Ford Motor Company, have demonstrated the high accuracy possible with the Dubinin-Radushkevich equation. In their work, a mean error of $1.9 \%$ was found when the Dubinin-Radushkevich equation was used to predict the adsorption coefficients of 14 solvents. 
Given the high accuracy possible with the use of the Dubinin-Radushkevich isotherm, the task that we have set for ourselves is to modify this equation so that it can be applied to the adsorption of water-immiscible solvents. Before this is done, we will examine how Manes has developed a graphical procedure using the Polanyi potential theory to describe the effect of adsorbed water on water-immiscible solvents.

\section{Manes Treatment of the Polanyi Potential Theory}

Manes [3] and Manes and Greenbank [9] have shown how, by simple geometric constructions, the Polanyi adsorption potential theory can be extended to the adsorption of water-immiscible adsorbates in the presence of water vapor. The basic concepts are shown graphically in Fig. 2, which describes the effect of relative humidity on the adsorption of butane from air. In Fig. 2, the curve on the far right represents the Polanyi potential curve for butane alone, and the curve marked $\mathrm{H}_{2} \mathrm{O}$ represents the Polanyi potential curve for water vapor. In the presence of water vapor at $100 \%$ relative humidity, the Polanyi potential curve for butane is shifted to the left by an amount that is equal to the potential energy for the adsorption of the water vapor. The lower set of horizontal arrows shows how this shift is made in order to determine the adsorption of butane from air saturated with water vapor.

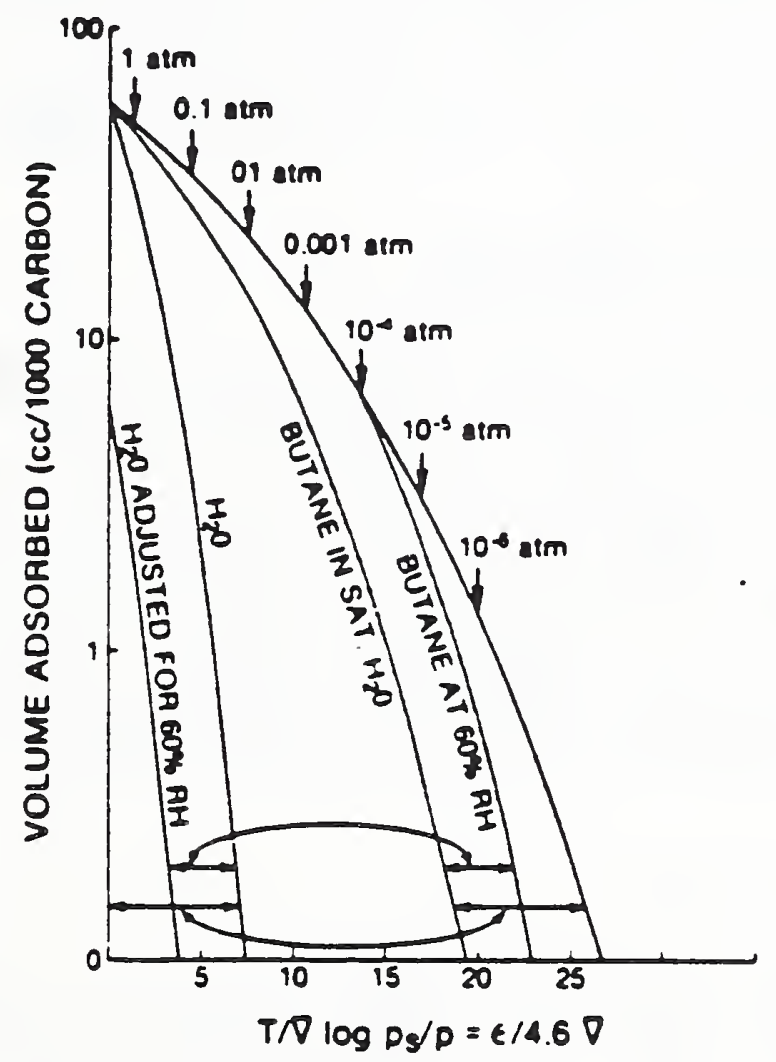

Fig. 2 Illustrative graphic calculation for adsorption of butane and water and $50 \%$ and $100 \%$ relative humidity. From Manes [3]

If the air is on ly partially saturated with water vapor, the polanyi potential for adsorption in the presence of water vapor is shifted to the left by an amount determined by the free energy lost by the water vapor 
because of its partial saturation. This shift is proportional to $R T \cdot 1 n(R H / 100)$, where $R$ and $T$ have their usual values, and $R H$ is the relative humidity. In Fig. 2, the upper set of horizontal arrows shows the determination of the adsorption of butane from air which was $60 \%$ saturated with water vapor. The result of this shift is an increase in the quantity of adsorbate over what would be seen in the presence of air completely saturated with water vapor.

From Fig. 2, other effects of the combined concentrations of water vapor and organic adsorbate on the adsorption of the organic adsorbate may be determined. At the higher adsorption potentials (i.e. at low concentrations of the organic adsorbate), the filled pore volume changes very rapidly with small changes in the adsorption potential, and thus it would be expected that at low concentrations of organic adsorbate, the relative humidity would have its strongest effect.

A second prediction from Manes correlation is that at relative humidities less than $100 \%$ and at a sufficiently high concentration of an organic vapor, the relative humidity causes a negligible reduction in the adsorption coefficient of the organic component. The physical explanation for this effect is that at less than $100 \%$ relative humidity, the pores are only partially filled with water. At a certain vapor pressure of the organic adsorbate, the organic adsorbate will fill the pores beyond the level occupied by the water, displacing a 11 the water, and at this concentration (as well at higher concentrations) of organic adsorbate, the presence of water vapor has no effect on the adsorption of the organic vapor. This effect is seen in Fig. 2, in which at pressures of butane over $10^{-4}$ atm, and at a relative humidity of $60 \%$, the quantity of adsorbed butane is the same as from dry air.

\section{Calculation of the Ad sorption Coefficient}

Rather than depend on the graphical analysis, we develop here an equation which permits a rapid calculation of the effect of water vapor on the adsorption of water immiscible organic compounds. We use three assumptions implicit in the geometrical analysis that Manes developed from the Polanyi diagram, for estimating the effect of moisture on the adsorption of water immiscible compounds. The first is that if, from dry air the potential free energy required to fill a micropore with organic vapor is, then the potential energy required in the presence of completely saturated air is some multiple of the free energy required in the absence of water vapor, i.e.

$$
100 \% \mathrm{RH}=\left(1+\mathrm{k}_{1}\right) \text { dry air }
$$

The second assumption is that the free energy required to fill a micropore with condensed organic vapor decreases linearly with a decrease in the free energy of the water vapor. Thus, to calculate the adsorption of organic vapors from air which is partially saturated with water vapor, a correction factor of proportional to $\mathrm{RT} / \mathrm{V}_{\mathrm{h}}{ }^{\circ} \mathrm{ln}(\mathrm{RH} / 100)$, where $\mathrm{V}_{\mathrm{h}}$ is the molar volume for water and $\mathrm{RH}$ is the percent relative humidity, should be used. Thus the potential free energy required for pore filling by a water immiscible organic compound is:

$$
=\left(1+k_{1}\right) R T / V \cdot \ln \left(P_{s} / P\right)+k_{2} R T / V_{h} \cdot \ln (R H / 100)
$$


where: $k_{1}$ =factor defined in Eq. 2 to describe the change in the adsorption potential of a water immiscible compound brought about by replacing the air with water in the micropores

$\mathrm{K}_{2}=$ factor giving the change in the adsorption potential of a water immiscible compound as a function of the relative humidity

$V_{h}=$ molar volume of water, $\left(55.5 \mathrm{~cm}^{3} / \mathrm{gm}\right)$

$R, T, V, P$, and $P_{S}$ are as defined previously.

However if $k_{2} R T / \nabla_{h} \cdot \ln (R H)>k_{1} R T / V \cdot l n\left(P_{s} / P\right)$, then the organic adsorbate can displace all the water from the micropores and the adsorption potential for the organic solvent is given by Eq. I, which does not contain any factors for the effect of coadsorbed water, as it is presumed that under this condition any competing coadsorbed water has been completely displaced from the adsorbent.

After the adsorption potential for the organic solvent has been determined, using either Eq. 1 or 4, whichever is appropriate, the uptake of organic adsorbate is calculated from Eq. 2 .

\section{Experimental Studies}

Realistic tests on the effect of relative humidity on the adsorption of a water immiscible organic compound were reported recently by Werner [10], who challenged small beds (containing 37.5 gms) of activated charcoal to input concentrations of 300 to $1300 \mathrm{mg} / \mathrm{m}^{3}$ of $\mathrm{trichloroethylene}$ (TCE) at relative humidities ranging from 5 to $85 \%$. Werner found that the lower the concentration of adsorbate (trichloroethylene), the stronger the effect of relative humidity. In Werner's paper this effect was shown in a series of empirical curves in which the uptake was plotted against the square of the logarithm of the concentration. Our object in reanalyzing these data is to find a quantitative expression of these results. To do this we reexamined Werner's data in terms of the procedure we developed above.

Table I gives Werner's measurements of the uptake of trichloroethylene on activated carbon, as a function of the temperature and relative humidity. The first step in this analysis, to determine the appropriate values for $k$ and $W_{0}$, was based on Werner's measurements of the adsorption of trichloroethylene at $5 \%$ relative humidity. It was assumed that at this relative humidity, the experimental data were equivalent to the adsorption on dry charcoal. These two parameters, the usual parameters in the Dubinin-Radushkevich equation, were then used in conjunction with trial and error tests to find the best values for $k_{1}$ and $k_{2}$ to fit Eq. 4 to the measurements of adsorption of trichloroethylene at higher relative humidities. Thus the effect of relative humidity was determined with only two adjustable parameters, one of which, $k_{1}$, gives the maximum effect of adsorbed water on the adsorption of the immiscible compound, and the other parameters, $k_{2}$, describes the increase in the adsorption of the immiscible compound as the partial pressure of the water vapor is reduced. For $k_{1}$ and $k_{2}$ the values found by a trail and error procedure were 0.87 and 4.75 , respectively. When these values were used with Eqs. 4 and 2 , the mean error between our calculated values and Werner's experimental values is $10.5 \%$, which is in the same range as the experimental error in Werner's measurements. These two equations give a quantitative way of representing 
the basic findings of Werner, i.e. 1) below a certain value, the relative humidity has little effect on the adsorption coefficient and 2) the effect of relative humidity, if observed, is more severe for lower than for higher concentrations of contaminant.

Table I

Predicted vs. Theoretical Uptake of Trichloroethylene in the Presence of Water Vapor

\begin{tabular}{|c|c|c|c|c|c|c|c|c|}
\hline \multirow[b]{2}{*}{ R.H. } & \multicolumn{2}{|c|}{$\begin{array}{l}\text { Uptake at } \\
1300 \mathrm{mg} / \mathrm{m}^{3}\end{array}$} & \multicolumn{2}{|c|}{$\begin{array}{l}\text { Uptake at } \\
1000 \mathrm{mg} / \mathrm{m}^{3}\end{array}$} & \multicolumn{2}{|c|}{$\begin{array}{l}\text { Uptake at } \\
600 \mathrm{mg} / \mathrm{m}^{3}\end{array}$} & \multicolumn{2}{|c|}{$\begin{array}{l}\text { Uptake at } \\
300 \mathrm{mg} / \mathrm{m}^{3}\end{array}$} \\
\hline & Calc. & Meas. & Calc. & Meas. & Calc. & Meas. & $\mathrm{Calc}$. & Meas. \\
\hline $5 \%$ & 0.429 & 0.434 & 0.397 & 0.399 & 0.346 & 0.334 & 0.281 & 0.286 \\
\hline $25 \%$ & 0.434 & 0.431 & 0.434 & 0.370 & 0.250 & 0.262 & 0.114 & 0.121 \\
\hline $50 \%$ & 0.399 & 0.403 & 0.387 & 0.342 & 0.209 & 0.218 & 0.091 & 0.098 \\
\hline $65 \%$ & 0.334 & 0.320 & 0.297 & 0.284 & 0.147 & 0.160 & 0.059 & 0.054 \\
\hline $85 \%$ & 0.286 & .0 .257 & 0.190 & 0.180 & 0.084 & 0.114 & 0.030 & 0.027 \\
\hline
\end{tabular}

In using Eqs. 2 and 4 to make the calculations given in Table I, the following parameters were used:

$$
\begin{aligned}
& =1.455 \mathrm{gm} / \mathrm{cm}^{3} \\
\mathrm{P}_{s} & =500,500 \mathrm{mg} / \mathrm{M}^{3} \\
\mathrm{~W}_{0} & =0.62 \mathrm{~cm}^{3} / \mathrm{gm}^{6} \\
\mathrm{k} & =2.89 \times 10^{-5} \mathrm{~cm}^{6} / \text { Joule } \mathrm{e}^{2} \\
\mathrm{k}_{1} & =0.87 \text { (dimensionles } \mathrm{s}) \\
\mathrm{k}_{2} & =4.76 \text { (dimensionless) }
\end{aligned}
$$

Experimental data from Werner [10]

We were fortunate in having an extensive set of data, such as Werner's, through which our equation for the effect of relative humidity on the adsorption coefficient can be tested. But Werner's data extends to relative humidities no higher than $85 \%$, and it is important to know the adsorption coefficient and mass transfer efficiency of respirator cartridges at relative humidities as high as $100 \%$. Performance of simulated respirator cartridges at higher relative humidities will be examined next.

Table II shows the results from a different study [11] in which xylene was adsorbed from air saturated with water vapor. The purpose of this work was to examine the performance of simulated respirator cartridges in air totally saturated with water vapor, and therefore the experimental parameters, $i . e .$, the mass of charcoal ( $40 \mathrm{gms}$ ), the flow of air (20 $1 \mathrm{pm})$, and the concentration of contaminant (ranging from 250 to 1000 ppm) represented realistic parameters for estimating the in-use performance of a respirator cartridge at a high relative humidity. The adsorbent, 8/16 mesh Barnebey-Cheney Grade 非177, activated carbon having an activity of $60 \%$, was dried overnight at $145-155^{\circ} \mathrm{C}$ before being tested. The adsorption 
coefficient, in liters/gm, was calculated from the breakthrough curves using a dimensionalized version of Eq. 16-47 of the Chemical Engineers' Handbook [12]. The "Use Time" in Table II represents the time before the effluent concentration of xylene from the adsorption bed reached $5 \%$ of the input concentration. It is seen from the "Use Times" in Table II that the simulated respirator offered protection against xylene fro an appreciable time, even though the carrier gas, air, had been saturated with water vapor. For xylene concentrations of 500 ppm and lower, effective protection (based on the criterion of less than $5 \%$ breakthrough) would be expected to be longer than a standard workday of 8 hours ( 480 minutes).

Table II

Effect of Concentration on Adsorption of Xylene by Predried Charcoal from Air Saturated with Water Vapor (from Micarelli [11])

\begin{tabular}{cccccc}
$\begin{array}{c}\text { Input } \\
\text { Concentration }\end{array}$ & $\begin{array}{c}\text { Run } \\
\text { 非 }\end{array}$ & $\begin{array}{c}\text { Adsorption } \\
\text { Coefficient } \\
\text { (1iter/gm) }\end{array}$ & $\begin{array}{c}\text { Theoretical } \\
\text { Plates }\end{array}$ & $\begin{array}{c}\text { Use } \\
\text { Time } \\
\text { (Mins) }\end{array}$ & $\begin{array}{c}\text { Percent } \\
\text { Saturation }\end{array}$ \\
\hline $\begin{array}{l}1000 \mathrm{ppm} \\
500 \mathrm{ppm}\end{array}$ & 34 & 202.5 & 37.0 & 204.4 & 50 \\
$250 \mathrm{ppm}$ & 30 & 460.0 & 46.6 & 527.0 & 57 \\
\hline
\end{tabular}

In the above table:

Use Time = Time (in minutes) for which breakthrough remained less than $5 \%$ of inflowing adsorbate.

$\%$ Saturation = Percent saturation of adsorbent at time of $5 \%$ breakthrough.

The salient point from these measurements is not the protection offered against xylene, per se, but rather that it is reasonable to expect that there may be a large number of compounds which like xylene are water immiscible and will show similar retention times. We next present data on the adsorption of chlorinated hydrocarbons, which are water immiscible, and further, because of the high polarizability of the chlorinated hydrocarbons, which are water immiscible, and further, because of the high polarizability of the chloride atom, these compounds would be expected to be strongly attracted by the van der Waals force to the micropores of the charcoal and therefore to strongly resist displacement by adsorbed water.

Table III gives results of Javorsky [13], from experiments in which she measured the performance of simulated respirator cartridges when challenged with vapors of chlorinated hydrocarbons in air at both high and low relative humidities. The test compounds were chlorobenzene, tetrachloroethylene, methyl chloroform, carbon tetrachloride, and chloroform. Other than the adsorbates (chlorinated hydrocarbons) and their concentration, the experimental conditions were unchanged from those selected by Micarelli. For each compound, two breakthrough curves were determined using air at $100 \%$ R.H. and a comparison breakthrough curve was made using air at a relative humidity of $25 \%$. From each breakthrough curve the adsorption coefficient, the "Use Time" ( $t$ ime of $5 \%$ breakthrough), and 
percent saturation, which is the percent of the adsorption capacity of the bed that was utilized at the time when the effluent concentration reached $5 \%$ of the input concentration. To an industrial hygienist, this work has special importance because chlorinated hydrocarbons are often used in processes, such as degreasing, where steam is also used and thus where exceptionally high relative humidities may be expected.

Table III

The Performance of Respirator Cartridges with the Input Concentration of Chlorinated Hydrocarbon Maintained at 750 ppm (from Javorsky [13])

\begin{tabular}{|c|c|c|c|c|c|c|}
\hline CMPD & $\begin{array}{c}\text { Run } \\
\text { 非 }\end{array}$ & $\mathrm{RH}$ & $\begin{array}{l}\text { Use } \\
\text { Time } \\
\text { (Min) }\end{array}$ & $\begin{array}{l}\text { Adsorption } \\
\text { Coefficient, } \\
k,(1 / g m)\end{array}$ & $\begin{array}{c}\text { Percent } \\
\text { Saturation }\end{array}$ & $\begin{array}{l}\text { Ratio } \\
\mathrm{k} \text { at } 10 \\
\mathrm{RH} \text { to } \\
25 \% \mathrm{R} \\
\end{array}$ \\
\hline$C B$ & 1 & $100 \%$ & 375 & 298.1 & 62 & \\
\hline$C B$ & 2 & $100 \%$ & 370 & 290.4 & 63 & 0.93 \\
\hline $\mathrm{CB}$ & 19 & $25 \%$ & 508 & 315.2 & 80 & \\
\hline $\mathrm{TC}$ & 36 & $100 \%$ & 315 & 247.2 & 75 & \\
\hline $\mathrm{TC}$ & 37 & $100 \%$ & 290 & 243.1 & 60 & 0.70 \\
\hline IC & 41 & $25 \%$ & 542 & 349.1 & 77 & \\
\hline MC & 43 & $100 \%$ & 290 & 216.5 & 66 & \\
\hline $\mathrm{MC}$ & 44 & $100 \%$ & 273 & 214.7 & 63 & 0.78 \\
\hline $\mathrm{MC}$ & 45 & $25 \%$ & 407 & 276.7 & 73 & \\
\hline CT & 25 & $100 \%$ & 183 & 124.3 & 74 & \\
\hline $\mathrm{CT}$ & 26 & $100 \%$ & 176 & 119.4 & 73 & 0.81 \\
\hline $\mathrm{CT}$ & 29 & $25 \%$ & 243 & 150.0 & 81 & \\
\hline $\mathrm{CF}$ & 5 & $100 \%$ & 123 & 86.3 & 71 & \\
\hline$C E$ & 6 & $100 \%$ & 120 & 85.7 & 70 & 0.71 \\
\hline $\mathrm{CF}$ & 20 & $25 \%$ & 182 & 119.8 & 76 & \\
\hline where: & $\begin{array}{l}\mathrm{CB}= \\
\mathrm{TC}= \\
\mathrm{MC}= \\
\mathrm{CT}= \\
\mathrm{CF}=\end{array}$ & $\begin{array}{l}=\text { Chlorob } \\
=\text { Tetrach } \\
=\text { Methyl } \\
=\text { Carbon } \\
=\text { Chrloro }\end{array}$ & $\begin{array}{l}\text { zene, } \\
\text { roethy } \\
\text { lorofor } \\
\text { trachlo } \\
\text { rm. }\end{array}$ & He, and & & \\
\hline
\end{tabular}

From Table III, it can be seen that the change from $25 \%$ R.H. to $100 \%$ R.H. reduced the adsorption coefficient, on the average by $21 \%$ and the percent saturation, on the average by $12 \%$. Both these factors affect the "Use Time", which, on the average, was reduced by $32 \%$. The important point to be learned from Table III is that both the adsorption coefficient and the mass transfer efficiency are reduced by high relative humidities, and that both factors must be considered in determining the performance of respirator cartridges at a high relative humidity.

Why does the relative humidity affect the mass transfer efficiency (as described here by the percent saturation at $5 \%$ breakthrough)? The answer 
lies in the pore structure of the adsorbent. In most carbonaceous adsorbents [14] there are usually two isolated pore varieties, adsorbing pores and transport pores, which vary in their properties. Adsorbing pores consist of micropores $(r<6-7 \mathrm{~A})$ and supermicropores $(6-7 \mathrm{~A}<\mathrm{r}<15-16 \mathrm{~A}$ ) whereas transport pores consist of mesopores (15-16<r<1000-2000 A) and macropores $(r>1000-2000 \mathrm{~A})(15)$. The overall controlling rate of adsorption is usually the rate of transport of the adsorbate through the transport pores to the adsorbing pores, where it becomes bound by the van der Waals force. The probable explanation for the reduced mass transfer efficiency at a relative humidity of $100 \%$, is that the transport pores had become partially, but not fully clogged by water under the test conditions. Had the transport pores become fully clogged with water, then much poorer performance would have been expected because the diffusion of organic compounds through water is about a thousand times slower than through air.

It is important to understand the exact cause of the loss of respirator performance. If it were the reduction in the adsorption coefficient that caused the major loss of efficiency at a high relative humidity, then the only way to design a respirator cartridge that will be effective for a longer time under the same conditions is to use a respirator cartridge with more adsorbent. But if, instead, it is the mass transfer efficiency that is primarily affected, than an alternative to the use of more charcoa 1 would be a redesign of the respirator to give a geometry conducive to a higher mass transfer efficiency. This study has shown that for the adsorption of chlorinated hydrocarbons at a high relative humidity, both factors appear to be important.

\section{Topics for Additional Study}

The calculations reported here apply only to the adsorption of waterimmiscible compounds. The extension of the Dubinin-Radushkevich theory to the adsorption of water-miscible solvents are solved theoretically by Bering and coworkers $[16,17]$, who rewrote the Dubinin-Radushkevich equation in terms of partial molar quantities of the competing solvents and then experimentally proved the validity of their extension. There is no reason as to why the equations developed by Bering et al. would not apply to the coadsorption of water and water-miscible solvents.

Recently there has been an increased interest in the performance of respirator cartridges at high relative humidities. For example at the 1986 American Industrial Hygiene Conference there were six papers giving additional data in this area [References $18,19,20,21,22,23$ ]. We hope that the general procedures described here will be of value in correlating data in this very active field.

\section{Acknowledgment}

This work was supported by NIOSH Grant 1 RO1 OHOl644-01.

\section{References}

1. Polanyi, M.: Adsorption of Gases (Vapors) by a Solid Nonvolatile Adsorbent. Verh. deut. phys. Ges.18:55 (1916). 
2. Jonas, L. A., Sansone, E. B., Farris, T. S.: Prediction of Activated Carbon Performance for Removal of Binary Vapors. Am. Ind. Hyg. Assoc. J. 44:716-719(1983.

3. Manes, Mo: Estimation of the Effects of Humidity on the Adsorption onto Activated Carbon of the Vapors of Water-Immiscible Organic Liquids. Fundam. Adsorpt. Proc. Eng. Found. Conf. P335-44 (1984).

4. Grant, Ro Jo, Joyce, R. So, and Urbanic, J. Eo: The Effect of Relative Humidity on the Adsorption of Water Immiscible Organic Vapors on Activated Carbon, Fundam. Adsorpt. Proc. Eng. Found. Conf. P219227. (1984).

5. Smisek, Mos and Cerny, S.: Active Carbon, Manufacture, Properties and Applications, P104, Elsevier Publishing Co., Amsterdam (1970).

6. Sansone, E. B., and Jonas, L. A.: Prediction of Activated Carbon Performance for Carcinogenic Vapors, Amer. Ind. Hyg. Assn. J.42:688691 (1981).

7. Dubinin, M. M., and Radushkevich, L. V.: Equation of the Characteristic Curve and Activated Charcoal, Doklady Akad. Nauk. S.S.S.R. 55:327-9 (1947).

8. Golovoy, A., and Braslaw, J.: Adsorption of Automotive Paint Solvents on Activated Carbon, J. Air Pollut. Control Assn. 31:861-5 (1981).

9. Manes, M. , and Greenbank, M॰: Adsorption of Multicomponent Liquids from Water onto Activated Carbon: Convenient Estimation of Methods; Advances in Chemistry Series, No. 202, Treatment of Water by Granular Activated Carbon, eds, M. J. McGuire and I. H. Suffet, Published by the American Chemical Society, Washington, D.C.

10. Werner, M. D.: The Effects of Relative Humidity on the Vapor Phase Adsorption of Trichloroethylene by Activated Charcoal, Am. Ind. Hyg. As soc. J. 46:585-90 (1985).

11. Micare11i, Gina M.: The Performance of Respirator Cartridges at High Relative Humidities, Masters Essay, Graduate School of Public Health, University of Pittsburgh (1986).

12. Perry, R. H., and Green, D.: Perry's Chemical Engineers' Handbook, Sixth Ed., Section 16, Page 17, McGraw-Hill (1984).

13. Javorsky, M.: The Performance of a Prototype Respirator Cartridge at High Relative Humidity, Masters Essay, Graduate School of Public Health, University of Pittsburgh (1986).

14. Dubinin, Mo, Erashko, I. T., Kad 1 ec, 0., Ulin, V. I., Voloshchuk, and Zolotarev, P.: Rinetics of Physical Adsorption by Carbonaceous Adsorbents of Biporous Structure, Carbon 13:193-200 (1975).

15. Dubinin, Mo: New Experimental and Theoretical Investigations into Kinetics of Physical Adsorption by Microporous Adsorbents, Pure App 1. Chem. 48:407-14 (1976). 
16. Bering, B. P., and Serpinsky, V. V.: Thermodynamic Tests for the Applicability of the Potential Theory of Adsorption, Dok 1. Akad. nuak, S.S.S.R. $153: 129$ (1963).

17. Bering, B. P., Serpinsky, V. V., and Surinova, S. I.: Joint Adsorption of Binary Mixtures of Vapors and Activated Carbon, Izv. Akad. nauk. S.S.S.R. 1965:769-76.

18. Swearengen, P. M., and Weaver, S. C.: The Effects of Organic Vapor Mixtures on Respirator Service Life - II, Paper 324, The American Industrial Hygiene Convention, Dallas, TX (1986).

19. Moyer, E. S.: Methyl Isocyanate Studies, Part 1: Air-Purifying Respirator Cartridge Evaluations, Paper 325, The American Industrial Hygiene Convention, Dallas, TX (1986).

20. Yoon, Y. H.: . The Effect of Humidity on Respiratory Cartridge Service Life, Paper 326, The American Industrial Hygiene Convention, Dallas, TX (1986).

21. Wood, G.O.: Effects of Humidity on Air-Purifying Beds, Paper 328, The American Industrial Hygiene Convention, Dallas, TX (1986).

22. King, W. P., Roberts, C. C., and Mckee, E. S.: Effects of Environmental Conditions on the Removal of Methyl Iodide by Air Purifying Respirators, Paper 329, The American Industrial Hygiene Convention, Dallas, TX (1986).

23. Ackley, M. W.: Chemical Cartridge Respirator Performance: 1,3 Butadiene, Paper 331 , The American Industrial Hygiene Convention, Dallas, TX (1986). 
PRACTICAL TEST REQUIREMENTS FOR GASEOUS CONTAMINANT REMOVAL EQUIPMENT

Richard D. Rivers, Vice President

Environmental Quality Sciences

P.0. Box 2147, Louisville, RY 40204

Intel 1 igent design of gaseous contaminant removal equipment for general ventilation systems is surely one of the more difficult aspects of building technology. The designer is merely guessing if he does not have data for the following factors for the space in question.

Chemical and physical properties of gaseous contaminants

Contaminant concentrations or internal generation rates

Allowable contaminant concentrations within the protected space

Air flow requirements and level of mixing

In addition, the designer must know the following things about the contaminant-removal equipment:

Removal efficiency at operating levels

Life or maintenance requirements at operating levels

Sensitivity to humidity, temperature and contaminants

Nature of effluent products, if any, created by the equipment

Energy Consumption

Capital and maintenance costs

The difficulty of the problem becomes apparent with the first two listed factors. In most cases, the gaseous contaminants present are not entirely identifiable, nor are their concentrations known, even as averages. Information on the contaminant levels which can be tolerated is scarce, and argued over by experts. Yet the designer must seek to control these unknowns to innocuous levels, using manufacturers' data for the remaining factors in the table. Much of this 'data' is in the form of 'excellentgood-fair-poor. Where numerical information is available, it reports the results of tests by the methods listed in Table 1.

The first listed test method, the complete isotherm, does allow approximate calculation of field performance for a set of assumed contaminants and operating conditions. The translation of the isotherm into filter penetration and 1 ife is, however, a major undertaking in chemical engineering, and far beyond the interest of HVAC system designers. Test methods 2 through 5 provide only order-of-magnitude performance data, and are essentially quality-control procedures. Test method 6 offers the best hope for the HVAC system designer. It provides data on at least one contaminant, with the major effects of mass transfer and sorbtion evaluated 
at the operating conditions of the test. If these conditions are met by the installed system, there is reason to believe it will deliver performance equivalent to that obtained in the test. This discussion seeks to determine parameters for a useful and practical gaseous-contaminant penetration-vs-load test.

1. A1 1 owable concentrations. The concentrations allowable for a given contaminant can be dictated by considerations of safety, health, annoyance, or material damage. Table 2 ists a few contaminants, with levels for human safety (IDLH, the level immediately dangerous to 1 ife and health); health (TWA8, 8-hour time-weighted averages acceptable for 40-hour work weeks); and annoyance (OTH, odor threshold for $50 \%$ of the population). No values are 1 isted for material damage, since these depend on the materials present. It is possible, however, for material damage to be the critical factors. The values 1 isted for IDLH and TWA8 are taken from OSHA and ACGIH tables $[10,11]$, with the exception of the values for sarin, a chemicalwarfare nerve agent. The contaminants were selected to illustrate the wide range of values for any one allowable concentration index - and also that no one index governs the allowable level. In most cases odor threshold is the lowest level, and this determines the maximum concentration al lowable for the chosen contaminant. In the case of $\mathrm{CO}$ and $\mathrm{CO}_{2}$, which are odorless (infinite OTH), odor has no influence, and TWA 8 sets the allowable concentration.

Much adsorption data are available for gas-mask canisters. Such canisters reduce a relatively high concentration (often IDLH) outside the mask, upstream of the filter, to an acceptable TWA8 level inside the mask, downstream of the filter. In a general-ventilation situation, however, the air from the conditioned space appears upstream of the filter. The concentration entering the filter will be at far lower levels than in the gas-mask case. A test for general-ventilation filters needs to reflect this difference.

TABLE 1. EXISTING TEST METHODS FOR GASEOUS CONTAMINANT ADSORBERS

Test

1. Complete Isotherm

2. One-Point Isotherm

3. Breakthrough

4. Integrated Penetration

5. Odor Breakthrough

6. Penetration-vs-Load

* $S$ = static equilibrium (no air flow)

$\mathrm{D}=$ dynamic (air flow through test bed)

** Volume of adsorbate in bed = function of (adsorbate concentration in gas)
Description/Example

See $* *$ below; [1]

ASTM D3467 ( CC14) [2]

Time to set penetration; BuMines ' $23 B$ ' [3], MIL STDs. $[4],[5]$

Penetration at set load; ASTM D3803 [6]

Perceived Breakthrough [7]

Nelson [8], Ostajic [9] 
TABLE 2. SELECTED GASEOUS AIR POLLUTANTS:

TOXICITY, ODOR AND BOILING POINT

Compound

Acrolein

Ammonia

Carbon dioxide

Carbon monoxide

Carbon disulfide

Carbon tetrachloride

Formaldehyde

Hydrogen sulfide

Ozone

Sarin (GB)

IDLH
13
350
90000
1650
1500
1800
124
420
20
$0.40 ?$

Concentration, $\mathrm{mg} / \mathrm{m} 3$

TWA8

0.25

35

9000

55

60

60

4

30

2

0.000003 ?
BP

$\operatorname{deg} \mathrm{C}$

52

$-33$

$-78$

$-192$

46

77

97

$-60$

$-112$

158

IDLH = Immediately dangerous to life and health

TWA8 = 8-hour time weighted average, allowed for 40-hour work week

OTH = Odor threshold

BP = Boiling point at $760 \mathrm{mmHg}$

2. Efficiency (or Penetration ${ }^{1}$ ) and Sorber Life. These two filter parameters are inextricably related. The instantaneous value of sorber penetration is dependent on many factors, including:

a. Whether the filter material is a physical adsorber, an adsorber, chemisorber, or a catalyst.

b. The surface properties of the sorption material.

c. Sorber granule size and packing density.

d. The physical and chemical properties of the contaminant.

e. The temperature and humidity of the carrier gas.

f. Carrier gas bed velocity.

g. Bed depth.

h. Contaminant concentration.

i. The previous history of the sorption material, especially its sorbed load of contaminant and water vapor.

$j$. Competition from other contaminants.

k. Generation of contaminants by the sorption device.

This large list of factors means that test conditions need to be fully specified if the results are to be interpreted intelligently. Factors a through $d$ are fixed in evaluating a given adsorber/adsorbate combination. If we are only interested in one set of operating conditions, factors e through $\underline{h}$ can be at the values of that set; if we seek to determine the effect of variations in the factors, testing will have to be done at several values of each, unless the function relating each factor to penetration and life is known.

(1) When efficiency is near $100 \%$, as is often the case for fresh sorption devices, it is better to use the term penetration ( $=100$ - efficiency, in percent), the measure of what passes the filter, rather than what is caught. 
A common view of adsorbtion devices is that they have $0 \%$ penetration until a sudden 'breakthrough' to $100 \%$ penetration. This is not true. Actual performance is as in Figure 1 , which shows penetration for a physical adsorber or chemisorber as a function of the amount of captured adsorbate, for two different inlet concentrations. When the adsorbent bed is new, penetration is low, but not zero; it rises slowly as adsorption sites are filled, then very rapidly when almost all sites are filled. The absolute level of downstream concentration will be lower when the inlet concentration is low, but the percent penetration will generally be higher at low in let concentrations. Note that the two load scales are different, and that breakthrough occurs at a lower adsorbate load for the low inlet concentrations than for high inlet concentrations. Replenished adsorbers, such as spray towers, maintain a steady penetration level, and true catalysts show a slowly increasing penetration as they become 'poisoned'. Both of these, however, have some penetration, and its value is a function of the inlet contaminant concentration among other things.

Total load for $1000 \mathrm{mg} / \mathrm{m} 3$ inlet concentration

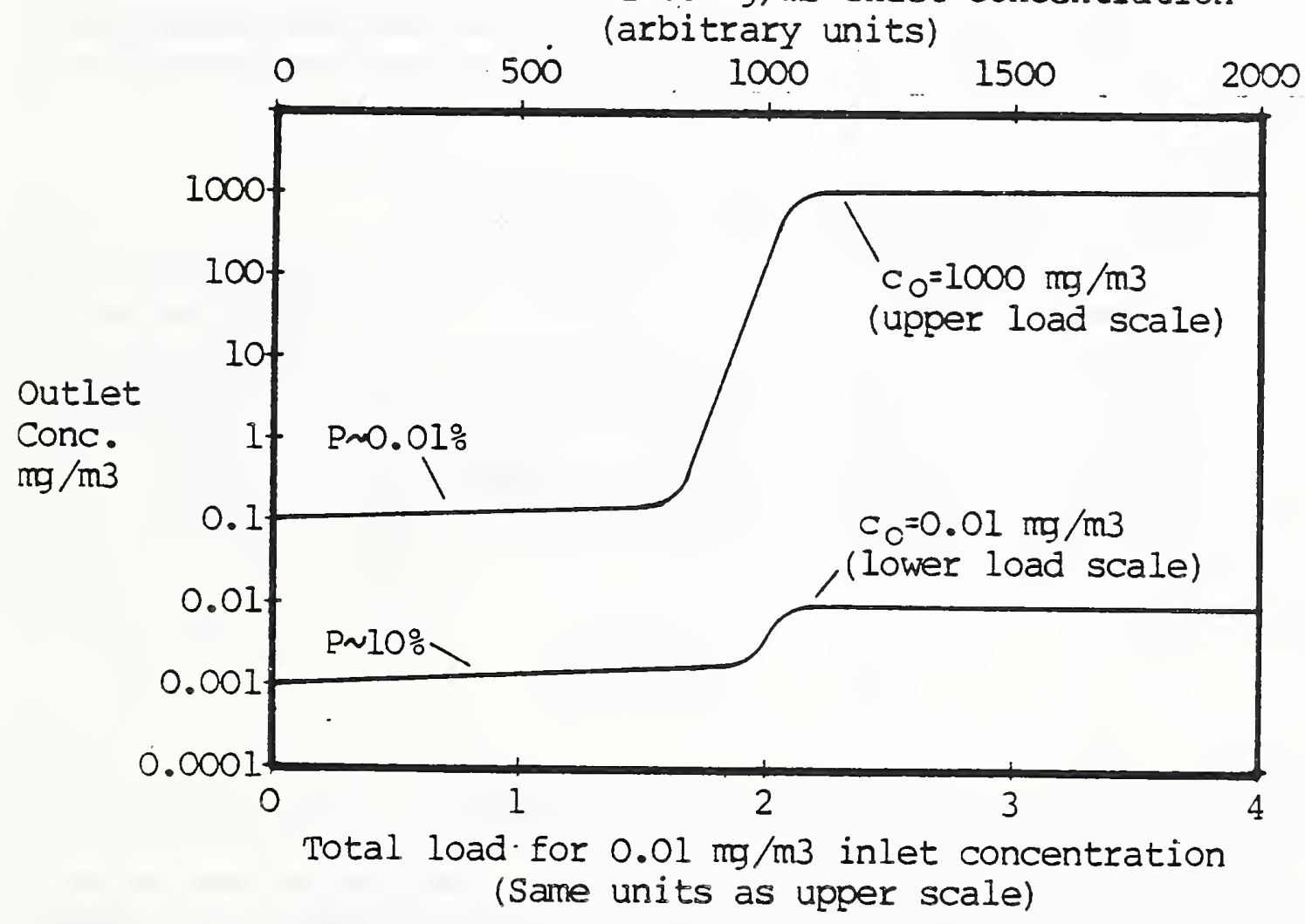

Figure 1. Penetration-vs-Load for Typical Adsorber

Evaluating every adsorber for ranges of all the 1 isted factors is impractical. It is important to narrow down the number and ranges of parameters as much as possible. To do this, a simplified mass-balance model of the contaminant-control system (Figure 2) will be helpful. The model has an internal contaminant source (G), make-up air (ventilation air, V) from outdoors, recirculation(R), and a filter with penetration P. We neglect infiltration and exfiltration, and removal mechanisms like adsorption on which we would like to reduce to zero; it is scarcely proper to take advantage of them in designing a system. 


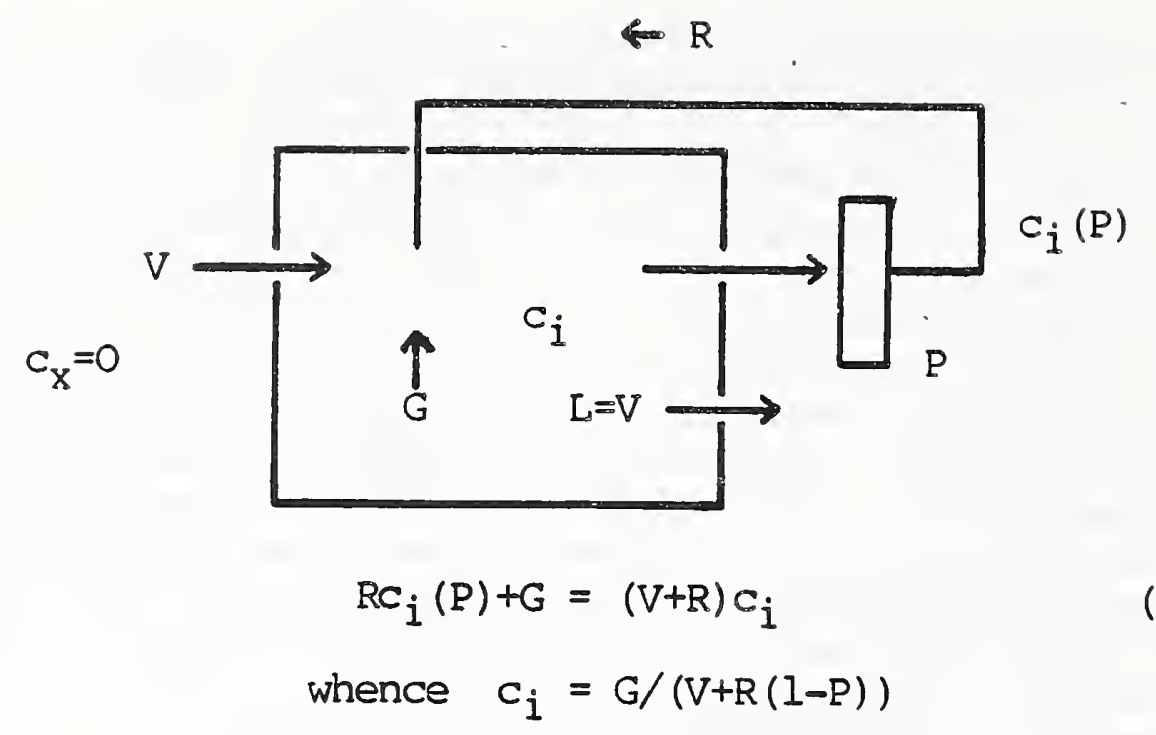

Figure 2. Mass-Balance Filtration Model

Table 3 lists some factors which allow us to estimate the concentrations met in typical protected spaces, hence which might make reasonable test concentrations.

TABLE 3. INLET CONCENTRATION CALCULATIONS

Indoor Contaminant Generation Factors

\begin{tabular}{lrrrr} 
Space Type: & Arena & Office & \multicolumn{1}{c}{ Units } & Reference \\
Population: & 1.5 & 0.2 & occupants/m2 & {$[12]$} \\
Area: & 7500 & 100 & $\mathrm{~m} 2$ & {$[12]$} \\
Smoking: & 2.0 & 0.7 & cigarettes/hr/occ & {$[14]$} \\
Ventilation: & 8.5 & 25.5 & $\mathrm{~m} / \mathrm{hr} /$ occ & {$[12]$} \\
Human Emissions & & & & {$[13]$} \\
Hydrogen sulfide: & 0.02 & 0.015 & $\mathrm{mg} / \mathrm{hr} /$ occ & {$[13]$} \\
Ammonia: & 35 & 20 & $\mathrm{mg} / \mathrm{hr} /$ occ & \\
Smoking & & & & \\
Volatile organics: & 15 & 15 & $\mathrm{mg} / \mathrm{cigarette}$ & {$[14]$}
\end{tabular}

These factors applied to the mass-balance model give concentrations which are 1 isted in Table 4. The minimum steady-state concentration exists when the filter has zero penetration, the maximum when it has $100 \%$ penetration. There are some striking things about the results. The steady-state concentrations due to internal sources are below or near the odor thresholds; they are far below the levels at which the usual tests are run. This may explain a lot of testimonials from users to the effectiveness of odor-control devices when they in fact have near-zero performance. The systems simply have enough infiltration or make-up air to push the average contaminant concentrations below odor thresholds. With the space, there may be smoking or other local odor sources which are easily detectable; yet the return air from the zero-efficiency filter system will sme 11 'fresh' hence the filter is perceived to be doing a fine job. From the standpoint of toxicity or material damage, of course, a zero-efficiency devices does nothing. 
TABLE 4. INLET CONCENTRATION CALCULATIONS

Contaminant Concentrations and Loads

Calculated from Factors in Table 3a Using Eq. 2

\begin{tabular}{|c|c|c|c|}
\hline Space Type: & Arena & Office & Units \\
\hline Total Ventilation: & 95600 & 510 & $\mathrm{~m} 3 / \mathrm{hr}$ \\
\hline Recirculation: & 1816400 & 2040 & $\mathrm{~m} 3 / \mathrm{hr}$ \\
\hline$\%$ Recirculation: & 95 & 90 & $\%$ \\
\hline \multicolumn{4}{|l|}{ Human Emissions } \\
\hline Hydrogen sulfide: & 225 & 0.3 & $\mathrm{mg} / \mathrm{hr}$ \\
\hline Min, ma conc: & $10^{-4}, 0.002$ & $10^{-4}, 0.0006$ & $\mathrm{mg} / \mathrm{m} 3$ \\
\hline Odor threshold: & 0.007 & 0.007 & $\mathrm{mg} / \mathrm{m} 3$ \\
\hline Ammonia: & 394000 & 400 & $\mathrm{mg} / \mathrm{hr}$ \\
\hline Min, $\max$ conc: & $0.2,4.12$ & $0.16,0.78$ & $\mathrm{mg} / \mathrm{m} 3$ \\
\hline Odor threshold: & 35 & 35 & $\mathrm{mg} / \mathrm{m} 3$ \\
\hline \multicolumn{4}{|l|}{ Smoking Emissions } \\
\hline Volatile organics: & 169000 & 300 & $\mathrm{mg} / \mathrm{hr}$ \\
\hline Min, $\max$ conc: & $0.09,1.77$ & $0.12,0.59$ & $\mathrm{mg} / \mathrm{m} 3$ \\
\hline Odor threshold: & $?$ & $?$ & $\mathrm{mg} / \mathrm{m} 3$ \\
\hline
\end{tabular}

This type of calculation shows that a useful test procedure for gaseous contaminant control devices must somehow evaluate the devices at odorthreshold levels. This is not easy. Both feeding and detection at low levels are difficult, and loading a filter at low concentration is timeconsuming. The time involved with available sorption materials may not be as long as one would expect from equilibrium capacity (activity) data, however. Nelson et al [8] ran data on the adsorption capacity of gas-mask carbon canisters fro several solvent vapors in the range 100-1000 ppm. They found that filter 1 ife (time to $10 \%$ penetration) was related to concentration by eq. 3 :

$$
t=k c^{b}
$$

where $t$ is breakthrough time, $C$ concentration, and $k$ and $b$ constants. For one tested compound, hexane, $b$ was -1 , which one would expect if there were no concentration effect. In general, however, $b$ was about -0.67 . If this value is generally true, then the life of a carbon adsorber at 1 ppm in let concentration would be about 102 times the 1 ife measured at 1000 ppm. Measured breakthrough times at 1000 ppms were typically 2 hours, hence one would expect 1 ives of 200 hours at $1 \mathrm{ppm}$. This seems to agree with other reported low-concentration data; Hanna and Ruehner [15], for examp le, show 1 ives of 150 hours for activated carbon and Purafil (activated alumina impregnated with potassium permanganate) on acrolein at 0.25 and 0.5 ppm respectively, with stay-times of $0.075 \mathrm{~s}$. Jonas and Svirbely [16] showed a 1 ife at $10 \%$ penetration of 17.5 minutes, using 14.4 ppm of carbon tetrachloride, $0.5 \mathrm{~s}$ stay-time, and Pittsburgh BPL carbon. While lowconcentration testing may be tedious for adsorbate-sorber combinations which work well, in many cases the test will be completed very quickly.

Ostajic [9] reports on attempts to speed up testing by loading at high concentration and switching occasionally to a low concentration for 
penetration tests. This is not feasible in general, for molecules that would eventually migrate to secure capture sites if they were deposited slowly may be very poorly retained if deposited rapidly. When the feed at high concentration was switched to low concentration, the loosely bound adsorbate molecules eluted out of the bed, actually at higher concentration than the then existing feed. Ostajic suggested that automated test equipment would make low-concentration test possible over periods of several hundred hours. This seems reasonable, since we are not seeking quality-control data, but data to characterize a specific sorber formulation for design purposes.

Life data is obtained simultaneously with penetration data. It is, however, necessary to define some breakthrough penetration which defines the "Iife" of the sorber, if that is to be published. Nelson's $10 \%$ of upstream concentration is probably satisfactory for general ventilation purposes, since upstream concentration has already been set at an acceptable level for the space. It is unlikely that a sorber with greater than $10 \%$ penetration will be economically competitive with simple ventilation. It is a reasonable assumption that the make-up air will contain significant amounts of the EPA 'Criteria Pollutants' - sulfur dioside, carbon monoxide, nitrogen dioxide, ozone, and nonmethane hydrocarbons. In addition, methane is preseat at about the $1 \mathrm{mg} / \mathrm{m} 3 \mathrm{level}$, and water vapor. Designers need to know the influence of these contaminants on the penetration and life of the sorbtion devices they evaluate. They should therefore be present in the air flow used to test sorbers. If the concentration of test contaminants is multiplied by some factor to speed up the test, then these criteria pollutants should be present in their usual concentration multiplied by that factor. We might take the usual concentrations to be the EPA primary air-quality standards [17], as listed in Table 5, exerpted here.

\section{TABLE 5. EPA PRIMARY STANDARD FOR CRITERIA AIR POLLUTANTS}

Pollutant

Sulfur Dioxide

Nitrogen Dioxide

Ozone

Carbon Monoxide

Nonmethane Hydrocarbons

$$
\text { Primary Standard, } \mathrm{mg} / \mathrm{m} 3
$$

0.08

0.10

0.24

10.0

0.16

Since filters will generally be located in the recirculation loop, design humidity conditions will generally be the indoor design condition, perhaps $50 \% \mathrm{RH}$ at $23 \mathrm{C}(73 \mathrm{~F})$. Chemisorbers cannot operate at bone-dry conditions, while physical adsorbers suffer from high humidities. If the loading is carried out at actual allowable concentration levels, there is no problem; use $50 \% \mathrm{RH}$ at $23 \mathrm{C}$. If the contaminant concentrations are multiplied by a factor as large as 2, however, the most prevalent contaminant of al1, water vapor, will be at saturation. There seems to be little choice but to run the test at the $50 \% \mathrm{RH}, 23 \mathrm{C}$ condition. The air entering the test rig should be converted to 'zero air' by filtering through particulate filters followed by deep beds of sorption media able to remove acid gases as well as ambient organics and ozone to well below test conditions. Controlled amounts of test contaminants, including water vapor, would then be added to the test air flow. 
3. Nature of Effluents. The test procedure must verify that any reaction products from the filter are innocuous (including being below odor thresholds). This is rarely a problem with physical adsorbers, unless some catalytic effects are present. However, physical adsorbers may give up adsorbate when unpolluted air passes through the bed; hence a period of elution using unpolluted air at test temperature and humidity must be a part of the test.

4. Air Flow. This will normally be determined by factors not related to contaminant filtration, such as thermal control and satisfactory air distribution. Obviously, test conditions should match manufacturer design flow range for the device or material tested. The test canister described in ASTM D3803 (Figure 3) is of practical size for smal1-scale tests of granular adsorbers, chemisorbers and catalysts. This canister, which is intended for a face velocity of about $0.2 \mathrm{~m} / \mathrm{s}(40 \mathrm{ft} / \mathrm{min})$, is small enough to allow convenient control of all test conditions, yet large enough to average out filter media variations and avoid significant edge effects. Scrubbers and other devices may require larger test prototypes, but test costs encourage use of the smallest representative test prototypes.

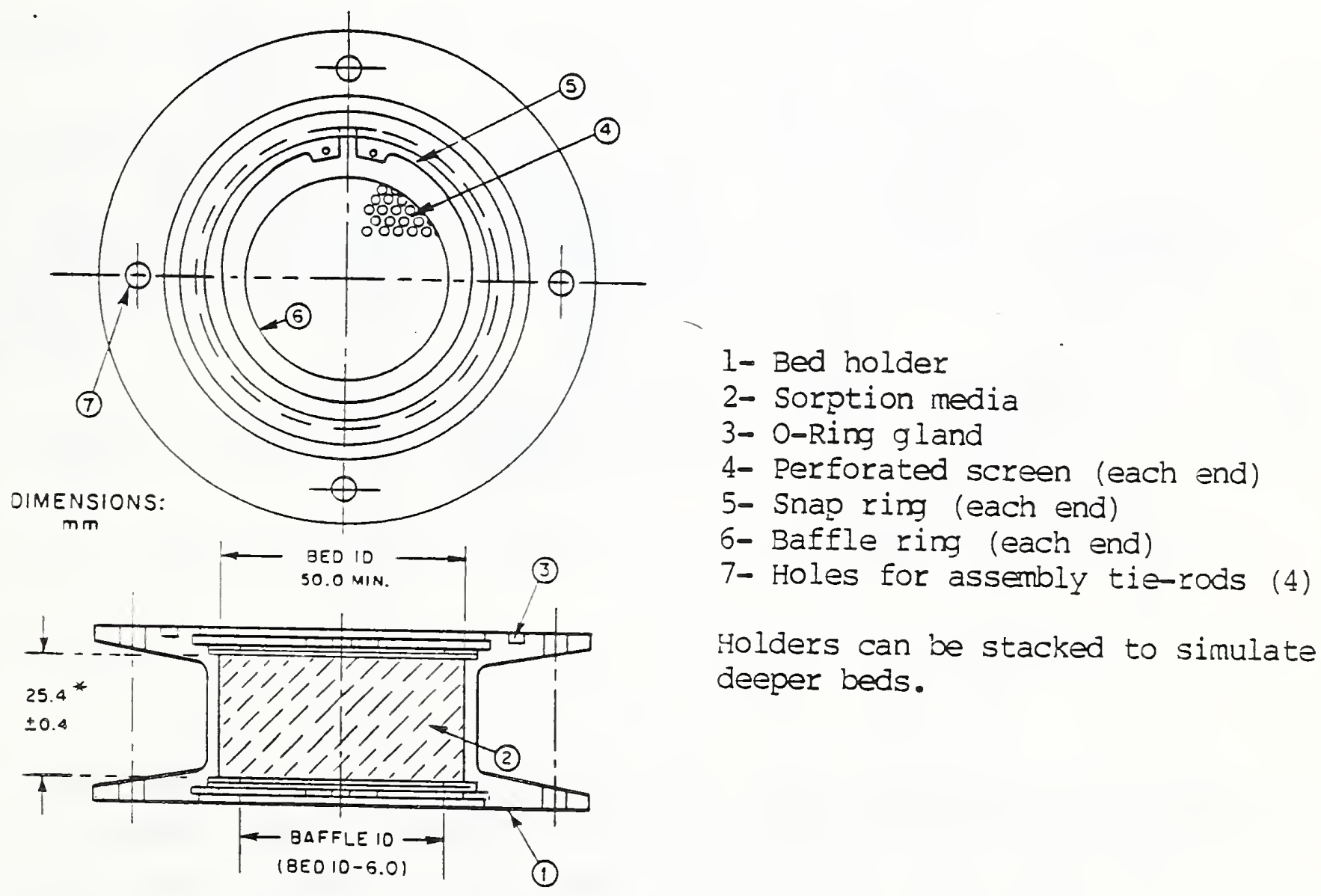

Figure 3. ASTM D3803 Test Canister

5. Energy Consumption. For granular beds, energy consumption is a matter of bed pressure drop. In a practical filter device this is not the drop through the granular bed alone, but includes the drop through the retaining screens, and the inlet and outlet passages to the beds. Pressure drop must be measured on ful1-size, typical unit configurations, and be presented as a function of gas flow or face velocity. Wet scrubbers require energy for pumping spray water, and there may be other energy inputs. 
6. Capital and Operating Costs. These must be fully identified so that system designers can do meaningful 1 ife-cycle costing. This means that reasonable estimates must be made of things like waste of media and manhours for reloading cells. If maintenance costs are based on reactivation of the sorber material, then performance data must be given on reactivated material.

7. Design of Test Facility. The design of the test facility is not simple. ASTM D3803 gives a schemaric (see Figure 4) for a system intended to test activated carbon at botb room humidity conditions and near saturation. This may be a little ornate for testing adsorbers for general ventilation, and indeed, many of the problems identified in ASTM D3803 originate in its $95 \% \mathrm{RH}$ test. Nevertheless, the schematic shows the essential piping, thermal controls, contaminant feed systems, and measurement of flow, temperature and pressure. Any facility proposed for industry-wide use should be adaptable to a wide range of contaminants, including those that condense out at room temperature. There is a large body of literature on generating and detecting specific contaminants.

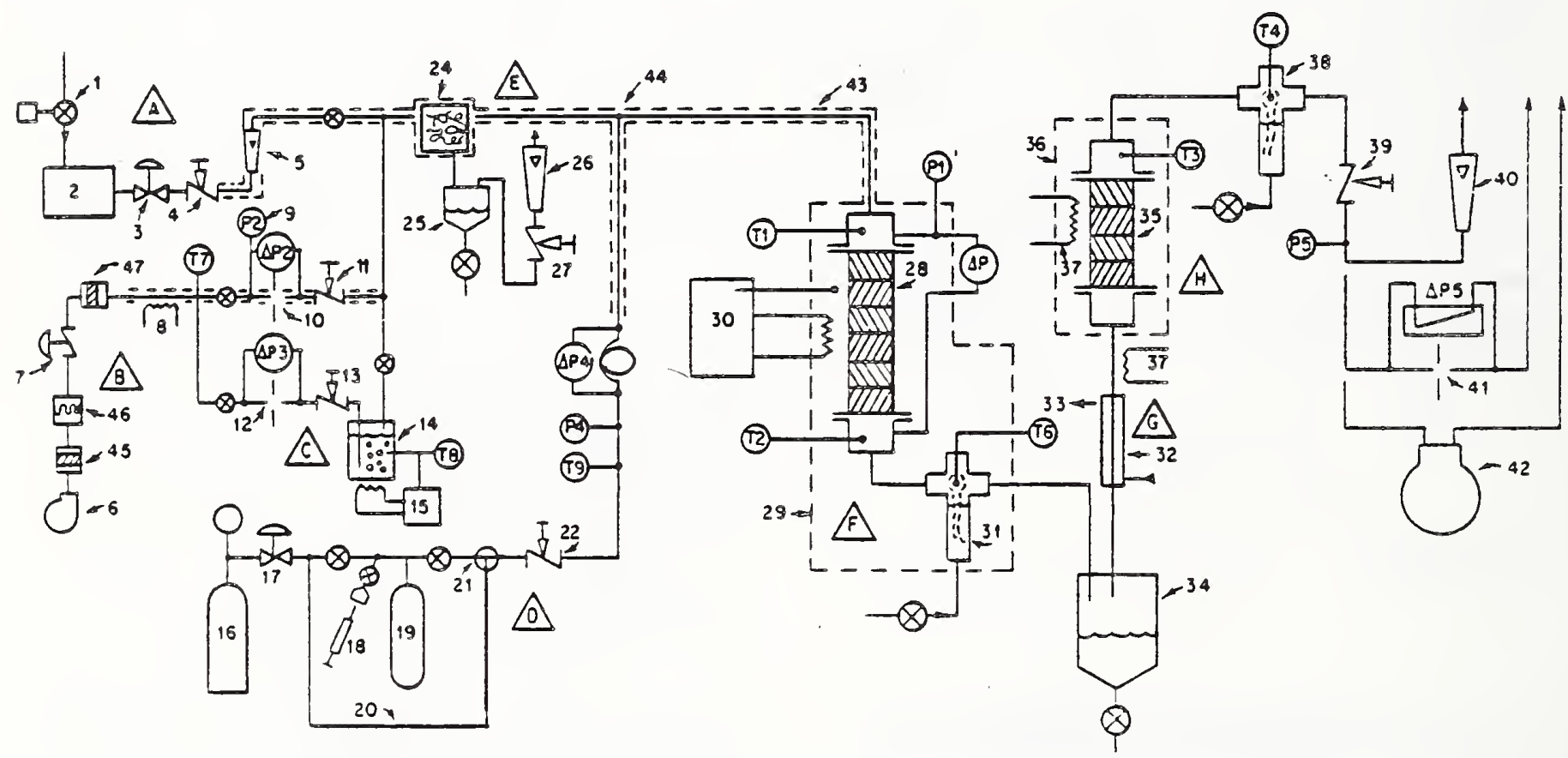

Figure 4. ASTM D3803 Gaseous Contaminant Test System Schematic

8. Suggested Contaminant List. A standard test for general-ventilation service might include contaminants from major chemical groups, (Table 6 ).

Feeding and detecting such a 1 ist simultaneously would pose severe problems, and would not simulate any real situation. The contaminants would therefore be fed in separate tests, along with the criteria air contaminants at the concentrations 1 isted in Table 5. "Nonmethane hydrocarbons" could be simulated by 2,2,4-Trimethylpentane, and methane itse $1 \mathrm{f}$ would be added at $1 \mathrm{mg} / \mathrm{m} 3$. Where a test is run to evaluate penetration and life on sulfur dioxide, ozone, or 2,2,4-Trimethylpentane, these would be left out of the 'background' feed. Even this pattern would require up to seven simultaneous feeds of contaminant gases, though some would be at rather low levels. It might be possible to determine which of these have little or no influence on the aging of different categories of 
sorbers, and to eliminate them from the required feed if they have no effect. The sorber performance on these contaminants would have to be measured and reported if this alternate feed was chosen.

TABLE 6. SUGGESTED TEST CONTAMINANTS

$\begin{array}{llrrr}\text { Contaminant } & \text { Group } & \begin{array}{c}\text { Suggested } \\ \text { mg/m3 }\end{array} & \text { MW } & \begin{array}{r}\text { BP } \\ { }^{\circ} \mathrm{C}\end{array} \\ \text { Sulfur dioxide } & \text { Acid Gas } & 1.2 & 64 & -10 \\ \text { Formaldehyde } & \text { Aldehyde } & 1.2 & 30 & 97 \\ \text { Methanol } & \text { Alcohol } & 260 & 32 & 65 \\ 2,2,4-\text { Trimethylpentane* } & \text { Alkane } & 1450 & 114 & 98 \\ \text { 2-Butanone (MER) } & \text { Retone } & 30 & 72 & 80 \\ \text { Ozone } & \text { Oxidant } & 0.2 & 48 & -112 \\ \text { 1,1,1 Trichloroethane } & \text { Halocarbon } & 1.1 & 133 & 75\end{array}$

* = iso-octane

where special capabilities exist in the sorber device, such as the ability to remove carbon monoxide, that contaminant would be added to the 1 ist.

Some saving of test time is perhaps possible by simultaneous measurement of penetrations at several bed depths. A sampling valve could be switched from one sequential bed sampling point to the next, using the same detector. Both experimental and theoretical work is needed to validate a test procedure and provide ways in which designers can estimate performance at conditions different from the standard test, and on other contaminants.

REFERENCES

[1] Many texts, for examp le Chemisorption, D. O. Hayward and B. M. W. Trapne11, Butterworths, Washington, 1964.

[2] ASTM D3467, Carbon Tetrachloride Activity of Activated Carbon, Annual Book of ASTM Standards, Part 30. ASTM, Philadelphia, PA 19103.

[3] Department of Interior, Bureau of Mines: Respiratory Protective Devices: Tests for Permissibility. 30 CFR 11 , subparts $I$ and L.

[4] MI1-C-13724: Charcoa1, Activated, Impregnated, ASC. Nava 1 Publications and Forms Center, Philadelphia, PA 19120.

[5] MIL-STD-282: Filter Units, Protective Clothing, Gas-Mask Components and Related Products: Performance Test Methods (1956) (address: see $\operatorname{Ref} .6$ ).

[6] ASTM D3803, Radioiodine Testing of Nuc lear-Grade Gas-Phase Adsorbents, Annual 1 Book of ASTM Standards, Part 30. ASTM, Philadelphia, PA 19103 .

[7] ASTM E544, Referencing Suprathreshold Odor Intensity. Annual Book of ASTM Standards, Part . ASTM, Philadelphia, PA 19103. 
[8] G. 0. Nelson and A. N. Correia, Respirator cartridge efficiency studies: VIII, Summary and Conclusions. Am. Indus. Hyg. Assn. J. $37: 514-525(1976)$.

[9] N. Ostajic, Test method for gaseous contaminant removal devices. ASHRAE Transactions, 1985, Vo1. 91, Pt. 2.

[10] Code of Federal Regulations Title 29 (Labor) $1900.1000 \mathrm{ff}$ (annual).

[11] Threshold Limit Values for Chemical Substances in the Work Environment. ACGIH, Cincinnati, OH 45211 (annual).

[12] ASHRAE Standard 62-1981, Ventilation for Acceptable Indoor Air Quality. ASHRAE, Atlanta, GA 30329 (1981).

[13] Bioastronautics Data Book (p. Webb,ed) NASA SP-3006 (1964) (NTIS).

[14] D. M. Burns, Involuntary Smoking. In Smoking and Health: A Report of the Surgeon General DHEW PHS 99-50066 (1979).

[15] G. F. Hanna and R。 I. Ruehner, Critical factors in odorant measurement and control. ASHRAE Symposium "Odors and Odorants: The Engineering View", ASHRAE, Atlanta, GA 30329 (1969).

[16] L. A. Jones and W. J. Svirbely, The kinetics of adsorption of carbon tetrachloride and chloroform from air mixtures by activated carbon.

[17] ASHRAE Handbook - 1985 Fundamenta 1s, Ch. 11.6., ASHRAE, At 1anta, GA 30329 . 
Cyrus M. Bosworth

Cyrus M. Bosworth and Assoc.

Waldoboro, Maine

\section{INTRODUCTION}

The ASHRAE Technical Committee 2.3 (and 5.4) have been looking for a standardized test procedure for more than 25 years. ASHRAE has standardized procedures for particulate removal filters but not for gas removal. So, the suppliers of gas removal equipment have developed their own procedures (using procedures published by Dr. Amos Turk and others). I would like to relate some of these which I used when I was a "supplier" and experiences when I was a "user" of gas removal equipment.

\section{BÄSES FOR COMPARISON:}

There are three important parameters to be evaluated:

1. How much is left in the air,

2. How long will it last and do its job, and

3. How much power is required?

Other parameters can be important, such as the thickness required, crosssectional area, maximum velocity, and especially the price.

\section{THEORETICAL EVALUATIONS:}

The designer of the gas removal equipment may need to evaluate the theoretical surface required. He may calculate the mass transfer coefficient needed, if he knows (a) what the gas is, (b) the concentration of the gas in the air, and (c) how much it must be reduced; see equation 1.

$$
\mathrm{N}_{1}=\mathrm{k}_{\mathrm{g}} \mathrm{A}_{t}\left(\mathrm{P}_{1}-\mathrm{P}_{\mathrm{e}}\right)
$$

where: $N_{1}=$ Quantity removed

$$
\begin{aligned}
& \mathrm{k}_{g}=\text { Mass transfer coefficient } \\
& A_{t}=\text { Transfer area } \\
& \mathrm{P}_{1}=\text { Partial pressure of gas in air } \\
& \mathrm{P}_{e}=\text { Partial pressure of gas on adsorbent }
\end{aligned}
$$

The designer would like to know the porosity of dry adsorbent, what packing density, in order to have low power loss. Any of a number of equations can be used to correlate pressure drop with velocity and the momentum loss through the specific media. This is also true for the developer of liquid adsorption systems, in pressure loss through the media and prevention of 1 iquid carry-over.

The ratio of the quantity of gas removed divided by the power required to move the air through the media, has been used to evaluate both the dry adsorbent systems and the wet air scrubber systems, and to make comparisons. I developed a ratio I called the MERIT FACTOR and presented it as part of a paper over 20 years ago. We used it to compare gas removal 
equipment, but I never heard of anybody else using this " $B$ " number (see equation 2).

$$
B=\frac{k_{g} A_{t}}{P f^{D}}
$$

where: $B=$ Merit factor

$$
\begin{aligned}
\mathrm{R}_{\mathrm{g}} & =\text { Mass transfer coefficient } \\
\mathrm{A}_{\mathrm{t}} & =\text { Surface area (transfer) } \\
\mathrm{D}^{\mathrm{p}} & =\text { Pressure drop } \\
& =\text { Air flow rate }
\end{aligned}
$$

PRACTICAL EVALUATIONS:

Figure 1 shows a typical air conditioning equipment sequence. The return air is drawn into the system along with the outside ais and mixed in the mixing section. The outside air may be prefiltered and pre-heated, and is controlled by the dampers at the inlet. The filter section usually contains only a roughing filter to protect the coils from dirt. Shown here is a better grade particulate filter to protect the gas removal filter. Next is the coil section with cooling coil first and the re-heat coil second. Finally the fan in the fan section pulls the air through the equipment and fills the ducts to distribute the conditioned air to the space. The cooling coil on humid summer days removes water vapor from the air and at the same time removes many gaseous contaminants. However, on the off cycle, the coil warms, evaporates the water and puts the gaseous pollutants back into the air, sometimes causing complaints from the occupants.

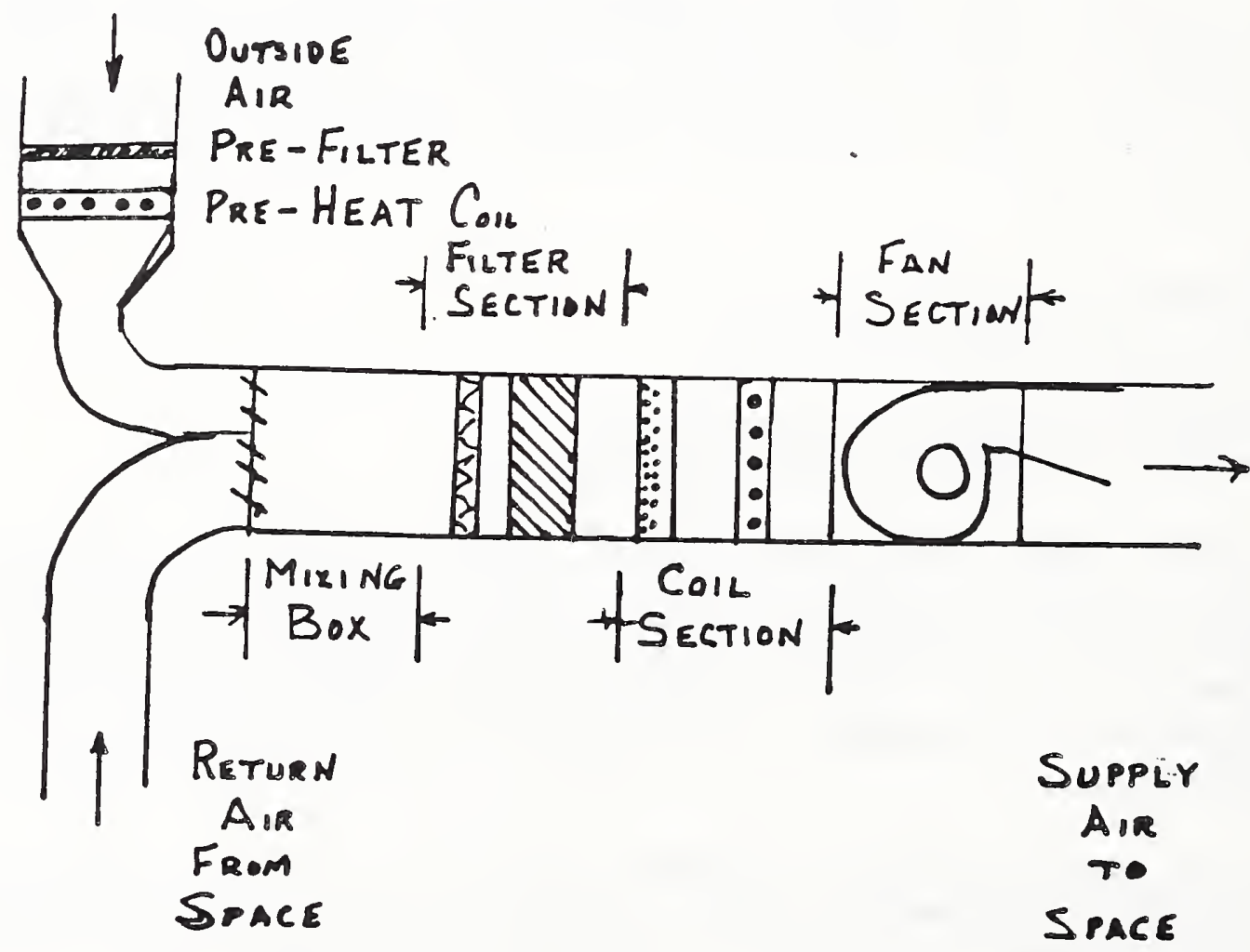

Figure 1 
A. Figure 2 shows a "once through" test. There are no end of variations, but the essentials are a source of fairly clean air, a means to add a pollutant gas, obtaining a sample upstream that is we 11 -mixed, a test section for the gas removal device in which pressure drop can be measured and a we 11-mixed downstream sample can be taken, and finally the fan or other means for moving the air. Reference 4 present one procedure.

\section{Once - Thru}

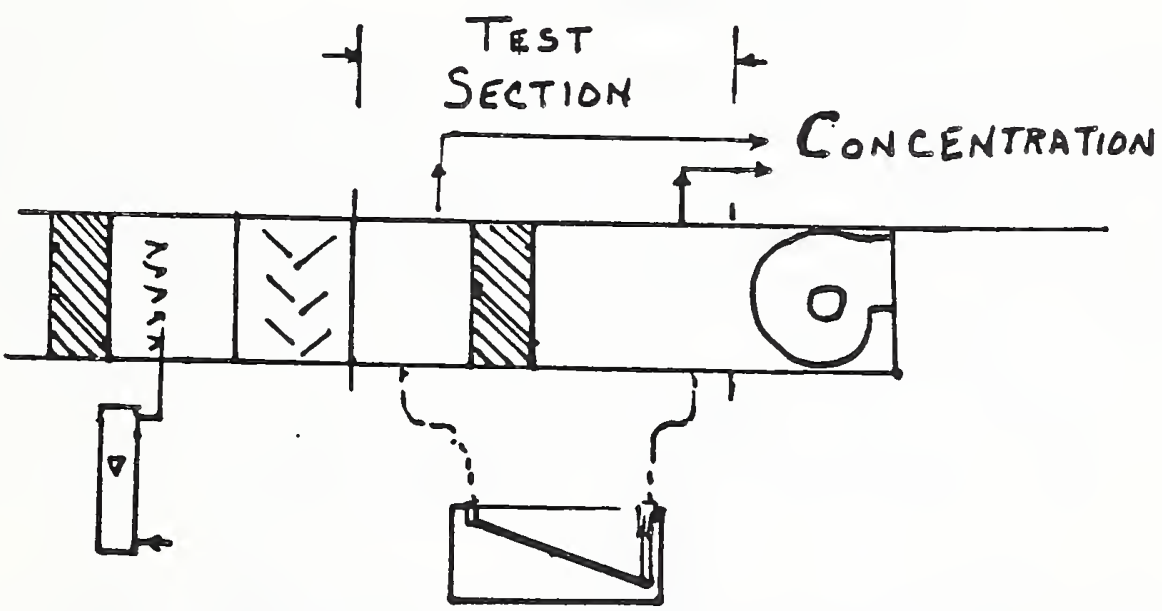

Figure 2

The results could be reported as concentration vs time, but we usually measure pollutant removal per pass and pressure drop at several velocities, some higher and lower than whose we expect to use the device. We usually express the removal as efficiency per pass, or Eff. $=100(\mathrm{Cu}-\mathrm{Cd}) / \mathrm{Cu}$.

We used this procedure in situ in the chemical plant for evaluating and comparing air scrubbers in the exhausts, (Figure 3) also a variation of the test when we evaluated air washers used to air condition textile plants for removing formaldehyde in the air. Figure 4 shows a high velocity air washer which has sprays of chilled water that removes particulates as well as pollutant gases.

\section{ELEGTROLYTICALLY REGENERABLE SCRUBBER}

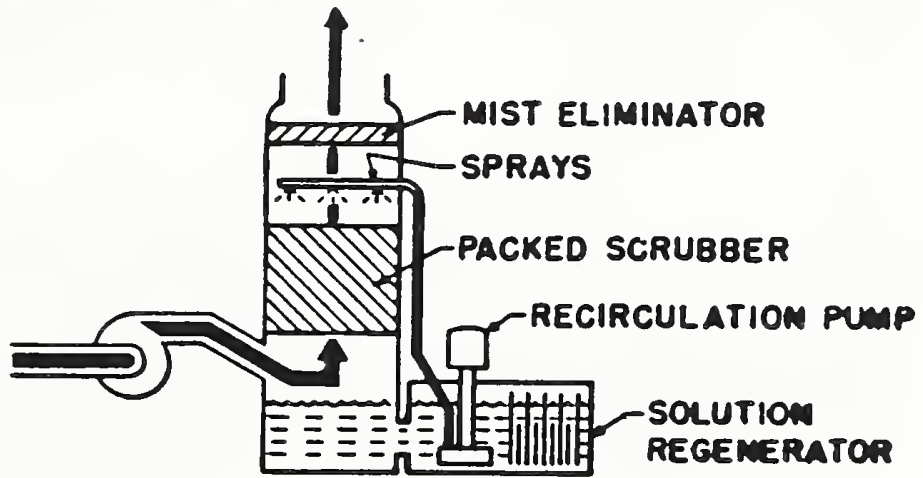

Figure 3 


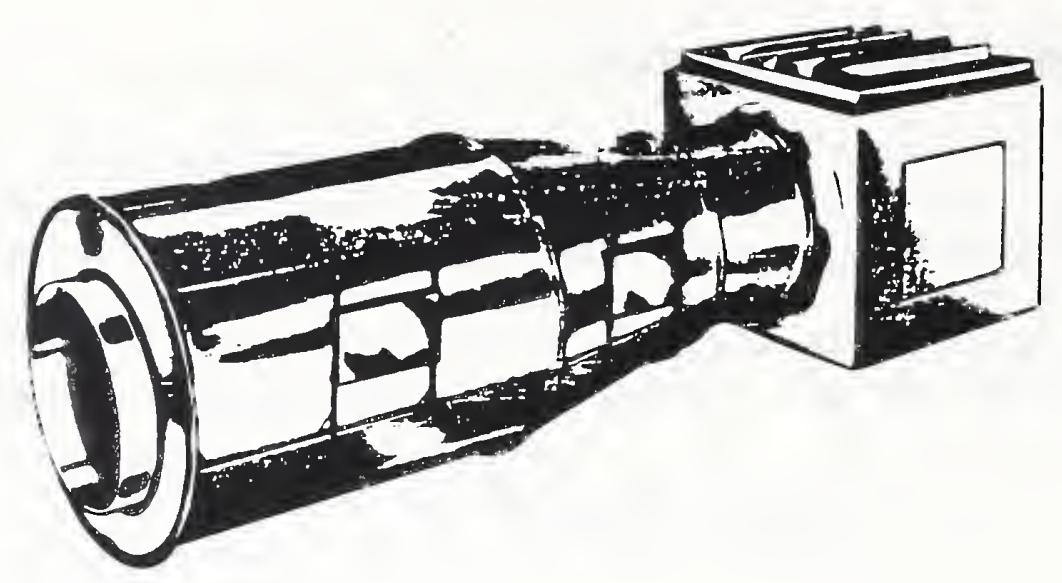

Figure 4

B. Figure 5 shows a "re-cycle loop" test. With the air flowing through the fan and back through the gas remover, a pollutant is injected, and immediately the pollutant concentration is measured, (without removing a large sample) at very frequent intervals. The results can be plotted as a decay curve (Figure 6), efficiency may be calculated from equation 3.

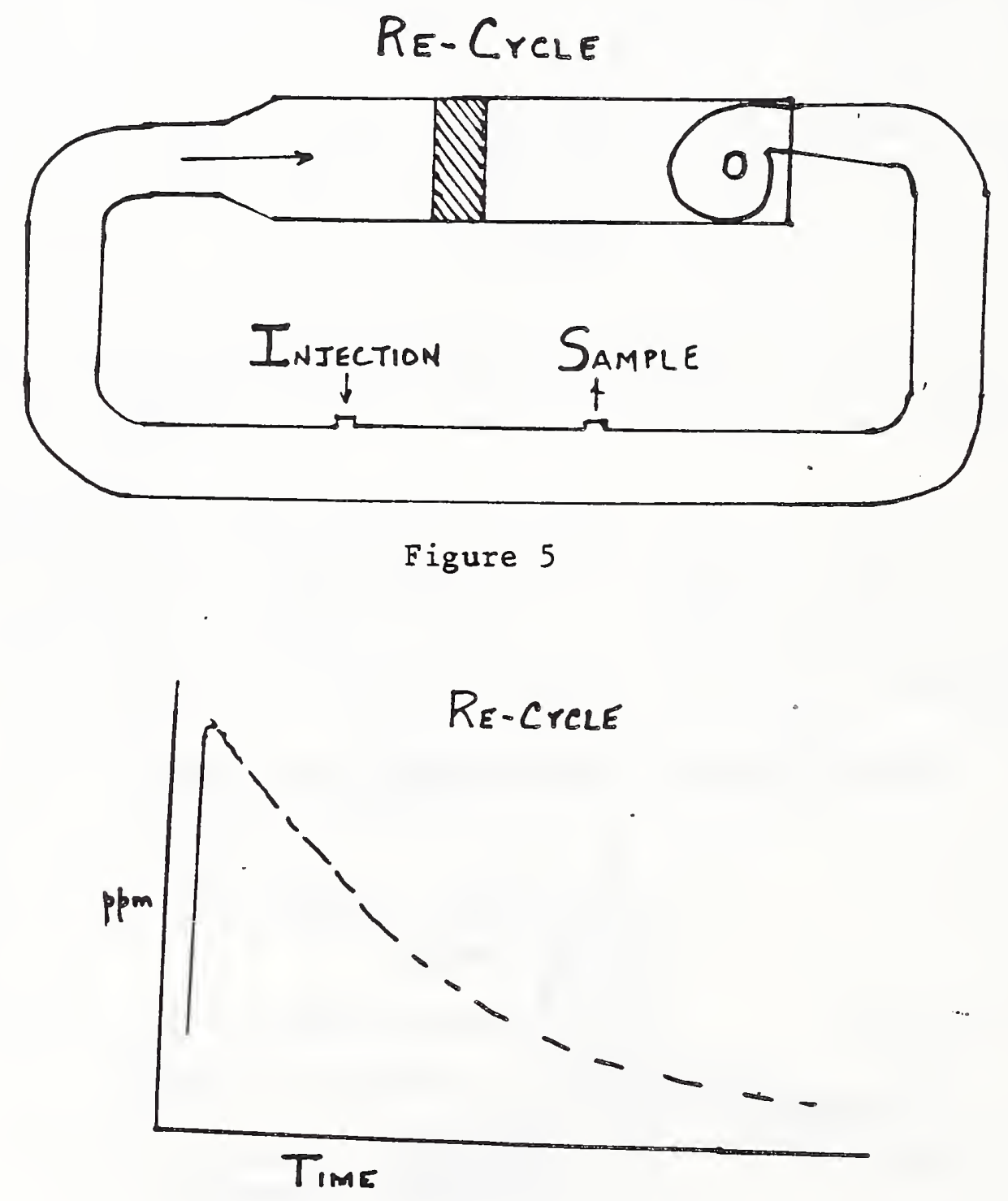

Figure 6 


$$
C=C_{0} e^{-(Q i+n Q r) T / V}+\frac{C i Q i+G}{Q e+n Q r}\left[1-e^{-(Q i+n Q r T / V)}\right]
$$

$$
C=\frac{C i Q i+G}{Q e+n Q r}
$$

$$
\mathrm{n}=\frac{\mathrm{Cu}-\mathrm{Cd}}{\mathrm{Cu}}
$$

$C=$ Concentration

$0=$ At zero time

$Q=$ Air flow

$\mathrm{n}=$ Efficiency

$\mathrm{T}=$ Time

$V=$ Volume of Space

$G=$ Rate of generation

$i=$ Infiltration

$r=$ Recirculation

$e=$ Exfiltration

$\mathrm{u}=$ Upstream

$\mathrm{d}=$ downstream

Reference 5 gives the procedure.

Pressure drop could also be measured by putting taps upstream and downstream of the filter.

C. The most practical test is an actual use test. We could use the "oncethrough" test on the scrubbers because the gas concentrations were high and easy to measure, but it could not be used in the parts per billion range. Neither could. we use the completely closed recycle test, due to instrument inaccuracy.

The chemical plant produced cellulose products by the Viscose Process. Some of the by-products of the process are sulfide gases. The gas collecting hoods over the tanks of the process are designed to collect the gases and exhaust them through scrubbers. I mention this because the order of magnitude of the concentration is so important: The range of concentration:

(a) The concentration range in the exhaust ducts going to the scrubbers is in the order of 60 to $200 \mathrm{ppm}$.

(b) The concentration leaving the scrubber might be 2 to $10 \mathrm{ppm}$. (The gas removal efficiency is $95 \%$ to $98 \%$.)

(c) Concentration in the plant might be 0 to $10 \mathrm{ppm}$, because few exhaust hoods collect $100 \%$. The OSHA 8 -hour is $20 \mathrm{pPm}$.

(d) The instrument rooms are located within the plant (see Figure 7). we hold the concentration of sulfides below $0.5 \mathrm{ppm}$ in about 12 areas to prevent corrosion of the sensitive instruments in these rooms (see Figure 8).

(e) Finally, there have been occasions when there was an upset in the process building, and the wind was in the right direction, and the sulfides were carried to the inlet of the air conditioners of the office building. Even though the concentration might only be in the range of 10 to 20 parts per billion, we had the "sick building syndrome" and I receive the complaints. 


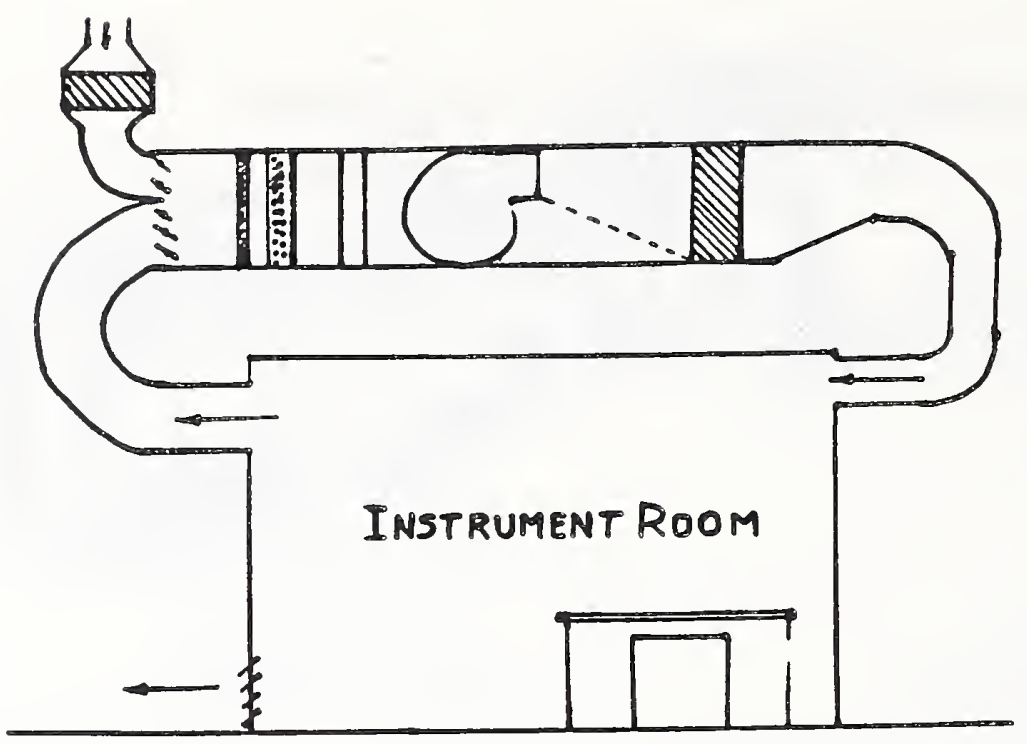

Figure 7

INSTRUMENT KOOM

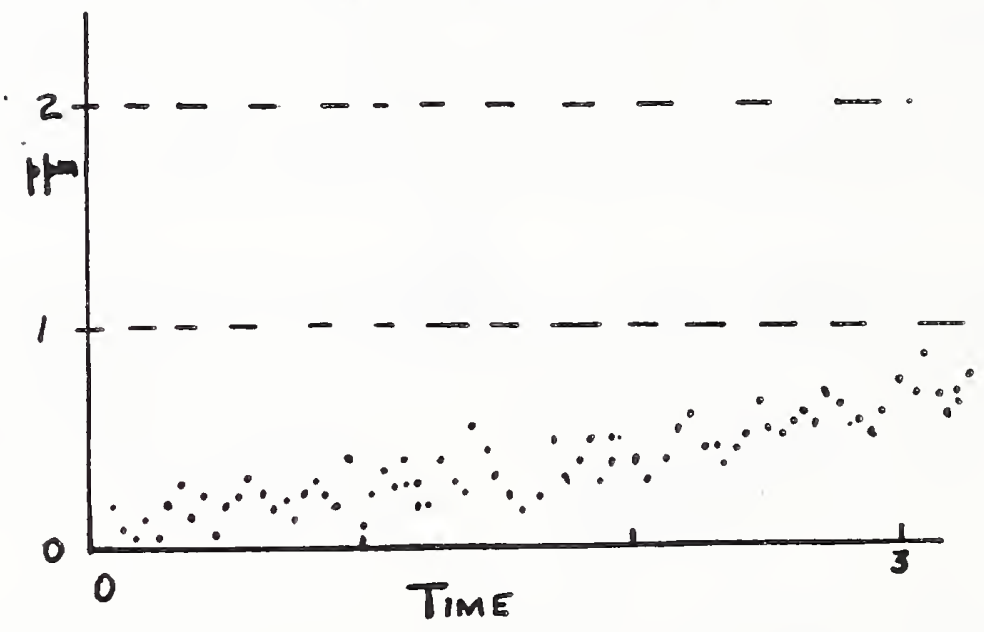

Figure 8

AN ACTUAL USE TEST:

Figure 7 shows a diagram of the equipment designed to maintain the temperature and the air quality in one instrument room, and also designed to compare gas removal filters. Outside air is brought in through a packed bed of carbon. The dampers are set after balancing the system. The outside air is mixed with some return air, and both flow through a good particulate filter and the cooling coil. (Actually thee is no heating coil here because this is a room within the heated plant, but there is a preheat coil after the carbon in the outside air duct.) The fan forces the air through the past filter, which is a replaceable bed of adsorbent.

Note that the bed is the last piece of equipment in line, in order to prevent infiltration of pollutant.

The room is slightly pressurized and the excess air flows out near the bottom through weighted dampers. When the door is opened, the dampers close, and the excess air flows out through the open door. 
The post filter was loaded first with alumina that bad been impregnated with potassium permanganate (PURAFIL) and then after three months the PURAFIL was replaced with carbon that had been impregnated with caustic. The carbon was replaced with PURAFIL after another three months, and both tests were repeated.

The low concentration of the sulfides (which is required in the instrument rooms to prevent corrosion) is difficult to measure. For these tests a lead acetate tape instrument was used. Time for recording the integrated sample was two hours, or exactly twelve continuous samples per 24 hours.

When three months of tests are plotted, the results looked something like Figure 8. The considerable scatter of the concentration was probably caused changed in concentration outside the room and the number of times the door was opened. While the temperature in the room was held at $21+$ or -2 degrees $C$, the humidity was not held constant and may have modified the effectiveness of the adsorbents.

Both the impregnated carbon and the impregnated alumina prevented the sulfide concentration from exceeding $1 / 2 \mathrm{ppm}$ in the period of three months before changing. We learned from this test that either material can be used when operating at this temperature, these humidities, and at these very variable concentrations of many pollutant gases. However, the scatter in the results was too great to prove one product superior.

\section{CONCLUSIONS:}

1. Gases and vapors can be removed from air effectively with

a. Many types of solid adsorbents with specific impregnants, and

b. Many types of liquid surfaces, with specific adsorbents and liquids.

2. The gas removal system must be designed to remove more of the specific pollutant gas than the other gases in the air.

3. The gas removal system must be evaluated at conditions similar to those expected in the application of the equipment; i.e., at the same temperature and humidity, and at the expected concentration of the pollutant and of the other components in the air. 


\section{REFERENCES :}

1. Trebal, Robert E. 1985, Mass Transfer Operations, McGraw Bill Book Co., New York, NY.

2. ASHRAE Handbook, Chapter 2, Fundamenta 18 1985, American Society of Heating, Refrigerating and Air Conditioning Engineers, Atlanta, GA.

3. Bosworth, C. M. and Barduhn, A. J., Recent Advances in Odor Control by Air Washing, Anals of the New York Academy of Sciences, Vol. 116, Art 2. P. 638 .

4. Cserme1y, T. J. and Bosworth, C.M., New Techniques for Evaluating Odor Control Methods, ASBRAE Transactions, Vo1. 70, 1964, p. 354. 
COMPARISON OF CHROMOTROPIC ACID, PARAROSANILINE,

AND GRAVIMETRIC FORMALDEHYDE DETERMINATIONS

Samuel Silberstein, Biologist

Indoor Air Quality and Ventilation Group

Building Environment Division

Center for Building Technology

National Bureau of Standards

\section{Abstract}

An automated formaldehyde emission rate monitor was developed at the National Bureau of Standards to measure emission rates of pressed wood products. A concentration monitor measured the airborne formaldehyde concentration by means of the pararosaniline method, within a medium-size dynamic measuring chamber containing a single pressed wood board. A gas chromatograph-electron capture detector measured the air exchange rate by means of the sulfur hexafluoride tracer decay method. To calibrate the formaldehyde concentration monitor, "span gas" containing a fixed formaldehyde concentration was generated by heating polyoxymethylene permeation tubes. The concentration was determined from the rate of mass loss of the tubes. Concentrations determined by the pararosaniline and gravimetric methods were compared to concentrations determined by the chromotropic acid method because the chromotropic acid method is widely used by the pressed wood industry to measure formaldehyde emission rates of their products.

It was found that formaldehyde concentrations of span gas agreed with those determined by the chromotropic acid method, validating the calibration method. Formaldehyde concentrations due to emissions from three lots of particleboard underlayment from two different manufacturers, determined by the chromotropic acid method agreed with those determined by the pararosaniline method.

Key words: Formaldehyde; indoor air quality; pressed-wood products; pararosaniline; permeation tubes; chromotropic acid.

\section{Introduction}

The pararosaniline and chromotropic acid methods, among other techniques, are widely used to measure airborne formaldehyde concentrations. For example, the pressed wood industry commonly uses the chromotropic acid method for testing the formaldehyde emissions of its products [1]. Formaldehyde emission rates are measured by placing pressed wood products into measuring chambers whose air exchange rates can be adjusted to obtain varying airborne formaldehyde concentrations. Emission rates can then be calculated from concentration and air exchange rate. Oak Ridge National Laboratory, Lawrence Berkeley Laboratory, and the National Bureau of Standards (NBS), on the other hand, commonly use the pararosaniline method [2-4] in surveys of formaldehyde concentrations and tests of emission rates of pressed wood products. The main advantage of the pararosaniline over the chromotropic acid method is that it can be automated. It would be difficult to automate the chromotropic acid method because of its requirement for concentrated sulfuric acid as a reagent, and for boiling water baths [5]. 
As part of a project for the Consumer Product Safety Commission described previously [2], NBS designed an automated pararosaniline-based system to measure formaldehyde emissions from pressed wood products. The measurements are done in medium-sized dynamic measuring chambers located in a temperature- and RH-controlled environmental chamber. The measuring chambers are tefion-lined to minimize formaldehyde absorption, and can accommodate intact $1.2-\mathrm{m} \times 2.4-\mathrm{m}$ (4-ft. $\mathrm{x} \quad 8-\mathrm{ft}$.$) boards as used in$ buildings.

NBS compared formaldehyde concentrations resulting from particleboard underlayment determined by the pararosaniline and chromotropic acid methods. Preliminary results indicate that the two methods yielded similar concentrations for the emissions of four different specimens of particleboard. The boards were from three different lots from two different manufacturers. The two methods were also shown to agree with each other on "pure" formaldehyde, by tests on standard formaldehyde atmospheres formed by emissions from polyoxymethylene permeation tubes. The concentrations of the standard atmospheres were determined by measuring the rate of mass loss of the permeation tubes.

\section{Methods}

\section{2. a. Automated Pararosaniline and Gravimetric Analyses}

A HCHO surface emission-rate measurement system was constructed by linking an airborne HCHO concentration monitor (a TGM-555 air monitor fitted with with a HCHO analytical module) to a microcomputer-based automated tracer-gas decay system used to measure air exchange rate, as described elsewhere [2]. The HCHO concentration monitor measures HCHO concentration by a modified pararosaniline method [4, 6-8]. The concentrations determined by the pararosaniline method are denoted "pa-concentrations."

Pa-concentrations were measured automatically using the HCHO concentration monitor as follows. An air sample stream was continuously pumped into the monitor at a fixed air flow rate between about 0.5 and $1.0 \mathrm{~L} / \mathrm{min}$ and scrubbed with $0.02 \%$ pararosaniline solution in $100 \mathrm{mN} \mathrm{HCl}$. Sodium sulfite solution was then added, resulting in a pararosaniline concentration of $0.013 \%$ in $67 \mathrm{mN} \mathrm{HCl}$, and a $\mathrm{Na}_{2} \mathrm{SO}_{3}$ concentration of $0.17 \mathrm{~g} / 1$ (1.3 mM). The mixture reacted for about eight to ten minutes as it was pumped through a coil to a photometer, where its absorbance at a wave length of $570 \mathrm{~nm}$ relative to a pararosaniline-HCl blank was measured.

In order to calibrate the HCHO concentration monitor, HCHO-free "zero air" and "span gas" containing a known concentration of HCHO were used [2]. Zero air was prepared by filtering room air through a Mine Safety Appliances chemical cartridge against formaldehyde vapor. Span gas was prepared by heating a permeation tube containing polyoxymethylene, a HCHO polymer, at $80^{\circ} \mathrm{C}$ in the oven of a gas standards generator and passing a HCHO-free airstream over it. Polyoxymethylene decomposes into HCHO when heated. The polymer is sealed in teflon, which is slightly permeable to HCHO [9]. The span gas concentration was calculated by the following equation:

$$
C_{S}=\left(V_{g} / M_{\mathrm{HCHO}}\right) \cdot e / F
$$


where

$\mathrm{C}_{\mathrm{S}}=$ span gas HCHO concentration, $\mathrm{ppb}$

$\mathrm{MW}_{\mathrm{HCHO}}=$ molecular weight of $\mathrm{HCHO}, 30.03$

$\mathrm{V}_{\mathrm{g}} \stackrel{\mathrm{HCHO}}{=}$ volume occupied by $\mathrm{l} \mathrm{kg}$-mole of $\mathrm{HCHO}$ at $25^{\circ} \mathrm{C}, 24.45 \mathrm{~m}^{3}$

$e^{g}=$ emission rate of $\mathrm{HCHO}$ from permeation tube, $\mathrm{ng} / \mathrm{min}$

$\mathrm{F}=$ air flow rate through gas standards generator, $\mathrm{L} / \mathrm{min}$

Formaldehyde concentrations resulting from permeation tubes are denoted "grconcentrations."

Two automated monitors located in two different environmental chambers were used. In both environmental chambers, the temperature was kept at $23^{\circ} \mathrm{C}$ and the $\mathrm{RH}$ at $50 \%$. In the first system, the air flow rate, $F$, was determined by both a wet test meter and a gas flow meter to be $2.37 \mathrm{~L} / \mathrm{s}$. Two permeation tubes were used to obtain a high enough formaldehyde concentration. The tubes were weighed approximately monthly for over one year to determine the formaldehyde permeation rate. The rate of mass loss is shown in figure 1. The emission rate was $201 \mathrm{ng} / \mathrm{min}$ with $\mathrm{r}^{2}=0.997$ and a relative error of $2 \%$, corresponding to a span gas concentration of $69 \mathrm{ppb}$. The relative error of the concentration is the difference between the relative error of the emission rate and the relative error of the flow rate. Because the relative error of the flow rate was observe to be at most as great as that of the emission rate, the maximum relative error of the concentration is approximately twice that of the emission rate.

In the second system, $F$ was determined by a gas flow meter to be $2.43 \mathrm{~L} / \mathrm{s}$. The permeation tubes were weighed approximately monthly for over one year. The rate of mass loss is shown in figure 2. The formaldehyde emission rate was $285 \mathrm{ng} / \mathrm{min}$ with $\mathrm{r}^{2}=0.998$ with a relative error of $1.1 \%$, corresponding to a span gas concentration of $95 \mathrm{ppb}$.

\section{2. b. Chromotropic acid analysis}

The chromotropic acid analysis was performed as follows. For one hour or more, air was drawn through teflon tubing into a midget impinger or two in series ("double impinger test") containing $20 \mathrm{ml}$ of $1 \frac{\circ}{\partial} \mathrm{Na}_{2} \mathrm{HSO}_{3}$, a canister containing desiccant, and a flowmeter, which was carefully calibrated with a bubble meter, and a pump at $1 \mathrm{~L} / \mathrm{min}$. The solution was then analyzed by the chromotropic acid method, using formalin standardized by titration as a calibration standard [5]. All sampling was done in duplicate, and two analyses were done for each sample. Formaldehyde concentrations determined by the chromotropic acid method are denoted "ca-concentrations."

\section{2. c. Medium-size dynamic measuring chambers}

The medium-size dynamic measuring chambers for determining HCHO surface emission rates of individual pressed-wood products are shown schematically in figure 3 . The interior dimensions are $1,2 \mathrm{~m} \times 2.4 \mathrm{~m} \times 0.6 \mathrm{~m}(4 \mathrm{ft} . \mathrm{x} 8$ $\mathrm{ft} . \mathrm{x} 2 \mathrm{ft.})$, for a volume of $1.8 \mathrm{~m}^{3}\left(64 \mathrm{ft} .{ }^{3}\right)$. All inner exposed surfaces were lined with teflon sheets to minimize HÇHO adsorption. Two small DC fans with a rated capacity of $7 \mathrm{~L} / \mathrm{s}(15 \mathrm{ft} .3 / \mathrm{min})$ were installed at both 


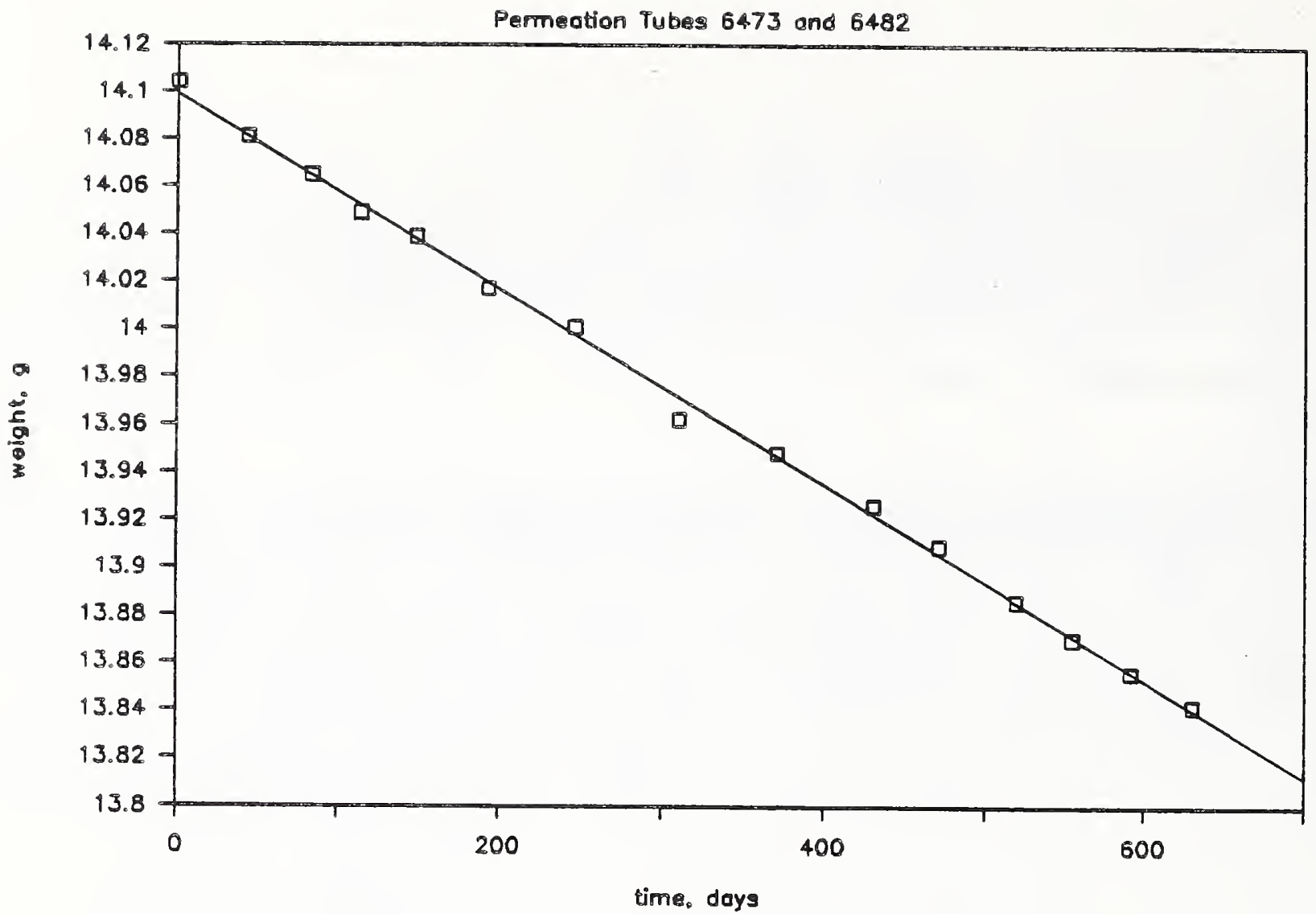

Figure 1. Mass loss calibration for permeation tubes used for calibrating HCHO monitor \#I.

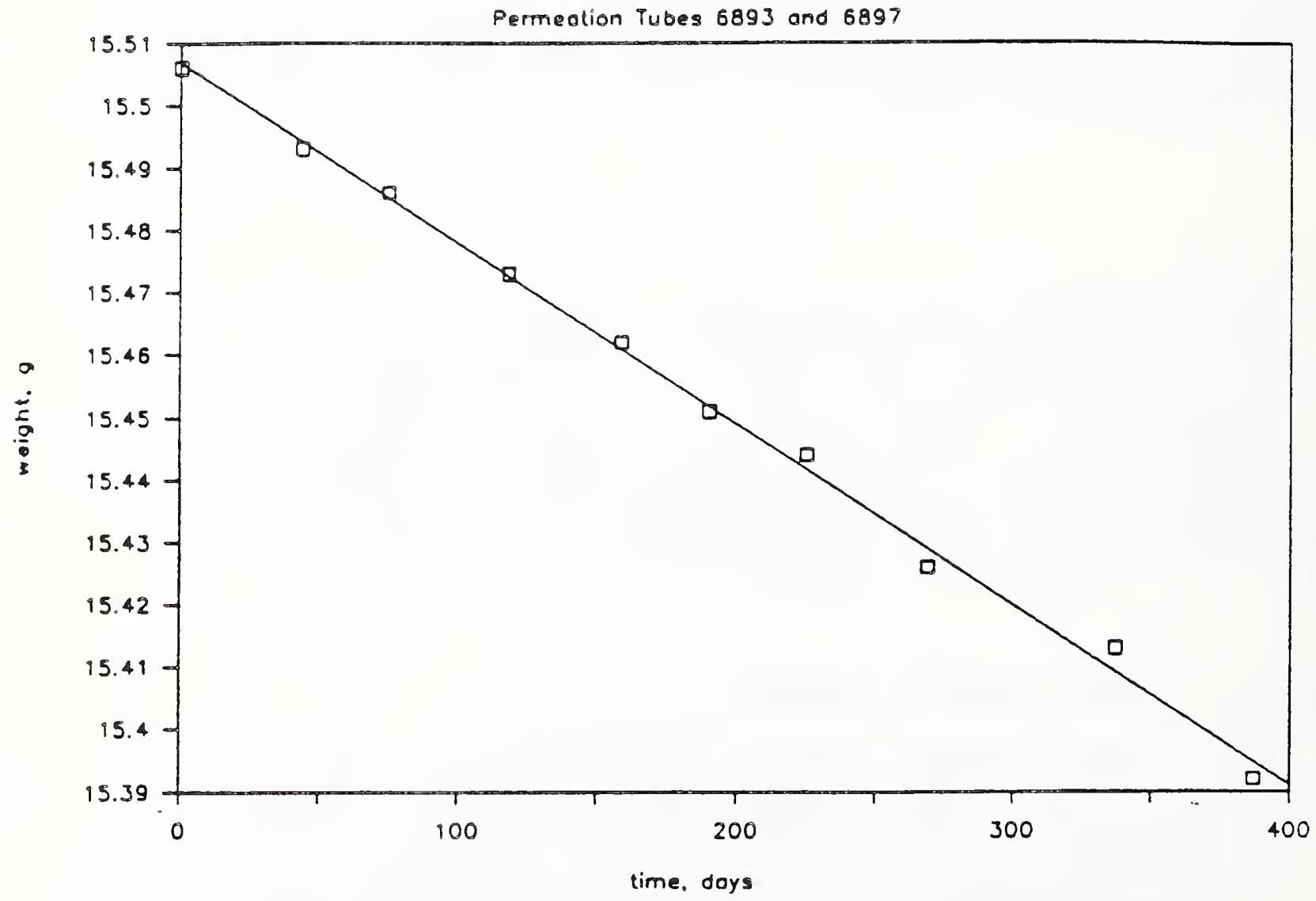

Figure 2. Mass loss calibration for permeation tubes used for making calibrating HCHO monitor \#2. 
ends of the chambers to supply and exhaust the air. Three valves in the system controlled the amount of air brought in, exhausted, and recirculated. The fans were run at constant speed and the air exchange rate was controlled by the three valves in order to maintain a constant air velocity over the sample. The outlet valve was usually adjusted to slightly pressurize the chamber, thus assuring that air entered only through the inlet. Further details of the dynamic measuring chambers are given elsewhere [2].

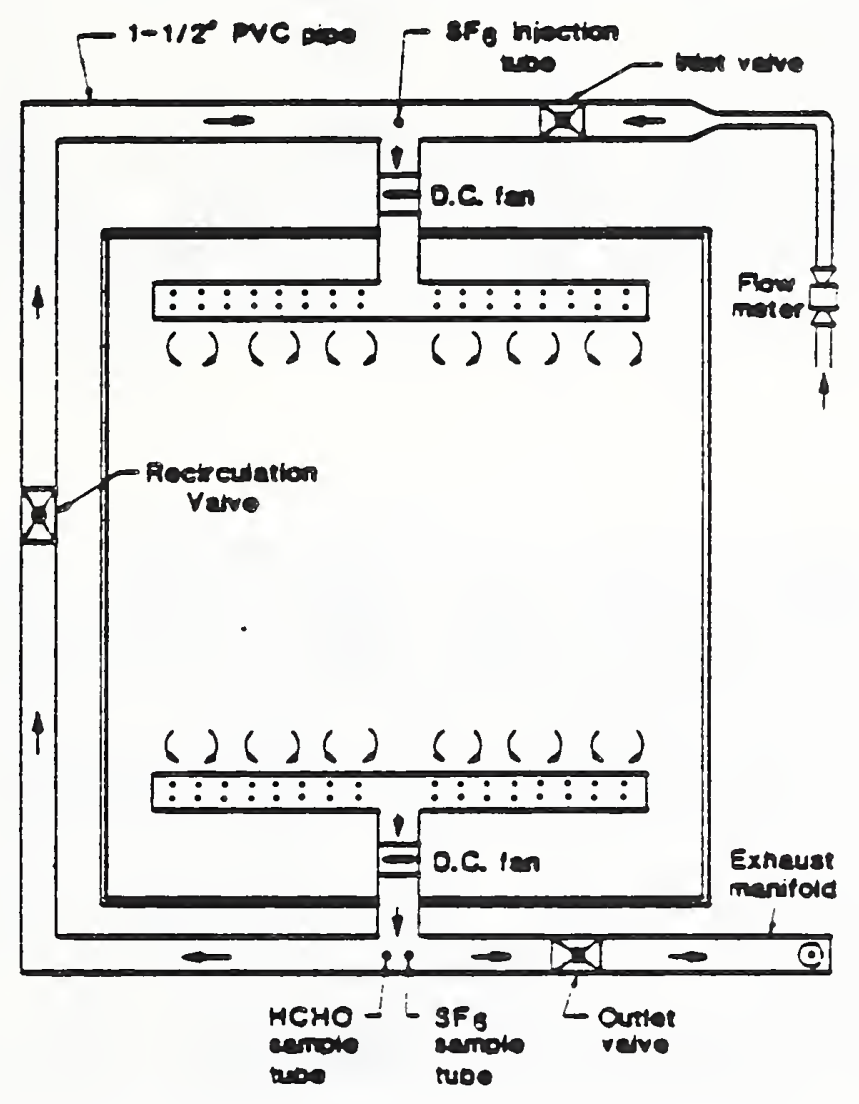

Eigure 3. Schematic of medium-size chamber for measuring HCHO emission rates.

Air exchange rates were measured using a tracer-gas decay method [2, 10]. Sulfur hexafluoride was injected into the inlet of the chamber and sampled at the outlet. The HCHO concentration was also sampled at the outlet. Good air mixing within the chambers was demonstrated through smoke visualization of the air-flow patterns within the chamber. After releasing smoke into the chamber inlet, the smoke density quickly became uniform in the chamber and there were no dead spots.

It was reported elsewhere [11] that only background levels of formaldehyde could be detected in empty measuring chambers. It was further reported that when formaldehyde from permeation tubes was injected into an empty measuring chamber, the pa-concentration agreed with the concentration predicted from the emission rate of the permeation tube and the air exchange rate.

2. d. Pressed wood products

Four different particleboard underlayment specimens were measured in mediumsize chambers located in the two different environmental chambers. The four boards are listed in table 1. Boards B2, B3, and B8, all from one 
manufacturer, were measured in the first system, and board G11, from another manufacturer, was measured in the second system. Board B2 was from one lot; boards B3 and B8 from another. Boards B3 and B8 were each measured at two different air exchange rates in order to obtain additional formaldehyde concentrations.

\section{2. e. Linear regression analysis}

Ca-concentrations were compared to pa-concentrations by a best fit linear regression line of the form ca-concentration = slope $\cdot$ pa-concentration. Similarly, ca-concentrations were compared to gr-concentrations by a best fit linear regression line of the form ca-concentration $=$ slope $\cdot g r-$ concentration.

\section{Results}

Comparisons between several mean ca-concentrations and mean paconcentrations of particleboard underlayment, and between ca-concentrations and gr-concentrations of two different span gases are shown in table 1 and figures 4-5. The formaldehyde concentrations covered a pa-concentration range of 50-400 ppb, and a gr-concentration range of 70-100 ppb. The deviation of each of the two ca-concentrations from their mean was less than $12 \%$ for concentrations less than $100 \mathrm{ppb}$, and less than $5 \%$ for concentrations greater than $100 \mathrm{ppb}$. The standard deviation of paconcentrations ranged between $8-21 \%$, with no evident dependence on concentration. As discussed in the methods section above, the relative errors in the gr-concentrations were estimated to be under $5 \%$. Double impinger tests were discontinued when no formaldehyde was found in the second impinger.

Eigure 4 shows the line of identity between ca- and pa-concentrations, and the best-fit linear regression line of the form ca-concentration = slope. pa-concentration. Table 1 shows that the regression line has a slope of 0.91 , with an error of 0.02 and $r^{2}=0.99$. Table 1 also shows ratios of cato pa-concentration. These are all between 0.89 and 1.14 . Every caconcentration except one is within 1 pa-concentration standard deviation unit of the pa-concentration. The remaining ca-concentration, $53 \mathrm{ppb}$, is within 2 standard deviation units of the pa-concentration, $46 \mathrm{ppb}$. The conclusion is that ca- and pa-concentrations of the pressed-wood products tested are nearly indistinguishable from each other.

Figure 5 shows the line of identity between ca- and gr-concentrations. The best-fit linear regression line of the form ca-concentration = slope $\cdot g r-$ concentration is not shown in the figure because it nearly coincides with the line of identity. Table 1 shows that the regression line has a slope of 0.99 , with an error of 0.07 and $r^{2}=0.82$. This means that the ca- and grconcentrations are nearly identical to each other. 
Comparison between formaldehyde concentrations determined by chromotropic acid with concentrations detemined by pararosaniline or gravimetrically

$\begin{array}{lccccrrrr}\text { board } & \text { date } & \text { CA } & \text { dif } & \text { dif/CA } & \text { PA } & \text { SD } & \text { SD/PA } & \text { CA/PA } \\ \text { B3 } & 5 / 1 / 86 & 60 & 2 & 0.03 & 61 & 6 & 0.11 & 0.99 \\ \text { B8 } & 5 / 1 / 86 & 53 & 3 & 0.05 & 46 & 6 & 0.12 & 1.14 \\ \text { B2 } & 5 / 2 / 86 & 116 & 4 & 0.04 & 127 & 27 & 0.21 & 0.91 \\ \text { B3 } & 5 / 2 / 86 & 123 & 5 & 0.04 & 124 & 25 & 0.20 & 0.99 \\ \text { B8 } & 5 / 2 / 86 & 122 & 3 & 0.02 & 126 & 25 & 0.20 & 0.97 \\ \text { G11 } & 5 / 7 / 86 & 366 & 5 & 0.01 & 410 & 64 & 0.16 & 0.89\end{array}$

slope of linear regression line of $\mathrm{CA}=$ slope * $\mathrm{PA} \quad 0.91$

standard error of slope 0.02

$\begin{array}{ll}r^{2} & 0.99\end{array}$

$\begin{array}{llrrcl}\text { GR } & \text { date } & \text { CA } & \text { dif } & \text { dif } / C A^{*} & \text { CA/GR } \\ 69 & 5 / 1 / 86 & 71 & 8 & 0.11 & 1.03 \\ 95 & 5 / 16 / 86 & 101 & 0 & 0.00 & 1.05 \\ 69 & 5 / 30 / 86 & 57 & 4 & 0.07 & 0.83\end{array}$

slope of linear regression line of $\mathrm{CA}=$ slope * $\mathrm{GR} \quad 0.99$

standard error of slope $\quad 0.07$

$\begin{array}{ll}r^{2} & 0.82\end{array}$

$\mathrm{CA}=$ average of 2 formaldehyde concentrations determined by chromotropic acid, ppb

dif = absolute value of the difference between either formaldehyde concentration determined by chromotropic acid and $\mathrm{CA}$, $\mathrm{ppb}$

$\mathrm{PA}=$ average formaldehyde concentration determined by pararosaniline, ppb

$S D=$ standard deviation of $P R, p p b$

$A E R$ = air exchange rate, $\mathrm{h}^{-1}$

$\mathrm{GR}=$ span gas concentration determined gravimetrically, ppb

*The two quantities in this expression were not rounded before division.

Discussion

Agreement between mean ca- and gr-concentrations, their small errors, and the inability to detect higher than background concentrations in the second impinger of a double impinger test, show that formaldehyde collection efficiency by the first impinger was nearly $100 \%$. Since the automated procedure is based on permeation tubes, the results of the experiment reported here suggest that the pararosaniline and chromotropic acid methods would agree on "pure" formaldehyde atmospheres produced by heating polyoxymethylene permeation tubes. Preliminary experiments further suggest that pa- and ca-concentrations agree with each other on the pressed-wood products tested. Further tests are needed to expand these results to a wider variety of pressed-wood products from different manufacturers.

One question of interest is whether processes used in the manufacture of pressed-wood products may result in the emission of substances that are 


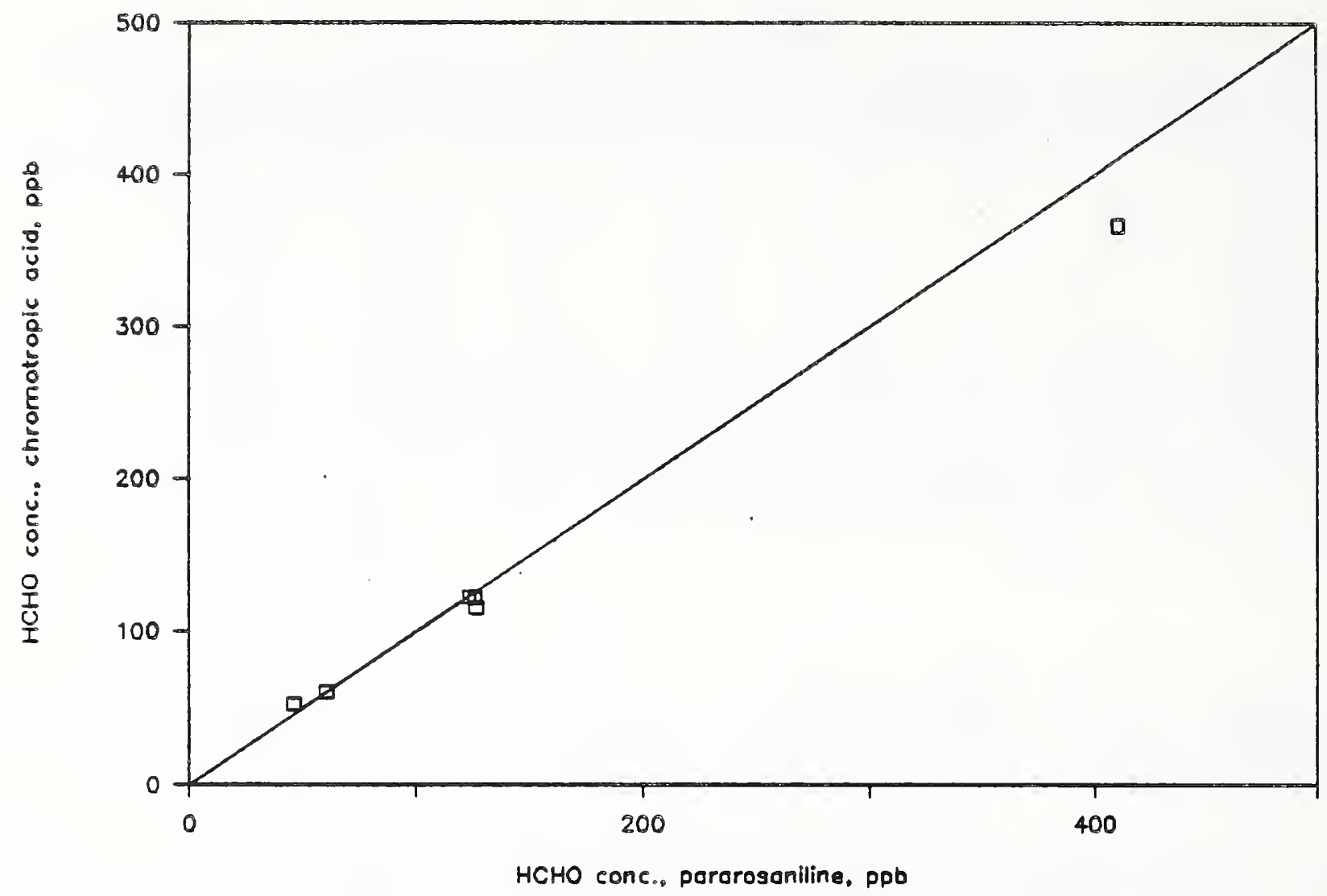

Figure 4. Ca-vs. pa-concentrations of particleboard. The line is the line of identity.

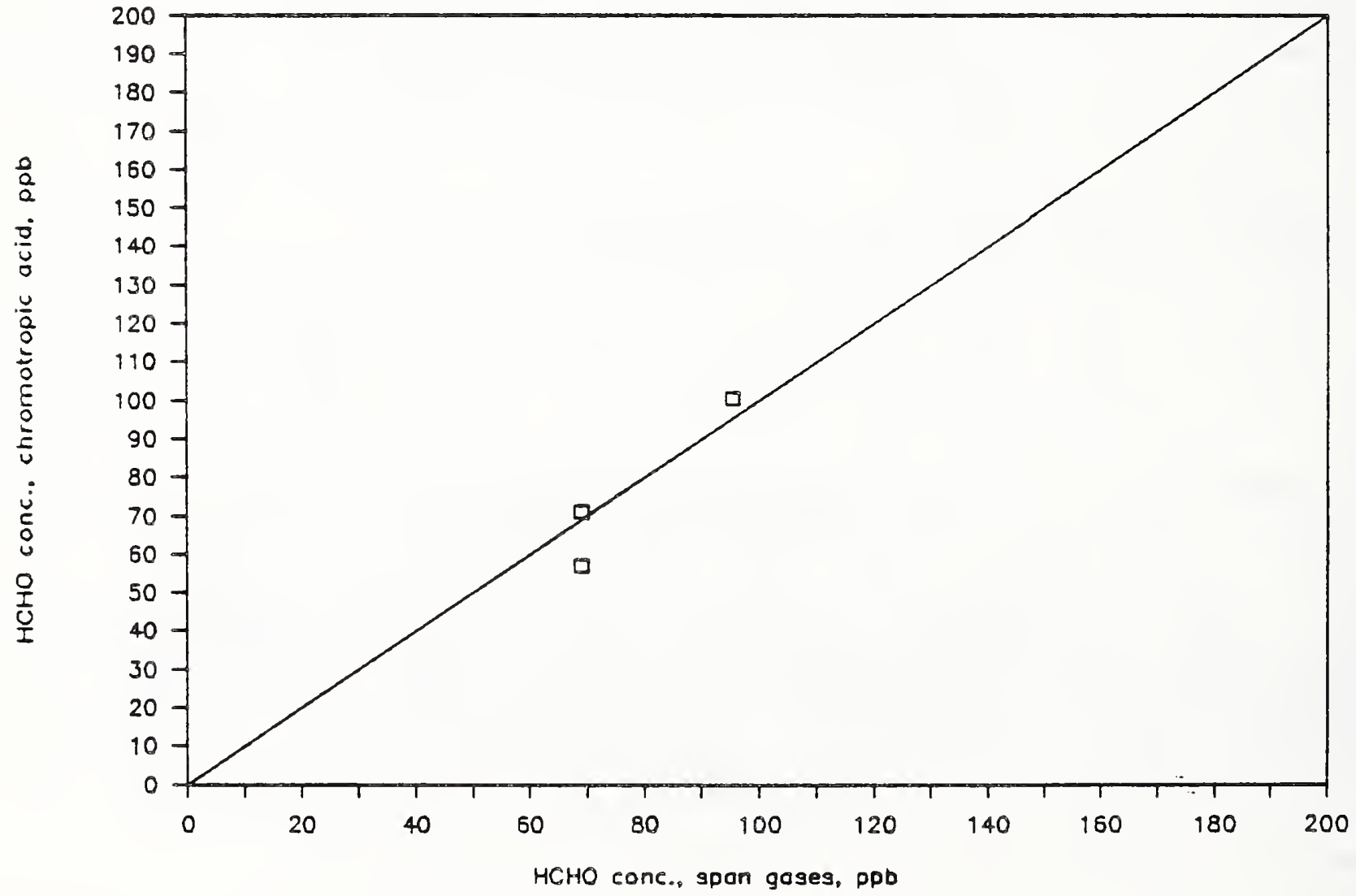

Eigure 5. Ca-vs. gr-concentrations of span gases. The line is the line of identity. 
interferences for the pararosaniline or chromotropic acid methods. Higher molecular weight aldehydes give positive interference for both methods, but only if their concentrations are far in excess of formaldehyde concentration, which is most unlikely [4-5]. Phenol, ethanol, higher molecular weight alcohols, olefins, and sulfur dioxide give negative interferences for the chromotropic acid method [5, 12]. It is possible that some of these compounds may be emitted by underlayment. The present study shows that any interfering substances emitted by the boards did not differentially affect the two analytical methods.

\section{Acknowledgment}

This project was made possible by support from the U. S. Consumer Product Safety Commission.

\section{References}

[1] Versar, Inc., "Formaldehyde Exposure in residential settings: sources, levels, and effectiveness of control options," prepared for $U$. S. Environmental Protection Agency, Springfield, VA, 1986.

[2] R. A. Grot, S. Silberstein, and K. Ishiguro, "Validation of Models for Predicting Formaldehyde Concentrations in Residences due to Pressed Wood Products -- Phase I," National Bureau of Standards Interagency Report No. 85-3255, Gaithersburg, MD, 1985.

[3] T. G. Matthews, T. J. Reed, B. J. Tromberg, K. W. Fung, C. V. Thompson; J. O. Simpson, and A. R. Hawthorne, "Modeling and Testing of Formaldehyde Emission .Characteristics of Pressed-Wood Products," Report no. 18 to CPSC, Oak Ridge National Laboratory Report no. TM $9867,1985$.

[4] R. R. Miksch et al., "Modified Pararosaniline Method for the Determination of Formaldehyde in Air," Anal. Chem. 53, 2118-2123, 1981.

[5] National Institute for Occupational Safety and Health, Manual of Analytical Methods, 3rd ed.; P. M. Eller, Ed., NIOSH Publication No. 84-100, Method 3500, Cincinnati, 1984.

[6] National Research Council, "Formaldehyde and Other Aldehydes," National Academy Press, 1981, Washington.

[7] G. R. Lyles, F. B. Dowling and V. J. Blanchard, "Quantitative Determination of Formaldehyde in Parts Per Hundred Million Concentration Level," J. Air Pollut. Control Assoc. 15, 106-108, 1965.

[8] CEA Instruments, Inc., "Low Level Formaldehyde in Air (Mercury-Eree Procedures)," Emerson, NJ, 1984. 
[9] F. P. Scaringelli, A. E. O'Keefe, E. Rosenberg and J. P. Bell, "Preparation of known Concentrations of Gases and Vapors with Permeation Devices Calibrated Gravimetrically," Anal. Chem. 42, 871876, 1970.

[10] R. A. Grot, D. M. Burch, S. Silberstein, and L. Galowin, "Measurement Methods for Evaluation of Thermal Integrity of Building Envelopes," National Bureau of Standards Interagency Report 82-2605, Gaithersburg, MD, 1982 .

[11] S. Silberstein, R. A. Grot, K. Ishiguro, and W. S. Dols, "Validarion of Models for Predicting Formaldehyde Concentrations in Residences due to Pressed Wood Products -- Final Report," in preparation.

[12] E. R. Kennedy, A. W. Teass, and Y. T. Gagnon, "Industrial Hygiene Sampling and Analytical Methods for Formaldehyde: Past and Present," pp. 3-12 in V. Turoski, ed., "Formaldehyde," Advances in Chemistry Series 210, American Chemical Society, Washington, 1985. 
Discussion and Conclusions - Preston E. McNall, NBS

After the prepared presentations, an open discussion was held for one and one-half hours, led by the editor. The purpose was to summarize what was known about test methods, and to outline future directions. The editor presents his impressions here. It is impossible to attribute statements here to the individuals who took part in the discussion.

Challenge materials. It was agreed that a number of challenge materials should be used to represent the wide range of materials expected to be encountered in practice. It was felt that seven to ten different materials might suffice, for example:

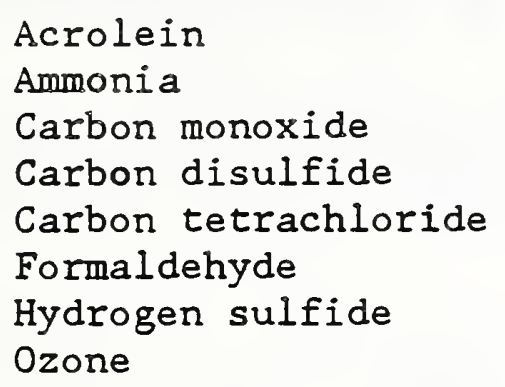

These materials, except ozone and formaldehyde, could be prepared in various concentrations in cylinders, and form a standard "cocktail" to be supplied to candidate devices. More work is needed to determine the challenge materials to be used, but this appeared to be the most practical approach.

Other contaminants could be added by the user, if such contaminants were handled similarly, as appropriate to the individual equipment in application. The user would need to show the importance of the added contaminants.

Challenge Concentrations. Because the performance of most commonly used devices is affected drastically by concentration, the challenge concentrations must be kept close to those expected to be encountered in the field. High concentrations could cause non-representative results.

Test time and cost problems for loading tests were recognized.

Ambient Test Conditions. Obviously, test conditions should approach those encountered in practice. It was noted that humidity, as well as temperature, often affect performance significantly. Since most equipment will be installed in return air ducts, and since high humidities are usually detrimental to performance, it was agreed that conditions of about $\left(21^{\circ} \mathrm{C}\right),\left(70^{\circ} \mathrm{F}\right)$ and $50 \% \mathrm{RH}$ be used. Options might be considered for including other conditions for special equipment, but much of the time $70^{\circ} \mathrm{F}$ and $50 \% \mathrm{RH}$ will be appropriate. 
Equipment Type. Several types of equipment may be used. Adsorption, absorption, chemisorption, catalysis and electric fields are known methods. While these operate differently on contaminants, it was concluded that all should be evaluated similarly. Special considerations, involving breakthrough, selective off-gassing of previously retained materials, and penetration or off-gassing of chemically-changed materials could be included in tests for different types of equipment if appropriate. However, similar challenge materials seemed to be most representative of field conditions, and should be used for all types of filters.

Types of Tests. Two general types of tests are necessary. First, a test of the performance of a new or regenerated filter should be performed. For this purpose, the efficiencies (or penetrations) of the new equipment should be reported on each of the challenge materials. These should be measured at test conditions reasonably expected in the field, as described elsewhere. Second, the performance life, or loading must be established under reasonable conditions representative of those to be encountered in the field:

\section{Detailed Test Procedures}

General. Filter evaluations have generally been made in two ways. First, a test duct has been employed, in which the equipment can be mounted. Measurements are made on the filters on a single-pass mode. Efficiencies, etc. are then single-pass for the filter. Second, a roomsize, or smaller chamber has been used, and the filters placed in a recirculating airflow arrangement. Concentrations are measured in the chamber. It was decided to employ the single-pass method, because the filter field application requires the single-pass parameters to predict field performance. In the chamber tests, the single-pass efficiency must be calculated from the chamber system measurements. Another important factor was that the ASHRAE Standard 52 for particulate filter evaluation employs a test duct, which could probably be used for gaseous and vaporous evaluation as well.

In conclusion, a test duct similar to that of ASHRAE Standard 52 seemed to be the most appropriate choice.

Contaminant Feeding Methods. A "cocktail" of contaminants as described earlier could be prepared and packaged in cylinders of various sizes. The entrance section of the test duct should be equipped with suitable prefilters to condition the outside, or laboratory air. Heaters, coolers and humidity contol would also be necessary. The conbtaminants would be introduced from the cylinders at a controlled rate to provide the desired upstream concentrations. Mixing baffles would probably be required. Nonstorable contaminants, such as ozone, would require different feeding systems as appropriate. Formaldehyde would have to be kept separate from ozone and $\mathrm{NH}_{3}$.

Measuring Means. Upstream and downstream concentrations need to be 
measured so that single-pass efficiencies (or penetrations) can be evaluated directly. Gas chromatography seemed to be the most appropriate choice. Other methods for specific contaminants may also be required.

Weighing of filters to obtain retention values would not usually be appropriate due to spurious retention of moisture and other unknown contaminants.

"Clean" Efficiency Tests. "As received" filters need efficiency evaluations as important parameters. Approximately $2^{\prime} \times 2^{\prime}$ sample filters representative of field models are needed (consistent with ASHRAE Standard 52). Evaluations would be reported on the cocktail contaminants separately for each. As previously mentioned, concentrations would be low and representative of field use.

The face velocity would be representative of field use, about $500 \mathrm{ft} / \mathrm{min}$ corresponding to about $2000 \mathrm{cfm}$. Higher and lower velocities may also be appropriate to cover ranges of field use. "Clean" pressure drop results are needed for application and economics.

Loading Tests. An appropriate loading test is needed to determine filter life. To save challenge gases and time it was decided, for practical reasons, to feed concentrations at perhaps 10 times the "clean" test rate, to representative smaller filter samples. A $3 " x 3^{\prime \prime}$ section or even smaller was thought appropriate. Of course, practical filter sections may not lend themselves to sections this small, and larger sections for those filters would be necessary. If this procedure worked, a test of 100-200 hours might be appropriate.

Pressure drop vs. loading information would also be obtained several times throughout the test. Breakthrough information on these challenge contaminants would be measured and reported several times also.

There was much discussion of when to terminate such a loading test. For general ventilation purposes, clean efficiencies of $90 \%$ or so would be particularly useful (penetration 10\%). Perhaps the test could be terminated at a doubling of "clean" penetration of $1 / 2$ the number challenge materials. More research and consensus are necessary. Chemisorption filters would also have to be monitored for off-gassing of other unwanted materials.

\section{Conclusions}

In application the designer needs to know answers to four important questions:

1. What does the "clean" filter do?

2. How long does it last?

3. What penalty, energy, etc. is associated with its pressure drop? 
4. What are its first cost, regeneration or replacement costs, and product life?

With this information the designer can estimate, for field application, the value (performance) of the filter and its life-cycle cost. The test procedures should answer the first three questions and the manufacturer must establish the answer to question 4.

The workshop was successful in helping to describe the state of the art, and to set a framework for future test development and research. Although all of the appropriate experts could not participate, it is hoped that this document will stimulate more public presentations of important research results. 
1. PUBLICATION OR REPORT NO.

NBSIR $88-3716$
2. Performing Organ. Report Nod 3. Publication Date

MARCH 1988

TITLE AND SUBTITLE

Proceedings of the Symposium on Gaseous and Vaporous

Removal Equipment Test Methods

AUTHOR(S)

P. E. McNall

PERFORMING ORGANIZATION (If joint or other than NBS, see instructions)

7. Contract/Grant No.

NATIONAL BUREAU OF STANDARDS

U.S. DEPARTMENT OF COMMERCE

8. Type of Report \& Period Covered

GAITHERSBURG, MD 20899

SPONSORING ORGANIZATION NAME AND COMPLETE ADDRESS (Street, City, State, ZIP)

NBS

- SUPPLEMENTARY NOTES

Document describes a computer program: SF-185, FIPS Software Summary, is attached.

- ABSTRACT (A 200-word or less factual summary of most significant information. If document includes a significant bibliography or literature survey, mention it here)

his symposium proceedings summarizes the current state-of-the-art on gaseous and aporous removal test methods for equipment designed for use in the general ventilation f buildings. Papers by the ten (10) invited authors are included. A discussion ection outlines the conclusions reached concerning the future direction of test lethod development.

$\therefore$ KEY WORDS (Six to twelve entries; alphabetical order; capitalize only proper names; and separate key words by semicolons) absorption; adsorption; air cleaning; catalysis, chemisorption; gaseous; validation; vapors

i. AVAILABILITY

Unlimited

For Official Distribution. Do Not Release to NTIS

Order From Superintendent of Documents, U.S. Government Printing Office, Washington, D.C. 20402.

X] Order From National Technical Information Service (NTIS), Springfield, VA. 2216।
14. NO. OF PRINTED PAGES

90

15. Price

$\$ 13.95$ 



$$
\text { . }
$$


